\title{
Quantum molecular master equations
}

\author{
Sylvain D. Brechet, ${ }^{*}$ Francois A. Reuse, Klaus Maschke, and Jean-Philippe Ansermet \\ Institute of Condensed Matter Physics, Station 3, Ecole Polytechnique Fédérale de Lausanne-EPFL, CH-1015 Lausanne, Switzerland
}

(Received 2 July 2016; published 5 October 2016)

\begin{abstract}
We present the quantum master equations for midsize molecules in the presence of an external magnetic field. The Hamiltonian describing the dynamics of a molecule accounts for the molecular deformation and orientation properties, as well as for the electronic properties. In order to establish the master equations governing the relaxation of free-standing molecules, we have to split the molecule into two weakly interacting parts, a bath and a bathed system. The adequate choice of these systems depends on the specific physical system under consideration. Here we consider a first system consisting of the molecular deformation and orientation properties and the electronic spin properties and a second system composed of the remaining electronic spatial properties. If the characteristic time scale associated with the second system is small with respect to that of the first, the second may be considered as a bath for the first. Assuming that both systems are weakly coupled and initially weakly correlated, we obtain the corresponding master equations. They describe notably the relaxation of magnetic properties of midsize molecules, where the change of the statistical properties of the electronic orbitals is expected to be slow with respect to the evolution time scale of the bathed system.
\end{abstract}

DOI: 10.1103/PhysRevA.94.042505

\section{INTRODUCTION}

Relaxation of molecular magnetic moments has been observed in ferromagnetic clusters. De Heer et al. [1-3] and Chatelain [4] investigated the deflection of iron clusters in a Stern-Gerlach experiment, and Payne et al. [5] performed a similar experiment for cobalt clusters. In contrast to the normal deflection of single atoms [6-8], an anomalous one-sided deflection is observed in the field gradient direction, which can be attributed to spin relaxation processes within the cluster $[9,10]$.

The theoretical description of nonradiative molecular relaxation processes is a challenging problem. Several attempts to explain the relaxation of molecular magnetic moments in terms of resonant and phonon-assisted quantum tunneling can be found in the literature $[11,12]$. Such purely phenomenological approaches are, however, not satisfactory since they provide no insight into the relaxation mechanism. A better understanding can be attained within a statistical approach.

Here we adopt a quantum description of an open system [13] that interacts with a bath [14]. The evolution of the system is governed by quantum master equations. These equations determine the evolution of the density operator satisfying the von Neumann conditions [15] and allowing for a statistical description of the system [16]. Bloch, Wangsness, and Redfield [17-19] developed the theoretical framework of the master equations to describe relaxation. The general form for the Markovian master equations was established by Lindblad [20]. The Markovian master equations were used to study vibrational relaxation processes in condensed media by Lin [21] and Jean et al. [22], in molecules by Tung and Yuan [23], May and Schreiber [24], Gao [25], and Linden May [26].

We consider a system weakly coupled and weakly correlated to a bath at statistical equilibrium [27]. The distinction between the bath and the system is essentially determined

*Corresponding author: sylvain.brechet@epfl.ch by the respective characteristic time scales [13,14]. The time scale characterizing the dynamics of the bath must be much smaller than the time scale characterizing the dynamics of the system $[27,28]$. These characteristic time scales depend on the nature and the size of the system and of the bath.

We extend the formalism developed in [29] and apply it to a free-standing molecule. The states involved in the evolution of a molecule belong to a subspace of the Hilbert space restricted to the neighborhood of the molecular ground state. In principle, the choice of system and bath could depend on the particular molecule under consideration. Here we attribute the molecular deformation and orientation properties, as well as the electronic spin properties to the system and the electronic spatial properties to the bath. We expect this choice to be adequate for the description of the relaxation of the net molecular magnetic moment in a midsize molecule.

The explicit expression of the molecular Hamiltonian used in our analysis is based on our recent work [30], where the molecular orientation is treated as a genuine quantum property. In that article, the Hamiltonian of the system is expressed in terms of observables describing global and internal physical properties of the system. In a classical framework, these observables would refer to a rotating frame attached to the molecule. However, this is impossible in a quantum framework, since the positions of the nuclei, and thus the orientation of the molecule, are described by operators. The molecular orientation operator satisfies rotational canonical commutation relations with the molecular orbital angular momentum operator [30]. The dynamical contributions with the spin properties of the nuclei can be neglected [31].

This article is organized in the following way. The description of a molecule is established in Sec. II. The mathematical structure of the general master equations is detailed in Sec. III. The master equations are specified for a system having molecular deformation and orientation properties as well as electronic spin properties, interacting with a bath having electronic spatial properties and with an external magnetic field in Sec. IV. Finally, explicit expressions for the transition rates are given in Sec. V. 


\section{DESCRIPTION OF A MOLECULE}

\section{A. Hilbert spaces}

We consider a molecule composed of $N$ nuclei and $n$ electrons. The properties of the $N$ nuclei without spin are described by the closed subspaces of the Hilbert space $\mathcal{H}_{N}$. The properties of the $n$ electrons are described by the closed subspaces of the Hilbert space $\mathcal{P}^{\left[1^{n}\right]} \mathcal{H}_{e}^{\otimes n}$, where the Hilbert space $\mathcal{H}_{e}$ describes the properties of a single electron and the orthogonal projector $\mathcal{P}^{\left[1^{n}\right]}$, which projects the Hilbert space $\mathcal{H}_{e}^{\otimes n}$ on the subspace of totally antisymmetric tensors of $\mathcal{H}_{e}^{\otimes n}$, shall be expressed explicitly below. In this description, the electrons are treated as fermions. The nature of the nuclei does not need to be taken explicitly into account because the overlap integrals are negligible. In order to build the Hilbert space describing the properties of the molecule, we introduce first the Hilbert space $\mathcal{H}$, defined as

$$
\mathcal{H}=\mathcal{H}_{N} \otimes \mathcal{H}_{e}^{\otimes n}
$$

The space of linear operators acting on the Hilbert space (1) is expressed as

$$
\mathcal{L}(\mathcal{H})=\mathcal{L}\left(\mathcal{H}_{N}\right) \otimes \mathcal{L}\left(\mathcal{H}_{e}^{\otimes n}\right) .
$$

The action of the permutation group $S_{n}$ on the Hilbert space $\mathcal{H}$ is specified by the unitary operator $U(\sigma) \in \mathcal{L}(\mathcal{H})$ that acts trivially on the Hilbert space $\mathcal{H}_{N}$, i.e.,

$$
U(\sigma)=\mathbb{1}_{N} \otimes U_{e}(\sigma),
$$

where $U_{e}(\sigma) \in \mathcal{L}\left(\mathcal{H}_{e}^{\otimes n}\right)$. The permutation group $S_{n}$ acts on a vector $\left|v_{1}, \ldots, v_{n}\right\rangle \equiv\left|v_{1}\right\rangle \otimes \cdots \otimes\left|v_{n}\right\rangle \in \mathcal{H}_{e}^{\otimes n}$ as

$$
U_{e}(\sigma)\left|v_{1}, \ldots, v_{n}\right\rangle=\left|v_{\sigma^{-1}(1)}, \ldots, v_{\sigma^{-1}(n)}\right\rangle .
$$

The relations (3) and (4) imply that

$$
U\left(\sigma_{1}\right) U\left(\sigma_{2}\right)=\omega\left(\sigma_{1}, \sigma_{2}\right) U\left(\sigma_{1} \sigma_{2}\right) \quad \forall \sigma_{1}, \sigma_{2} \in S_{n},
$$

where the phase factor is trivial, i.e., $\omega\left(\sigma_{1}, \sigma_{2}\right)=1$. The homomorphism $\sigma \mapsto U(\sigma)$ is a unitary representation of the permutation group $S_{n}$ acting on the Hilbert space $\mathcal{H}$.

In order to take into account the fermionic nature of the electrons, the Hilbert space describing the properties of the molecule, denoted $\mathcal{H}_{A+B}$ for reasons that shall become clear below, is defined as the isotypic component of type $\left[1^{n}\right]$ of the Hilbert space $\mathcal{H}$ with respect to the unitary representation $U(\sigma)$ of the permutation group $S_{n}$,

$$
\mathcal{H}_{A+B}=\mathcal{P}^{\left[1^{n}\right]} \mathcal{H}
$$

where the orthogonal projector $\mathcal{P}^{\left[1^{n}\right]} \in \mathcal{L}(\mathcal{H})$ is given by

$$
\mathcal{P}^{\left[1^{n}\right]}=\frac{1}{n !} \sum_{\sigma \in S_{n}} \varepsilon(\sigma) U(\sigma)
$$

and $\varepsilon(\sigma) \in\{-1,1\}$ denotes the signature of the permutation $\sigma \in S_{n}$ [29], which satisfies the condition

$$
\varepsilon\left(\sigma_{1}\right) \varepsilon\left(\sigma_{2}\right)=\varepsilon\left(\sigma_{1} \sigma_{2}\right) \quad \forall \sigma_{1}, \sigma_{2} \in S_{n} .
$$

The position $\boldsymbol{R}_{\mu}$ and momentum $\boldsymbol{P}_{\mu}$ of the nuclei, where $\mu=1, \ldots, N$, belong to the space of linear operators $\mathcal{L}\left(\mathcal{H}_{N}\right)$. The position $\boldsymbol{r}_{v}$, momentum $\boldsymbol{p}_{v}$, and spin $\boldsymbol{s}_{v}$ of the electrons, where $v=1, \ldots, n$, belong to the space of linear operators
$\mathcal{L}\left(\mathcal{H}_{e}^{\otimes n}\right)$. As shown in Ref. [30], the position $\boldsymbol{R}_{\mu}$ and momentum $\boldsymbol{P}_{\mu}$ of the nuclei, are related to the rotated position $\boldsymbol{R}_{\mu}(\boldsymbol{\omega})$ and rotated momentum $\boldsymbol{P}_{\mu}(\boldsymbol{\omega})$ of the nuclei by the rotation operator $R(\omega)$ that is a function of the molecular orientation operator $\omega$, i.e.,

$$
\begin{aligned}
& \boldsymbol{R}_{\mu}(\boldsymbol{\omega})=\mathrm{R}(\boldsymbol{\omega})^{-1} \cdot\left(\boldsymbol{R}_{\mu} \otimes \mathbb{1}_{e}^{\otimes n}-\mathcal{Q}\right), \\
& \boldsymbol{P}_{\mu}(\boldsymbol{\omega})=\frac{1}{2}\left\{\mathrm{R}(\boldsymbol{\omega}),\left(\boldsymbol{P}_{\mu} \otimes \mathbb{1}_{e}^{\otimes n}-\frac{M_{\mu}}{\mathcal{M}} \mathcal{P}\right)\right\},
\end{aligned}
$$

where $M_{\mu}$ is the mass of the nucleus $\mu$ and $\mathcal{M}$ is the mass of the molecule. The position $\mathcal{Q}$ and momentum $\mathcal{P}$ of the center of mass of the molecule belong to the space of linear operators $\mathcal{L}(\mathcal{H})$. The position $\boldsymbol{r}_{v}$, momentum $\boldsymbol{p}_{v}$, and spin $\boldsymbol{s}_{v}$ of the electrons are related the rotated position $\boldsymbol{r}_{\nu}(\boldsymbol{\omega})$, rotated momentum $\boldsymbol{p}_{v}(\boldsymbol{\omega})$, and rotated spin $\boldsymbol{s}_{v}(\boldsymbol{\omega})$ of the electrons by the rotation operator $R(\omega)$, i.e.,

$$
\begin{aligned}
& \boldsymbol{r}_{v}(\boldsymbol{\omega})=\mathrm{R}(\boldsymbol{\omega})^{-1} \cdot\left(\mathbb{1}_{N} \otimes \boldsymbol{r}_{v}-\mathcal{Q}\right), \\
& \boldsymbol{p}_{v}(\boldsymbol{\omega})=\mathrm{R}(\boldsymbol{\omega}) \cdot\left(\mathbb{1}_{N} \otimes \boldsymbol{p}_{v}-\frac{m}{\mathcal{M}} \mathcal{P}\right), \\
& \boldsymbol{s}_{v}(\boldsymbol{\omega})=\mathrm{R}(\boldsymbol{\omega}) \cdot \boldsymbol{s}_{v},
\end{aligned}
$$

where $m$ is the mass of an electron. The positions $\boldsymbol{R}_{\mu}(\boldsymbol{\omega})$ and $\boldsymbol{r}_{v}(\boldsymbol{\omega})$ and the momenta $\boldsymbol{P}_{\mu}(\boldsymbol{\omega})$ and $\boldsymbol{p}_{v}(\boldsymbol{\omega})$ can be expressed in terms of internal properties. These properties include the molecular orientation $\omega$, the molecular orbital angular momentum $\boldsymbol{L}(\boldsymbol{\omega})$, the amplitudes of the molecular deformations $Q_{\alpha}$, the momenta of the molecular deformation $P_{\alpha}$ in the modes $\alpha=1, \ldots, 3 N-6$, the electronic positions $\boldsymbol{q}_{v}$, and the electronic momenta $\boldsymbol{p}_{v}$, where $v=1, \ldots, n$.

We decompose the molecule into a system $A$ and a bath $B$, such that the system $A$ consists of the molecular deformation and orientation properties and the electronic spin properties and the bath $B$ consists of the electronic spatial properties. The Hilbert space $\mathcal{H}$ describing the properties of the molecule is expressed as the tensor product of Hilbert space describing different types of internal properties, i.e.,

$$
\mathcal{H}=\left(\mathcal{H}_{\text {def }} \otimes \mathcal{H}_{\text {ori }} \otimes \mathcal{H}_{s}^{\otimes n}\right) \otimes \mathcal{H}_{o}^{\otimes n}
$$

The Hilbert space $\mathcal{H}_{\text {def }}=L^{2}\left(\mathbb{R}^{3 N-6}, d x^{3 N-6}\right)$ describes the molecular deformations, the Hilbert space $\mathcal{H}_{\text {ori }}=L^{2}(\operatorname{SO}(3)$, $\sin \theta d \psi d \theta d \varphi)$ describes the molecular orientation, with $\psi \in$ $[0,2 \pi), \theta \in[0, \pi], \varphi \in[0,2 \pi)$, the Hilbert space $\mathcal{H}_{s}^{\otimes n}=\mathbb{C}^{2 \otimes n}$ describes the electronic spin properties, and the Hilbert space $\mathcal{H}_{o}^{\otimes n}=L^{2}\left(\mathbb{R}^{3 n}, d^{3 n} x\right)$ describes the electronic spatial properties.

The positions $\boldsymbol{R}_{\mu}(\boldsymbol{\omega})$ and $\boldsymbol{r}_{v}(\boldsymbol{\omega})$ and the momenta $\boldsymbol{P}_{\mu}(\boldsymbol{\omega})$ and $\boldsymbol{p}_{v}(\boldsymbol{\omega})$ belong to the fiber bundle $\mathcal{L}(\mathcal{H}) \times \mathcal{L}\left(\mathcal{L}\left(\mathcal{H}_{\text {ori }}\right)\right)$. The rotation operator $R(\omega)$, which is a function of the molecular orientation operator $\omega$ and thus plays the role of a superoperator, belongs to fiber $\mathcal{L}\left(\mathcal{L}\left(\mathcal{H}_{\text {ori }}\right)\right)$. The amplitudes and momenta of the molecular deformations $Q_{\alpha}$ and $P_{\alpha}$ belong to the space of linear operators $\mathcal{L}\left(\mathcal{H}_{\mathrm{def}}\right)$. The molecular orbital angular momentum $\boldsymbol{L}(\boldsymbol{\omega})$ belongs to the space of linear operators $\mathcal{L}\left(\mathcal{H}_{\text {ori }}\right)$. The spins $\boldsymbol{s}_{v}(\boldsymbol{\omega})$ belong to the space of linear operators $\mathcal{L}\left(\mathcal{H}_{s}^{\otimes n}\right)$. The electronic positions and momenta $\boldsymbol{q}_{v}$ and $\boldsymbol{p}_{v}$ belong to the space of linear operators $\mathcal{L}\left(\mathcal{H}_{o}^{\otimes n}\right)$. 
According to our choice of system $A$ and bath $B$, the Hilbert space (11) is recast as

$$
\mathcal{H}=\mathcal{H}_{A} \otimes \mathcal{H}_{B},
$$

where the Hilbert spaces $\mathcal{H}_{A}$ and $\mathcal{H}_{B}$ are expressed as

$$
\mathcal{H}_{A}=\mathcal{H}_{\text {def }} \otimes \mathcal{H}_{\text {ori }} \otimes \mathcal{H}_{s}^{\otimes n} \quad \text { and } \quad \mathcal{H}_{B}=\mathcal{H}_{o}^{\otimes n} .
$$

The Hilbert spaces $\mathcal{H}_{A}$ and $\mathcal{H}_{B}$ are expressed as the direct sum of the subspaces $\mathcal{H}_{A}^{\lambda}$ and $\mathcal{H}_{B}^{\lambda^{\prime}}$, which are invariant with respect to the unitary representations $\sigma \mapsto U(\sigma)$ of the permutation group $S_{n}$ for all $\sigma \in S_{n}$, respectively,

$$
\mathcal{H}_{A}=\bigoplus_{\lambda} \mathcal{H}_{A}^{\lambda} \quad \text { and } \quad \mathcal{H}_{B}=\bigoplus_{\lambda^{\prime}} \mathcal{H}_{B}^{\lambda^{\prime}} .
$$

The Hilbert spaces $\mathcal{H}_{A}^{\lambda}$ and $\mathcal{H}_{B}^{\lambda^{\prime}}$ are called the isotypic components of types $\lambda$ and $\lambda^{\prime}$ of the permutation group $S_{n}$ in the Hilbert spaces $\mathcal{H}_{A}$ and $\mathcal{H}_{B}$, respectively. The substitution of the relations (14) into the expressions (12) of the Hilbert space $\mathcal{H}$ yields

$$
\mathcal{H}=\bigoplus_{\lambda, \lambda^{\prime}} \mathcal{H}_{A}^{\lambda} \otimes \mathcal{H}_{B}^{\lambda^{\prime}}
$$

The substitution of the relations (15) into the expression (6) for the Hilbert space $\mathcal{H}_{A+B}$ of the molecule yields

$$
\mathcal{H}_{A+B}=\mathcal{P}^{\left[1^{n}\right]} \bigoplus_{\lambda, \lambda^{\prime}} \mathcal{H}_{A}^{\lambda} \otimes \mathcal{H}_{B}^{\lambda^{\prime}}
$$

We seek to identify the tensorial products $\mathcal{H}_{A}^{\lambda} \otimes \mathcal{H}_{B}^{\lambda^{\prime}}$ that do not vanish after projection onto the isotypic component of type $\left[1^{n}\right]$ of the permutation group $S_{n}$ in the Hilbert space $\mathcal{H}$. In order to do so, we begin by writing the tensor product of the irreducible representations $D^{(\lambda)}$ and $D^{\left(\lambda^{\prime}\right)}$ of the permutation group $S_{n}$ acting on the tensor product of the Hilbert spaces $\mathcal{H}_{A}^{\lambda}$ and $\mathcal{H}_{B}^{\lambda^{\prime}}$ as the direct sum of the irreducible representations $D^{\left(\lambda^{\prime \prime}\right)}$ of the permutation group $S_{n}$ acting on the Hilbert space $\mathcal{H}_{A+B}$, i.e.,

$$
D^{(\lambda)} \otimes D^{\left(\lambda^{\prime}\right)}=\bigoplus_{\lambda^{\prime \prime}} a_{\lambda^{\prime \prime}} D^{\left(\lambda^{\prime \prime}\right)},
$$

where $a_{\lambda^{\prime \prime}}$ is the multiplicity of the irreducible representation $D^{\left(\lambda^{\prime \prime}\right)}$. In particular, for the type $\lambda^{\prime \prime}=\left[1^{n}\right]$, the multiplicity $a_{\left[1^{n}\right]}$ of the irreducible representation $D^{\left[1^{n}\right]}$ satisfies the orthogonality condition $[32,33]$,

$$
\begin{aligned}
a_{\left[1^{n}\right]} & =\frac{1}{n !} \sum_{\sigma \in S_{n}} \chi_{\left[1^{n}\right]}(\sigma)^{*} \chi_{\lambda}(\sigma) \chi_{\lambda^{\prime}}(\sigma) \\
& =\frac{1}{n !} \sum_{\sigma \in S_{n}} \chi_{\tilde{\lambda}}(\sigma)^{*} \chi_{\lambda^{\prime}}(\sigma)=\delta_{\tilde{\lambda} \lambda^{\prime}},
\end{aligned}
$$

where $\chi_{\left[1^{n}\right]}, \chi_{\lambda}, \chi_{\lambda^{\prime}}$, and $\chi_{\tilde{\lambda}}$ are the characters of the irreducible representations $D^{\left[1^{n}\right]}, D^{(\lambda)}, D^{\left(\lambda^{\prime}\right)}$, and $D^{(\tilde{\lambda})}$, and $\tilde{\lambda}$ is the type of the irreducible representation $D^{(\tilde{\lambda})}$ of the permutation group $S_{n}$ that is dual to $D^{(\lambda)}$. Thus, the expression (16) of the Hilbert space $\mathcal{H}_{A+B}$ of the molecule reduces to

$$
\mathcal{H}_{A+B}=\mathcal{P}^{\left[1^{n}\right]} \bigoplus_{\lambda} \mathcal{H}_{A}^{\lambda} \otimes \mathcal{H}_{B}^{\tilde{\lambda}}
$$

The dual irreducible representation $D^{(\tilde{\lambda})}$ is entirely determined by the irreducible representation $D^{(\lambda)}$. Thus, in the expression (19) of the Hilbert space $\mathcal{H}_{A+B}$, the direct sum is restricted to the type $\lambda$ only [29]. In order to account explicitly for the multiplicities $n_{A}^{\lambda}$ and $n_{B}^{\tilde{\lambda}}$ of the irreducible representations $D^{(\lambda)}$ and $D^{(\tilde{\lambda})}$ in the Hilbert spaces $\mathcal{H}_{A}^{\lambda}$ and $\mathcal{H}_{B}^{\tilde{\lambda}}$, respectively, the latter can be expressed as the direct sum of the Hilbert spaces $\mathcal{H}_{A a}^{\lambda}$ and $\mathcal{H}_{B b}^{\tilde{\lambda}}$, i.e.,

$$
\mathcal{H}_{A}^{\lambda}=\bigoplus_{a=1}^{n_{A}^{\lambda}} \mathcal{H}_{A a}^{\lambda} \quad \text { and } \quad \mathcal{H}_{B}^{\tilde{\lambda}}=\bigoplus_{b=1}^{n_{B}^{\tilde{\lambda}}} \mathcal{H}_{B b}^{\tilde{\lambda}} .
$$

The substitution of the relations (20) into the expression (19) of the Hilbert space $\mathcal{H}_{A+B}$ of the molecular space yields

$$
\mathcal{H}_{A+B}=\mathcal{P}^{\left[1^{n}\right]} \bigoplus_{\lambda} \bigoplus_{a=1}^{n_{A}^{\lambda}} \bigoplus_{b=1}^{n_{B}^{\tilde{x}}} \mathcal{H}_{A a}^{\lambda} \otimes \mathcal{H}_{B b}^{\tilde{\lambda}} .
$$

\section{B. Irreducible representations of the permutation group and spin quantum numbers}

An irreducible representation $D^{(\lambda)}$ of the permutation group $S_{n}$ of type $\lambda$ is associated with a partition of the integer $n$. A Young diagram is a graphical representation of a partition of $n$, where the integers of the partition are listed by decreasing order [34]. It consists of $n$ cells arranged in columns and rows where the number of cells in a row cannot exceed the number of cells in the previous row. The dual irreducible representation $D^{(\tilde{\lambda})}$ of the permutation group $S_{n}$ of type $\tilde{\lambda}$ is associated with the transposed Young diagram obtained by exchanging rows and columns. For example, the irreducible representation $D^{[n]}$ consists of $n$ cells on the first row, and the irreducible representation $D^{\left[1^{n}\right]}$, where $\left[1^{n}\right]=[1,1, \ldots, 1]$ is a partition of $n$, consists of $n$ cells in the first column. The irreducible representation $D^{\left[1^{n}\right]}$ is the dual of the irreducible representation $D^{[n]}$.

Since the spin properties of a single electron are described by the two-dimensional Hilbert space $\mathcal{H}_{s}=\mathbb{C}^{2}$, a Young diagram associated with an irreducible representation $D^{(\lambda)}$ of the permutation group $S_{n}$ cannot have more than two rows [35]. The type $\lambda$ is therefore determined by a partition $\lambda=\left[\lambda_{1}, \lambda_{2}\right]$ of $n$, where $\lambda_{1}$ and $\lambda_{2}$ are integers with $\lambda_{1} \geqslant \lambda_{2}$. The Young diagrams associated with the irreducible representations $D^{(\lambda)}$ of the permutation group $S_{n}$ of type $\lambda=\left[\lambda_{1}, \lambda_{2}\right]$ are illustrated in Fig. 1.

The dimension $d_{\lambda}$ of the irreducible representation $D^{(\lambda)}$ of the permutation group $S_{n}$ of type $\lambda=\left[\lambda_{1}, \lambda_{2}\right]$ is given by [29]

$$
d_{\lambda}=\frac{\lambda_{1}-\lambda_{2}+1}{\lambda_{1}+1} \frac{n !}{\lambda_{1} ! \lambda_{2} !} .
$$

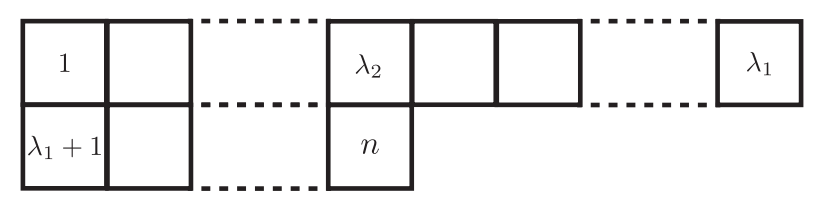

FIG. 1. Young diagram with $\lambda_{1}$ cells in the first row and $\lambda_{2}$ cells in the second row associated with the partition $\lambda=\left[\lambda_{1}, \lambda_{2}\right]$ of $n$. 
The multiplicity $n_{A}^{\lambda}$ of the irreducible representation $D^{(\lambda)}$ of the permutation group $S_{n}$ in the Hilbert space $\mathcal{H}_{A}^{\lambda}$ is given by

$$
n_{A}^{\lambda}=\lambda_{1}-\lambda_{2}+1 \text {. }
$$

The dimensions $d_{\lambda}$ and $d_{\tilde{\lambda}}$ of the irreducible representations of the permutation group $S_{n}$ of types $\lambda=\left[\lambda_{1}, \lambda_{2}\right]$ and $\tilde{\lambda}=\left[2^{\lambda_{2}}, 1^{\lambda_{1}-\lambda_{2}}\right]$ are the same, i.e., $d_{\lambda}=d_{\tilde{\lambda}}[32]$.

There is a one-to-one correspondence between the type $\left[\lambda_{1}, \lambda_{2}\right]$ of an irreducible representation $D^{(\lambda)}$ of the permutation group $S_{n}$ and the electronic spin quantum number $s$,

$$
\lambda_{1}=\frac{n}{2}+s \quad \text { and } \quad \lambda_{2}=\frac{n}{2}-s,
$$

because the irreducible representations of the rotation group $\mathrm{SO}(3)$ commute with the irreducible representations of the permutations group $S_{n}$ [29]. Thus, for a fixed number $n$ of electrons, the type $\lambda$ of the irreducible representation $D^{(\lambda)}$ and the type $\tilde{\lambda}$ of the dual irreducible representation $D^{(\lambda)}$ of the permutation group $S_{n}$ are entirely determined by the electronic spin quantum number $s$,

$$
\begin{aligned}
& \lambda \equiv \lambda(s)=\left[\frac{n}{2}+s, \frac{n}{2}-s\right], \\
& \tilde{\lambda} \equiv \tilde{\lambda}(s)=\left[2^{\frac{n}{2}+s}, 1^{2 s}\right] \text { and } \tilde{\lambda}(s) \equiv \tilde{s}(s) \equiv \tilde{s} .
\end{aligned}
$$

Henceforth, we shall denote the type of the isotypic components $\mathcal{H}_{A}^{\lambda}$ and $\mathcal{H}_{B}^{\tilde{\lambda}}$ of the permutation group $S_{n}$ in the Hilbert spaces $\mathcal{H}_{A}$ and $\mathcal{H}_{B}$, respectively, using the electronic spin quantum number $s$ and the type $\tilde{s}$, which is entirely determined by $s$, i.e.,

$$
\mathcal{H}_{A}^{\lambda} \rightarrow \mathcal{H}_{A}^{s} \quad \text { and } \quad \mathcal{H}_{B}^{\tilde{\lambda}} \rightarrow \mathcal{H}_{B}^{\tilde{s}} .
$$

Thus, the decomposition (19) of the Hilbert space $\mathcal{H}_{A+B}$ associated with the molecule is recast as

$$
\mathcal{H}_{A+B}=\mathcal{P}^{\left[1^{n}\right]} \bigoplus_{s} \mathcal{H}_{A}^{s} \otimes \mathcal{H}_{B}^{\tilde{s}}
$$

Moreover, using the replacements

$$
\mathcal{H}_{A a}^{\lambda} \rightarrow \mathcal{H}_{A a}^{s} \quad \text { and } \quad \mathcal{H}_{B b}^{\tilde{\lambda}} \rightarrow \mathcal{H}_{B b}^{\tilde{s}},
$$

the decomposition (21) of the Hilbert space $\mathcal{H}_{A+B}$ is recast as

$$
\mathcal{H}_{A+B}=\mathcal{P}^{\left[1^{n}\right]} \bigoplus_{s} \bigoplus_{a=1}^{n_{A}^{s}} \bigoplus_{b=1}^{n_{B}^{\tilde{s}}} \mathcal{H}_{A a}^{s} \otimes \mathcal{H}_{B b}^{\tilde{s}},
$$

with the multiplicities $n_{A}^{\lambda} \rightarrow n_{A}^{s}$ and $n_{B}^{\tilde{\lambda}} \rightarrow n_{B}^{\tilde{s}}$. The substitution of the relations (24) into the expression (22) yields the dimension $d_{s}$ of the irreducible representation $D^{(s)}$ of the permutation group $S_{n}$,

$$
d_{s}=\frac{2 s+1}{\frac{n}{2}+s+1} \frac{n !}{\left(\frac{n}{2}+s\right) !\left(\frac{n}{2}-s\right) !},
$$

where $d_{s}=d_{\tilde{s}}$. Similarly, the substitution of the relations (24) into the expression (23) yields the multiplicity $n_{A}^{\lambda} \rightarrow n_{A}^{s}$ of the irreducible representation $D^{(s)}$ of the permutation group $S_{n}$ in the Hilbert space $\mathcal{H}_{A}^{s}$ and is expressed as

$$
n_{A}^{s}=2 s+1 \text {. }
$$

\section{Spaces of linear operators}

The decomposition (12) implies that the space of linear operators $\mathcal{L}(\mathcal{H})$ acting on the Hilbert space $\mathcal{H}$ can be decomposed as

$$
\mathcal{L}(\mathcal{H})=\mathcal{L}\left(\mathcal{H}_{A}\right) \otimes \mathcal{L}\left(\mathcal{H}_{B}\right),
$$

where $\mathcal{L}\left(\mathcal{H}_{A}\right)$ and $\mathcal{L}\left(\mathcal{H}_{B}\right)$ are the spaces of linear operators acting, respectively, on the Hilbert spaces $\mathcal{H}_{A}$ and $\mathcal{H}_{B}$. A unitary representation $U(\sigma) \in \mathcal{L}(\mathcal{H})$ of the permutation group $S_{n}$ in the Hilbert space $\mathcal{H}$ associated with the molecule decomposes as

$$
U(\sigma)=U_{A}(\sigma) \otimes U_{B}(\sigma)
$$

with the unitary representations $U_{A}(\sigma) \in \mathcal{L}\left(\mathcal{H}_{A}\right)$ and $U_{B}(\sigma) \in$ $\mathcal{L}\left(\mathcal{H}_{B}\right)$. Any observable of the molecule can be described by a linear operator $\mathcal{O} \in \mathcal{L}(\mathcal{H})_{A+B}$, which commutes with the unitary representation of the permutation group $S_{n}$ in the Hilbert space $\mathcal{H}$,

$$
[\mathcal{O}, U(\sigma)]=0 \quad \forall \sigma \in S_{n},
$$

since the $n$ electrons are indiscernible. The commutation relation (34) implies that the space of linear operators $\mathcal{L}(\mathcal{H})_{A+B}$ is given by the isotypic component of type $[n]$ of the permutation group $S_{n}$ in the space of linear operators $\mathcal{L}(\mathcal{H})$,

$$
\mathcal{L}(\mathcal{H})_{A+B}=\mathscr{P}^{[n]} \mathcal{L}(\mathcal{H}),
$$

where the orthogonal projector $\mathscr{P}^{[n]} \in \mathcal{L}(\mathcal{L}(\mathcal{H}))$ is a superoperator given by

$$
\mathscr{P}^{[n]}=\frac{1}{n !} \sum_{\sigma \in S_{n}} \mathscr{U}(\sigma)
$$

and the unitary representation $\mathscr{U}(\sigma) \in \mathcal{L}(\mathcal{L}(\mathcal{H}))$ is a superoperator that acts on the space of linear operators $\mathcal{L}(\mathcal{H})$ according to

$$
\mathscr{U}(\sigma) \mathcal{O}=U(\sigma) \mathcal{O} U(\sigma)^{-1} .
$$

Any observable of the system $A$ can be described by a linear operator $\mathcal{O}_{A} \in \mathcal{L}\left(\mathcal{H}_{A}\right)$, which commutes with the unitary representation of the permutation group $S_{n}$ in the Hilbert space $\mathcal{H}_{A}$,

$$
\left[\mathcal{O}_{A}, U_{A}(\sigma)\right]=0 \quad \forall \sigma \in S_{n} .
$$

Similarly, any observable of the system $A$ can be described by a linear operator $\mathcal{O}_{B} \in \mathcal{L}\left(\mathcal{H}_{B}\right)$, which commutes with the unitary representation of the permutation group $S_{n}$ in the Hilbert space $\mathcal{H}_{B}$,

$$
\left[\mathcal{O}_{B}, U_{B}(\sigma)\right]=0 \quad \forall \sigma \in S_{n} .
$$

The dynamics of the molecule is characterized by an Hamiltonian $H \in \mathcal{L}(\mathcal{H})_{A+B}$ such that

$$
H=H_{A} \otimes \mathbb{1}_{B}+\mathbb{1}_{A} \otimes H_{B}+H_{\mathrm{int}},
$$

where the Hamiltonian $H_{A} \in \mathcal{L}\left(\mathcal{H}_{A}\right)$ describes the free evolution of the system $A$, the Hamiltonian $H_{B} \in \mathcal{L}\left(\mathcal{H}_{B}\right)$ describes the free evolution of the bath $B$ and the Hamiltonian $H_{\text {int }} \in$ $\mathcal{L}(\mathcal{H})_{A+B}$ represents the interaction between the system $A$ and the bath $B$. According to the conditions (34)-(39), the Hamiltonians $H, H_{A}, H_{B}$, and $H_{\text {int }}$ commute with the corresponding 
unitary representations of the permutation group, i.e.,

$$
\begin{aligned}
& {[H, U(\sigma)]=0 \quad \forall \sigma \in S_{n},} \\
& {\left[H_{A}, U_{A}(\sigma)\right]=0 \quad \forall \sigma \in S_{n},} \\
& {\left[H_{B}, U_{B}(\sigma)\right]=0 \quad \forall \sigma \in S_{n},} \\
& {\left[H_{\text {int }}, U(\sigma)\right]=0 \quad \forall \sigma \in S_{n} .}
\end{aligned}
$$

The spaces of linear operators $\mathcal{L}\left(\mathcal{H}_{A}\right)$ and $\mathcal{L}\left(\mathcal{H}_{B}\right)$ are expressed as the direct sum of the of the subspaces $\mathcal{L}\left(\mathcal{H}_{A}\right)^{\mu}$ and $\mathcal{L}\left(\mathcal{H}_{B}\right)^{\mu^{\prime}}$, which are invariant with respect to the unitary representations $\sigma \mapsto \mathscr{U}(\sigma)$ of the permutation group $S_{n}$ for all $\sigma \in S_{n}$, respectively,

$$
\begin{aligned}
\mathcal{L}\left(\mathcal{H}_{A}\right) & =\bigoplus_{\mu} \mathcal{L}\left(\mathcal{H}_{A}\right)^{\mu}, \\
\mathcal{L}\left(\mathcal{H}_{B}\right) & =\bigoplus_{\mu^{\prime}} \mathcal{L}\left(\mathcal{H}_{B}\right)^{\mu^{\prime}} .
\end{aligned}
$$

The spaces of linear operators $\mathcal{L}\left(\mathcal{H}_{A}\right)^{\mu}$ and $\mathcal{L}\left(\mathcal{H}_{B}\right)^{\mu^{\prime}}$ are the isotypic components of types $\mu$ and $\mu^{\prime}$ of the permutation group $S_{n}$ in the spaces of linear operators $\mathcal{L}\left(\mathcal{H}_{A}\right)$ and $\mathcal{L}\left(\mathcal{H}_{B}\right)$, respectively. The substitution of the relations (42) into the decomposition (32) yields

$$
\mathcal{L}(\mathcal{H})=\bigoplus_{\mu, \mu^{\prime}} \mathcal{L}\left(\mathcal{H}_{A}\right)^{\mu} \otimes \mathcal{L}\left(\mathcal{H}_{B}\right)^{\mu^{\prime}}
$$

The substitution of the relation (43) in the expression (35) of the space of linear operators $\mathcal{L}(\mathcal{H})_{A+B}$ yields

$$
\mathcal{L}(\mathcal{H})_{A+B}=\mathscr{P}^{[n]} \bigoplus_{\mu, \mu^{\prime}} \mathcal{L}\left(\mathcal{H}_{A}\right)^{\mu} \otimes \mathcal{L}\left(\mathcal{H}_{B}\right)^{\mu^{\prime}}
$$

We seek to identify the tensorial products $\mathcal{L}\left(\mathcal{H}_{A}\right)^{\mu} \otimes \mathcal{L}\left(\mathcal{H}_{B}\right)^{\mu^{\prime}}$ that do not vanish after projection onto the isotypic component of type $[n]$ of the permutation group $S_{n}$ in the space of linear operators $\mathcal{L}(\mathcal{H})$. In order to do so, we begin by writing the tensor product of the irreducible representations $D^{(\mu)}$ and $D^{\left(\mu^{\prime}\right)}$ of the permutation group $S_{n}$ acting on the tensor product of the space of linear operators $\mathcal{L}\left(\mathcal{H}_{A}\right)^{\mu}$ and $\mathcal{L}\left(\mathcal{H}_{B}\right)^{\mu^{\prime}}$ as the direct sum of the irreducible representations $D^{\left(\mu^{\prime \prime}\right)}$ of the permutation group $S_{n}$ acting on the space of linear operators $\mathcal{L}(\mathcal{H})_{A+B}$, i.e.,

$$
D^{(\mu)} \otimes D^{\left(\mu^{\prime}\right)}=\bigoplus_{\mu^{\prime \prime}} a_{\mu^{\prime \prime}} D^{\left(\mu^{\prime \prime}\right)}
$$

where $a_{\mu^{\prime \prime}}$ is the multiplicity of the irreducible representation $D^{\left(\mu^{\prime \prime}\right)}$. In particular, for the type $\mu^{\prime \prime}=[n]$, the multiplicity $a_{[n]}$ of the irreducible representation $D^{[n]}$ satisfies the orthogonality condition $[32,33]$,

$$
\begin{aligned}
a_{[n]} & =\frac{1}{n !} \sum_{\sigma \in S_{n}} \chi_{[n]}(\sigma)^{*} \chi_{\mu}(\sigma) \chi_{\mu^{\prime}}(\sigma) \\
& =\frac{1}{n !} \sum_{\sigma \in S_{n}} \chi_{\mu}(\sigma)^{*} \chi_{\mu^{\prime}}(\sigma)=\delta_{\mu \mu^{\prime}},
\end{aligned}
$$

where $\chi_{[n]}, \chi_{\mu}$, and $\chi_{\mu^{\prime}}$ are the characters of the irreducible representations $D^{[n]}, D^{(\lambda)}$, and $D^{(\mu)}$, respectively. Thus, the expression (44) of the space of linear operators $\mathcal{L}(\mathcal{H})_{A+B}$ of the molecule reduces to

$$
\mathcal{L}(\mathcal{H})_{A+B}=\mathscr{P}^{[n]} \bigoplus_{\mu} \mathcal{L}\left(\mathcal{H}_{A}\right)^{\mu} \otimes \mathcal{L}\left(\mathcal{H}_{B}\right)^{\mu}
$$

In order to account explicitly for the multiplicities $N_{A}^{\mu}$ and $N_{B}^{\mu}$ of the irreducible representation $D^{(\mu)}$ in the spaces of linear operators $\mathcal{L}\left(\mathcal{H}_{A}\right)^{\mu}$ and $\mathcal{L}\left(\mathcal{H}_{B}\right)^{\mu}$, respectively, the latter can be expressed as the direct sum of the spaces of linear operators $\mathcal{L}\left(\mathcal{H}_{A}\right)_{a}^{\mu}$ and $\mathcal{L}\left(\mathcal{H}_{B}\right)_{b}^{\mu}$, i.e.,

$$
\begin{aligned}
\mathcal{L}\left(\mathcal{H}_{A}\right)^{\mu} & =\bigoplus_{a=1}^{N_{A}^{\mu}} \mathcal{L}\left(\mathcal{H}_{A}\right)_{a}^{\mu}, \\
\mathcal{L}\left(\mathcal{H}_{B}\right)^{\mu} & =\bigoplus_{b=1}^{N_{B}^{\mu}} \mathcal{L}\left(\mathcal{H}_{B}\right)_{b}^{\mu} .
\end{aligned}
$$

The substitution of the relations (20) into the expression (19) of the Hilbert space $\mathcal{H}_{A+B}$ of the molecule yields

$$
\mathcal{L}(\mathcal{H})_{A+B}=\mathscr{P}{ }^{[n]} \bigoplus_{\mu} \bigoplus_{a=1}^{N_{A}^{\mu}} \bigoplus_{b=1}^{N_{B}^{\mu}} \mathcal{L}\left(\mathcal{H}_{A}\right)_{a}^{\mu} \otimes \mathcal{L}\left(\mathcal{H}_{B}\right)_{b}^{\mu}
$$

\section{Orthonormal vector basis}

The state of the molecule is described by a ray of the appropriate subspace of the Hilbert space $\mathcal{H}_{A+B}$ associated with the molecule.

The Hilbert space $\mathcal{H}_{A}$ is supplied with an orthonormal vector basis $\{|a, s, i\rangle\}$, where $a$ is a list of numbers labeling the physical properties described by the Hilbert space $\mathcal{H}_{A}^{s}$ and $i=1, \ldots, d_{s}$. Similarly, the Hilbert space $\mathcal{H}_{B}$ is supplied with an orthonormal vector basis $\{|b, \tilde{s}, j\rangle\}$, where $b$ is a list of numbers labeling the physical properties described by the Hilbert space $\mathcal{H}_{B}^{\tilde{s}}$ and $i=1, \ldots, d_{s}$. The vectors $|a, s, i\rangle \in \mathcal{H}_{A}$ and $|b, \tilde{s}, j\rangle \in \mathcal{H}_{B}$ satisfy the orthonormality conditions, i.e.,

$$
\begin{aligned}
\left\langle a^{\prime}, s^{\prime}, i^{\prime} \mid a, s, i\right\rangle & =\delta^{a^{\prime} a} \delta^{s^{\prime} s} \delta_{i^{\prime} i}, \\
\left\langle b^{\prime}, \tilde{s}^{\prime}, j^{\prime} \mid b, \tilde{s}, j\right\rangle & =\delta^{b^{\prime} b} \delta^{\tilde{s}^{\prime} \tilde{s}} \delta_{j^{\prime} j} .
\end{aligned}
$$

In addition, the basis vectors are chosen such that the action of the permutation group $S_{n}$ on the sets of vectors $\{|a, s, i\rangle\}$ and $\{|b, \tilde{s}, j\rangle\}$ is given by

$$
\begin{aligned}
& U_{A}(\sigma)|a, s, k\rangle=\sum_{i=1}^{d_{s}}|a, s, i\rangle d_{i k}^{s}(\sigma), \\
& U_{B}(\sigma)|b, \tilde{s}, \ell\rangle=\sum_{j=1}^{d_{s}}|b, \tilde{s}, j\rangle d_{j \ell}^{\tilde{s}}(\sigma),
\end{aligned}
$$

where the coefficients $d_{i k}^{s}(\sigma)$ and $d_{j \ell}^{\tilde{s}}(\sigma)$ are the matrix elements of the so-called standard irreducible representations $d^{(s)}$ and $d^{(\tilde{s})}$ of the permutation group $S_{n}$. These coefficients satisfy the orthogonality relations

$$
\sum_{\sigma \in S_{n}} d_{i j}^{s^{\prime}}(\sigma)^{*} d_{k \ell}^{s}(\sigma)=\frac{n !}{d_{s}} \delta^{s^{\prime} s} \delta_{i k} \delta_{j \ell}
$$

and are always real. Henceforth, we consider that $d_{i j}^{s}(\sigma)=$ $d_{j i}^{s}(\sigma)^{*} \in \mathbb{R}$. 
The vectors $|a, s, k\rangle$ and $|b, \tilde{s}, \ell\rangle$ are chosen to be eigenvectors of the free Hamiltonians $H_{A}$ and $H_{B}$, respectively. The relevant spectra of the Hamiltonians $H_{A}$ and $H_{B}$ are assumed to be discrete. The eigenvalue equations are given by

$$
\begin{aligned}
& H_{A}|a, s, k\rangle=E_{a}^{s}|a, s, k\rangle, \\
& H_{B}|b, \tilde{s}, \ell\rangle=E_{b}^{\tilde{s}}|b, \tilde{s}, \ell\rangle,
\end{aligned}
$$

where $E_{a}^{s}$ is the energy of the system $A$ in the state $|a, s, k\rangle$ and $E_{b}^{\tilde{s}}$ is the energy of the bath in the state $|b, \tilde{s}, \ell\rangle$. The degeneracies of the energies $E_{a}^{s}$ and $E_{b}^{\tilde{s}}$ are at least equal to the dimension $d_{s}$.

The orthonormal basis of the Hilbert space $\mathcal{H}_{A+B}$ is deduced from the decomposition (19). The dimension of the irreducible representation $D^{\left[1^{n}\right]}$ of the permutation group $S_{n}$ is $d_{\left[1^{n]}\right.}=1$ since $d_{[n]}=1$ according to relation (22). According to the decomposition (29), a vector $|a, b, s\rangle \in \mathcal{H}_{A+B}$ is expressed as a linear combination of the tensor products of the vectors $|a, s, j\rangle \in \mathcal{H}_{A}^{s}$ and $|b, \tilde{s}, k\rangle \in \mathcal{H}_{B}^{\tilde{s}}$ as [29]

$$
|a, b, s\rangle=\sum_{j, k=1}^{d_{s}} c_{j k}^{s \tilde{s}\left[1^{n}\right]}|a, s, j\rangle \otimes|b, \tilde{s}, k\rangle,
$$

where $c_{j k 1}^{s \tilde{s}\left[^{n}\right]}$ are the Clebsch-Gordan coefficients associated with the projection of the Hilbert space $\mathcal{H}_{A}^{s} \otimes \mathcal{H}_{B}^{\tilde{s}}$ onto the Hilbert space $\mathcal{H}_{A+B}$. The set of vectors $\{|a, b, s\rangle\}$ is an orthonormal basis of the Hilbert space $\mathcal{H}_{A+B}$ associated with the molecule, which implies that the vectors satisfy the orthonormality condition

$$
\left\langle a^{\prime}, b^{\prime}, s^{\prime} \mid a, b, s\right\rangle=\delta^{a^{\prime} a} \delta^{b^{\prime} b} \delta^{s^{\prime} s} .
$$

The decomposition (54) and the orthonormality conditions (50) and (55) imply that the Clebsch-Gordan coefficients satisfy the condition

$$
\sum_{j, k=1}^{d_{s}} c_{j k}^{s \tilde{s}\left[1^{n}\right]^{*} *^{*}} c_{j k 1}^{s \tilde{s}\left[1^{n}\right]}=1,
$$

which is due to the fact that the Clebsch-Gordan coefficients are matrix elements of a unitary (or even orthogonal) transformation.

We introduce the partially isometric operators $\mathcal{P}_{A i j}^{s} \in \mathcal{L}\left(\mathcal{H}_{A}\right)$ and $\mathcal{P}_{B i j}^{\tilde{s}} \in \mathcal{L}\left(\mathcal{H}_{B}\right)$ mapping the vectors $|a, s, j\rangle$ and $|b, \tilde{s}, j\rangle$ onto the vectors $|a, s, i\rangle$ and $|b, \tilde{s}, i\rangle$, respectively, according to

$$
\begin{aligned}
& \mathcal{P}_{A i j}^{s^{\prime}}|a, s, j\rangle=\delta^{s^{\prime} s}|a, s, i\rangle, \\
& \mathcal{P}_{B i j}^{\tilde{s}^{\prime}}|b, \tilde{s}, j\rangle=\delta^{\tilde{s}^{\prime} \tilde{s}}|b, \tilde{s}, i\rangle .
\end{aligned}
$$

According to group theoretical developments [32], taking into account the relations (51) and (57) and the orthogonality relations (52), the operators $\mathcal{P}_{A i j}^{s}$ and $\mathcal{P}_{B i j}^{\tilde{s}}$ are expressed as

$$
\begin{aligned}
& \mathcal{P}_{A i j}^{s}=\frac{d_{s}}{n !} \sum_{\sigma \in S_{n}} d_{i j}^{s}(\sigma)^{*} U_{A}(\sigma), \\
& \mathcal{P}_{B i j}^{\tilde{s}}=\frac{d_{s}}{n !} \sum_{\sigma \in S_{n}} d_{i j}^{\tilde{s}}(\sigma)^{*} U_{B}(\sigma) .
\end{aligned}
$$

The properties of the operators $\mathcal{P}_{A i j}^{s}$ and $\mathcal{P}_{B i j}^{\tilde{s}}$ are

$$
\begin{array}{lll}
\mathcal{P}_{A i j}^{s \dagger}=\mathcal{P}_{A j i}^{s} & \text { and } \quad \mathcal{P}_{A i j}^{s^{\prime}} \mathcal{P}_{A k \ell}^{s}=\delta^{s^{\prime} s} \delta_{j k} \mathcal{P}_{A i \ell}^{s}, \\
\mathcal{P}_{B i j}^{\tilde{s} \dagger}=\mathcal{P}_{B j i}^{\tilde{s}} & \text { and } \quad \mathcal{P}_{B i j}^{\tilde{s}^{\prime}} \mathcal{P}_{B k \ell}^{\tilde{s}}=\delta^{\tilde{s}^{\prime} \tilde{s}} \delta_{j k} \mathcal{P}_{B i \ell}^{\tilde{s}} .
\end{array}
$$

The orthogonal projectors $\mathcal{P}_{A}^{s} \in \mathcal{L}\left(\mathcal{H}_{A}\right)$ and $\mathcal{P}_{B}^{\tilde{s}} \in \mathcal{L}\left(\mathcal{H}_{B}\right)$ are the traces of the operators $\mathcal{P}_{A i j}^{s}$ and $\mathcal{P}_{B i j}^{\tilde{s}}$,

$$
\begin{aligned}
& \mathcal{P}_{A}^{s}=\sum_{i=1}^{d_{s}} \mathcal{P}_{A i i}^{s}=\frac{d_{s}}{n !} \sum_{\sigma \in S_{n}} \chi_{s}(\sigma)^{*} U_{A}(\sigma), \\
& \mathcal{P}_{B}^{\tilde{s}}=\sum_{i=1}^{d_{s}} \mathcal{P}_{B i i}^{\tilde{s}}=\frac{d_{s}}{n !} \sum_{\sigma \in S_{n}} \chi_{\tilde{s}}(\sigma)^{*} U_{B}(\sigma),
\end{aligned}
$$

where the characters $\chi_{s}(\sigma)$ and $\chi_{\tilde{s}}(\sigma)$ are the trace of the irreducible representations $D^{(s)}$ and $D^{(\widetilde{s})}$ of the permutation group $S_{n}$,

$$
\chi_{s}(\sigma)=\sum_{i=1}^{d_{s}} d_{i i}^{s}(\sigma) \quad \text { and } \quad \chi_{\tilde{s}}(\sigma)=\sum_{i=1}^{d_{s}} d_{i i}^{\tilde{s}}(\sigma) .
$$

Taking into account the decomposition (33), the orthogonal projector $\mathcal{P}^{s}$ on the Hilbert space $\mathcal{H}_{A}^{s} \otimes \mathcal{H}_{B}^{\tilde{s}}$ is given by

$$
\mathcal{P}^{s}=\frac{d_{s}}{n !} \sum_{\sigma \in S_{n}} \chi_{s}(\sigma)^{*} U(\sigma) .
$$

Note that, according to the definitions (7) and (62), the projector $\mathcal{P}^{\left[1^{n}\right]}$ associated with the representation of type $\left[1^{n}\right]$ is characterized by the character $\chi_{\left[1^{n}\right]}(\sigma)=\varepsilon(\sigma)$.

The definitions (58) and (60) imply that operators $\mathcal{O}_{A} \in$ $\mathcal{L}\left(\mathcal{H}_{A}\right)$ and $\mathcal{O}_{B} \in \mathcal{L}\left(\mathcal{H}_{B}\right)$ that satisfy the commutation relations (38) and (39) commute with the operators and $\mathcal{P}_{A i j}^{s}$ and $\mathcal{P}_{A}^{s}$ and with the operators $\mathcal{P}_{B i j}^{\tilde{s}}$ and $\mathcal{P}_{B}^{\tilde{s}}$, respectively,

$$
\begin{array}{lll}
{\left[\mathcal{O}_{A}, \mathcal{P}_{A i j}^{s}\right]=0} & \text { and } & {\left[\mathcal{O}_{A}, \mathcal{P}_{A}^{s}\right]=0,} \\
{\left[\mathcal{O}_{B}, \mathcal{P}_{B i j}^{\tilde{s}}\right]=0} & \text { and } & {\left[\mathcal{O}_{B}, \mathcal{P}_{B}^{\tilde{s}}\right]=0 .}
\end{array}
$$

Using the properties (57) and (59) and the commutation relations (63), we obtain the matrix elements associated with the operators $\mathcal{O}_{A}$ and $\mathcal{O}_{B}$,

$$
\begin{aligned}
\left\langle a^{\prime}, s^{\prime}, j^{\prime}\left|\mathcal{O}_{A}\right| a, s, j\right\rangle & =\left\langle a^{\prime}, s^{\prime}, 1\left|\mathcal{P}_{A 1 j^{\prime}}^{s^{\prime}} \mathcal{O}_{A} \mathcal{P}_{A j 1}^{s}\right| a, s, 1\right\rangle \\
& =\delta^{s^{\prime} s} \delta_{j^{\prime} j}\left\langle a^{\prime}, s, 1\left|\mathcal{O}_{A}\right| a, s, 1\right\rangle, \\
\left\langle b^{\prime}, \tilde{s}^{\prime}, k^{\prime}\left|\mathcal{O}_{B}\right| b, \tilde{s}, k\right\rangle & =\left\langle b^{\prime}, \tilde{s}^{\prime}, 1\left|\mathcal{P}_{B 1 k^{\prime}}^{\tilde{s}^{\prime}} \mathcal{O}_{B} \mathcal{P}_{B k 1}^{\tilde{s}}\right| b, \tilde{s}, 1\right\rangle \\
& =\delta^{\tilde{s}^{\prime} \tilde{s}} \delta_{k^{\prime} k}\left\langle b^{\prime}, \tilde{s}, 1\left|\mathcal{O}_{B}\right| b, \tilde{s}, 1\right\rangle .
\end{aligned}
$$

Adopting the notation

$$
|a, s\rangle \equiv|a, s, 1\rangle \quad \text { and } \quad|b, \tilde{s}\rangle \equiv|b, \tilde{s}, 1\rangle,
$$

the reduced matrix elements (64) are recast explicitly as

$$
\begin{aligned}
& \left\langle a^{\prime}, s^{\prime}, j^{\prime}\left|\mathcal{O}_{A}\right| a, s, j\right\rangle=\delta^{s^{\prime} s} \delta_{j^{\prime} j}\left\langle a^{\prime}, s\left|\mathcal{O}_{A}\right| a, s\right\rangle, \\
& \left\langle b^{\prime}, \tilde{s}^{\prime}, k^{\prime}\left|\mathcal{O}_{B}\right| b, \tilde{s}, k\right\rangle=\delta^{\tilde{s}^{\prime} \tilde{s}} \delta_{k^{\prime} k}\left\langle b^{\prime}, \tilde{s}\left|\mathcal{O}_{B}\right| b, \tilde{s}\right\rangle .
\end{aligned}
$$


Taking into account the definition (60) and using the notation (65), the relations (57) require that

$$
\begin{aligned}
& \mathcal{P}_{A}^{s^{\prime}}|a, s\rangle=\delta^{s^{\prime} s}|a, s\rangle, \\
& \mathcal{P}_{B}^{\tilde{s}^{\prime}}|b, \tilde{s}\rangle=\delta^{\tilde{s}^{\prime} \tilde{s}}|b, \tilde{s}\rangle .
\end{aligned}
$$

\section{E. Linear operator basis}

The space of linear operators $\mathcal{L}\left(\mathcal{H}_{A}\right)$ is supplied with an orthonormal operator basis $\left\{\mathcal{O}_{A a j}^{\mu}\right\}$, where $a=1, \ldots, N_{a}^{\mu}$ and $j=1, \ldots, d_{\mu}$. Similarly, the space of linear operators $\mathcal{L}\left(\mathcal{H}_{B}\right)$ is supplied with an orthonormal operator basis $\left\{\mathcal{O}_{B b k}^{\mu}\right\}$, where $b=1, \ldots, N_{b}^{\mu}$ and $k=1, \ldots, d_{\mu}$. According to relation (22), the dimension of the irreducible representation $D^{[n]}$ of the permutation group $S_{n}$ is $d_{[n]}=1$. According to the decomposition (49), an operator $\mathcal{O} \in \mathcal{L}(\mathcal{H})_{A+B}$ can be expressed as a linear combination of the tensor products of the linear operators $\mathcal{O}_{A a j}^{\mu} \in \mathcal{L}\left(\mathcal{H}_{A}\right)_{a}^{\mu}$ and $\mathcal{O}_{B b k}^{\mu} \in \mathcal{L}\left(\mathcal{H}_{B}\right)_{b}^{\mu}$ as [29]

$$
\mathcal{O}=\bigoplus \bigoplus_{a=1}^{N_{a}^{\mu}} \bigoplus_{b=1}^{N_{b}^{\mu}} \sum_{j, k=1}^{d_{\mu}} \sqrt{d_{\mu}} c_{j k 1}^{\mu \mu[n]} \mathcal{O}_{A a j}^{\mu} \otimes \mathcal{O}_{B b k}^{\mu},
$$

where the Clebsch-Gordan coefficients $c_{j k 1}^{\mu \mu[n]}$ associated with the projection of the space of linear operators $\mathcal{L}\left(\mathcal{H}_{A}\right)^{\mu} \otimes$ $\mathcal{L}\left(\mathcal{H}_{B}\right)^{\mu}$ onto the space of linear operators $\mathcal{L}(\mathcal{H})_{A+B}$ are given by [32]

$$
c_{j k 1}^{\mu \mu[n]}=\frac{1}{\sqrt{d_{\mu}}} \delta_{j k} .
$$

This implies that the decomposition (68) reduces to

$$
\mathcal{O}=\bigoplus \bigoplus_{\mu} \bigoplus_{a=1}^{N_{a}^{\mu}} \sum_{b=1}^{N_{b}^{\mu}} \mathcal{O}_{A a j}^{\mu} \otimes \mathcal{O}_{B b j}^{\mu}
$$

The unitary representation $\mathscr{U}(\sigma)$ acting on the space of linear operators $\mathcal{L}(\mathcal{H})$ can be expressed as

$$
\mathscr{U}(\sigma)=\mathscr{U}_{A}(\sigma) \otimes \mathscr{U}_{B}(\sigma)
$$

where $\mathscr{U}_{A}(\sigma)$ and $\mathscr{U}_{B}(\sigma)$ are the unitary representations acting, respectively, on the spaces of linear operators $\mathcal{L}\left(\mathcal{H}_{A}\right)$ and $\mathcal{L}\left(\mathcal{H}_{B}\right)$. The action of the permutation group $S_{n}$ on the sets of linear operators $\left\{\mathcal{O}_{A j}^{\mu}\right\}$ and $\left\{\mathcal{O}_{B j}^{\mu}\right\}$ is expressed as

$$
\begin{aligned}
& \mathscr{U}_{A}(\sigma) \mathcal{O}_{A a j}^{\mu} \equiv U_{A}(\sigma) \mathcal{O}_{A a j}^{\mu} U_{A}(\sigma)^{-1}=\sum_{i=1}^{d_{\mu}} \mathcal{O}_{A a i}^{\mu} d_{i j}^{\mu}(\sigma), \\
& \mathscr{U}_{B}(\sigma) \mathcal{O}_{B b j}^{\mu} \equiv U_{B}(\sigma) \mathcal{O}_{B b j}^{\mu} U_{B}(\sigma)^{-1}=\sum_{i=1}^{d_{\mu}} \mathcal{O}_{B b i}^{\mu} d_{i j}^{\mu}(\sigma) .
\end{aligned}
$$

We introduce the partially isometric superoperators $\mathscr{P}_{A i j}^{\mu} \in$ $\mathcal{L}\left(\mathcal{L}\left(\mathcal{H}_{A}\right)\right)$ and $\mathscr{P}_{B i j}^{\mu} \in \mathcal{L}\left(\mathcal{L}\left(\mathcal{H}_{B}\right)\right)$ mapping the operators $\mathcal{O}_{A j}^{\mu}$ and $\mathcal{O}_{B j}^{\mu}$ onto the operators $\mathcal{O}_{A i}^{\mu}$ and $\mathcal{O}_{B i}^{\mu}$, respectively, according to

$$
\begin{aligned}
& \mathscr{P}_{A i j}^{\mu^{\prime}} \mathcal{O}_{A a j}^{\mu}=\delta^{\mu^{\prime} \mu} \mathcal{O}_{A a i}^{\mu}, \\
& \mathscr{P}_{B i j}^{\mu^{\prime}} \mathcal{O}_{B b j}^{\mu}=\delta^{\mu^{\prime} \mu} \mathcal{O}_{B b i}^{\mu} .
\end{aligned}
$$

According to group theoretical developments [32], taking into account the orthogonality relations (52) and the relations (72) and (73), the superoperators $\mathscr{P}_{A i j}^{\mu}$ and $\mathscr{P}_{B i j}^{\mu}$ are expressed as

$$
\begin{aligned}
& \mathscr{P}_{A i j}^{\mu}=\frac{d_{\mu}}{n !} \sum_{\sigma \in S_{n}} d_{i j}^{\mu}(\sigma)^{*} \mathscr{U}_{A}(\sigma), \\
& \mathscr{P}_{B i j}^{\mu}=\frac{d_{\mu}}{n !} \sum_{\sigma \in S_{n}} d_{i j}^{\mu}(\sigma)^{*} \mathscr{U}_{B}(\sigma) .
\end{aligned}
$$

The properties of the superoperators $\mathscr{P}_{A i j}^{\mu}$ and $\mathscr{P}_{B i j}^{\mu}$ are

$$
\begin{aligned}
& \mathscr{P}_{A i j}^{\mu \dagger}=\mathscr{P}_{A j i}^{\mu} \text { and } \quad \mathscr{P}_{A i j}^{\mu^{\prime}} \mathscr{P}_{A k \ell}^{\mu}=\delta^{\mu^{\prime} \mu} \delta_{j k} \mathscr{P}_{A i \ell}^{\mu}, \\
& \mathscr{P}_{B i j}^{\mu \dagger}=\mathscr{P}_{B j i}^{\mu} \text { and } \mathscr{P}_{B i j}^{\mu^{\prime}} \mathscr{P}_{B k \ell}^{\mu}=\delta^{\mu^{\prime} \mu} \delta_{j k} \mathscr{P}_{B i \ell}^{\mu} .
\end{aligned}
$$

The orthogonal projectors $\mathscr{P}_{A}^{\mu} \in \mathcal{L}\left(\mathcal{L}\left(\mathcal{H}_{A}\right)\right)$ and $\mathscr{P}_{B}^{\mu} \in$ $\mathcal{L}\left(\mathcal{L}\left(\mathcal{H}_{B}\right)\right)$ are the traces of the superoperators $\mathscr{P}_{A i j}^{\mu}$ and $\mathscr{P}_{B i j}^{\mu}$,

$$
\begin{aligned}
& \mathscr{P}_{A}^{\mu}=\sum_{i=1}^{d_{\mu}} \mathscr{P}_{A i i}^{\mu}=\frac{d_{\mu}}{n !} \sum_{\sigma \in S_{n}} \chi_{\mu}(\sigma)^{*} \mathscr{U}_{A}(\sigma), \\
& \mathscr{P}_{B}^{\mu}=\sum_{i=1}^{d_{\mu}} \mathscr{P}_{B i i}^{\mu}=\frac{d_{\mu}}{n !} \sum_{\sigma \in S_{n}} \chi_{\mu}(\sigma)^{*} \mathscr{U}_{B}(\sigma) .
\end{aligned}
$$

\section{GENERALIZED MASTER EQUATIONS}

In this section, we detail the general structure of the generalized master equations established in Ref. [29] for an $n$-electron system and express them in a suitable way to describe molecular dynamics.

\section{A. Quantum master equations}

The time-dependent density operator $\rho(t) \in \mathcal{L}(\mathcal{H})_{A+B}$ describes the statistical state of the molecule on the characteristic time scale $t$. It satisfies the von Neumann conditions [36], which require $\rho(t)$ to be a self-adjoint positive operator with unit trace, i.e.,

$$
\rho(t)=\rho(t)^{\dagger}, \quad \rho(t)^{2} \leqslant \rho(t), \quad \operatorname{Tr}[\rho(t)]=1 .
$$

The density operator of the molecule may be written as [29]

$$
\rho(t)=\rho_{A}(t) \otimes \rho_{B}(t)+\eta_{A B}(t),
$$

where the reduced density operators $\rho_{A}(t) \in \mathcal{L}\left(\mathcal{H}_{A}\right)$ and $\rho_{B}(t) \in \mathcal{L}\left(\mathcal{H}_{B}\right)$ describe the statistical state of the system $A$ and of the bath $B$, respectively, and the correlation operator $\eta_{A B}(t) \in \mathcal{L}(\mathcal{H})_{A+B}$ describes the statistical correlations between the system $A$ and the bath $B$. The reduced density operators $\rho_{A}(t)$ and $\rho_{B}(t)$ satisfy the von Neumann conditions [36],

$$
\begin{array}{lll}
\rho_{A}(t)=\rho_{A}(t)^{\dagger}, & \rho_{A}(t)^{2} \leqslant \rho_{A}(t), \quad \operatorname{Tr}_{A}\left[\rho_{A}(t)\right]=1, \\
\rho_{B}(t)=\rho_{B}(t)^{\dagger}, & \rho_{B}(t)^{2} \leqslant \rho_{B}(t), & \operatorname{Tr}_{B}\left[\rho_{B}(t)\right]=1 .
\end{array}
$$


The commutation relations (34)-(39) for the observables $\rho(t)$, $\rho_{A}(t), \rho_{B}(t)$, and $\eta_{A B}(t) \mathrm{read}$

$$
\begin{array}{ll}
{[\rho(t), U(\sigma)]=0} & \forall \sigma \in S_{n}, \\
{\left[\rho_{A}(t), U_{A}(\sigma)\right]=0} & \forall \sigma \in S_{n}, \\
{\left[\rho_{B}(t), U_{B}(\sigma)\right]=0} & \forall \sigma \in S_{n}, \\
{\left[\eta_{A B}(t), U(\sigma)\right]=0} & \forall \sigma \in S_{n} .
\end{array}
$$

We shall now express the reduced density operators $\rho_{A}(t)$ and $\rho_{B}(t)$ as direct sums of square block operators and the correlation operator $\eta_{A B}(t)$ as a direct sum of rectangular block operators. The reduced density operators $\rho_{A}(t) \in \mathcal{L}\left(\mathcal{H}_{A}\right)$ and $\rho_{B}(t) \in \mathcal{L}\left(\mathcal{H}_{B}\right)$ can be decomposed into square blocks operators according to

$$
\begin{aligned}
& \rho_{A}(t)=\left.\bigoplus_{s} \rho_{A}^{s}(t) \equiv \bigoplus_{s} \mathcal{P}_{A}^{s} \rho_{A}(t) \mathcal{P}_{A}^{s}\right|_{\mathcal{H}_{A}^{s} \rightarrow \mathcal{H}_{A}^{s}}, \\
& \rho_{B}(t)=\left.\bigoplus_{\tilde{s}} \rho_{B}^{\tilde{s}}(t) \equiv \bigoplus_{\tilde{s}} \mathcal{P}_{B}^{\tilde{s}} \rho_{B}(t) \mathcal{P}_{B}^{\tilde{s}}\right|_{\mathcal{H}_{B}^{\tilde{s}} \rightarrow \mathcal{H}_{B}^{\tilde{s}}} .
\end{aligned}
$$

Decomposition (81) is performed here in order to express later the master equations as a set of coupled equations for block operators defined by the spin quantum number $s$. Taking into account the relation (72), the correlation operator $\eta_{A B}(t) \in$ $\mathcal{L}(\mathcal{H})_{A+B}$ can be decomposed as

$$
\eta_{A B}(t)=\bigoplus_{\mu} \bigoplus_{a=1}^{N_{a}^{\mu}} \bigoplus_{b=1}^{N_{b}^{\mu}} \sum_{j=1}^{d_{\mu}} \eta_{A a j}^{\mu}(t) \otimes \eta_{B b j}^{\mu}(t) .
$$

According to the relation (72), the action of the permutation group $S_{n}$ on the reduced correlation operators $\eta_{A a j}^{\mu}(t)$ and $\eta_{B b j}^{\mu}(t)$ is expressed as

$$
\begin{aligned}
U_{A}(\sigma) \eta_{A a j}^{\mu}(t) U_{A}(\sigma)^{-1} & =\sum_{i=1}^{d_{\mu}} \eta_{A a i}^{\mu}(t) d_{i j}^{\mu}(\sigma), \\
U_{B}(\sigma) \eta_{B b j}^{\mu}(t) U_{B}(\sigma)^{-1} & =\sum_{i=1}^{d_{\mu}} \eta_{B b i}^{\mu}(t) d_{i j}^{\mu}(\sigma) .
\end{aligned}
$$

Similarly, the operators $\eta_{A a j}^{\mu}(t)$ and $\eta_{B b j}^{\mu}(t)$ can be decomposed into rectangular block operators according to

$$
\begin{aligned}
& \eta_{A a j}^{\mu}(t)=\left.\bigoplus_{s, s^{\prime}} \eta_{A a j}^{s \mu s^{\prime}}(t) \equiv \bigoplus_{s, s^{\prime}} \mathcal{P}_{A}^{s} \eta_{A a j}^{\mu}(t) \mathcal{P}_{A}^{s^{\prime}}\right|_{\mathcal{H}_{A}^{s^{\prime}} \rightarrow \mathcal{H}_{A}^{s}}, \\
& \eta_{B b j}^{\mu}(t)=\left.\bigoplus_{\tilde{s}, \tilde{s}^{\prime}} \eta_{B b j}^{\tilde{s} \mu \tilde{s}^{\prime}}(t) \equiv \bigoplus_{\tilde{s}, \tilde{s}^{\prime}} \mathcal{P}_{B}^{\tilde{s}} \eta_{B b j}^{\mu}(t) \mathcal{P}_{B}^{\tilde{s}^{\prime}}\right|_{\mathcal{H}_{B}^{\tilde{s}^{\prime}} \rightarrow \mathcal{H}_{B}^{\tilde{s}}}
\end{aligned}
$$

Taking into account the decomposition (84), the expression (82) can be recast as

$$
\eta_{A B}(t)=\bigoplus_{\mu} \bigoplus_{a=1}^{N_{a}^{\mu}} \bigoplus_{b=1}^{N_{b}^{\mu}} \bigoplus_{s, s^{\prime}} \sum_{j=1}^{d_{\mu}} \eta_{A j}^{s \mu s^{\prime}}(t) \otimes \eta_{B j}^{\tilde{s} \mu \tilde{s}^{\prime}}(t) .
$$

The substitution of the decompositions (81) and (85) into the expression (78) yields

$$
\begin{aligned}
\rho(t)= & \bigoplus_{s} \rho_{A}^{s}(t) \otimes \rho_{B}^{\tilde{s}}(t) \\
& \bigoplus_{\mu} \bigoplus_{a=1}^{N_{a}^{\mu}} \bigoplus_{b=1}^{N_{b}^{\mu}} \bigoplus_{s, s^{\prime}} \sum_{j=1}^{d_{\mu}} \eta_{A a j}^{s \mu s^{\prime}}(t) \otimes \eta_{B b j}^{\tilde{s} \mu \tilde{s}^{\prime}}(t) .
\end{aligned}
$$

The reduced density operators $\rho_{A}^{s}(t)$ and $\rho_{B}^{\tilde{s}}(t)$ are the partial traces of the density operator $\rho(t)$ restricted to the Hilbert spaces $\mathcal{H}_{A}^{s}$ and $\mathcal{H}_{B}^{\tilde{s}}$, respectively,

$$
\rho_{A}^{s}(t)=\operatorname{Tr}_{B}^{\tilde{s}}[\rho(t)] \quad \text { and } \quad \rho_{B}^{\tilde{s}}(t)=\operatorname{Tr}_{A}^{s}[\rho(t)] .
$$

In order for the reduced density operators (87) to be expressed as the partial trace of the density operator (86), the correlation operator has to satisfy the conditions,

$$
\operatorname{Tr}_{A}^{s}\left[\eta_{A B}(t)\right]=0 \quad \text { and } \quad \operatorname{Tr}_{B}^{\tilde{s}}\left[\eta_{A B}(t)\right]=0 .
$$

The von Neumann conditions (77) on the density operator $\rho(t)$, the definitions (87) of the reduced density operators $\rho_{A}^{s}(t)$ and $\rho_{B}^{\tilde{s}}(t)$ and the properties (88) of the correlation operator imply that the reduced density operators satisfy the von Neumann conditions; i.e.,

$$
\begin{array}{ll}
\rho_{A}^{s}(t)=\rho_{A}^{s}(t)^{\dagger}, & \rho_{A}^{s}(t)^{2} \leqslant \rho_{A}^{s}(t), \quad \sum_{s} \operatorname{Tr}_{A}^{s}\left[\rho_{A}^{s}(t)\right]=1, \\
\rho_{B}^{\tilde{s}}(t)=\rho_{B}^{\tilde{s}}(t)^{\dagger}, & \rho_{B}^{\tilde{s}}(t)^{2} \leqslant \rho_{B}^{\tilde{s}}(t), \quad \sum_{\tilde{s}} \operatorname{Tr}_{B}^{\tilde{s}}\left[\rho_{B}^{\tilde{s}}(t)\right]=1 .
\end{array}
$$

The master equation for the evolution of the reduced density operator $\rho_{A}^{s}(t)$ of the system $A$ interacting with the bath $B$ and with an external field reads [29]

$$
\begin{aligned}
\frac{d}{d t} \rho_{A}^{s}(t)= & \frac{i}{\hbar}\left[\rho_{A}^{s}(t), H_{A}^{s}+\Delta H_{A}^{s}+H_{\mathrm{ext}}^{s}(t)\right] \\
& -\frac{1}{\hbar}\left\{\rho_{A}^{s}(t), G_{A}^{s}\right\}+\sum_{s^{\prime}} \Gamma_{s}^{s^{\prime}}\left(\rho_{A}^{s^{\prime}}(t)\right),
\end{aligned}
$$

where $\Delta H_{A}^{s}$ is a self-adjoint operator, $H_{\mathrm{ext}}^{s}(t)$ is the external Hamiltonian, $G_{A}^{s} \geqslant 0$ is a self-adjoint and positive definite operator, and $\Gamma_{s}^{s^{\prime}}\left[\rho_{A}^{s^{\prime}}(t)\right]$ is a linear operator. The external Hamiltonian $H_{\mathrm{ext}}^{s}(t)$ is sufficiently slowly varying on the characteristic time scale $t$. The Hamiltonian $H_{A}^{s}$ is related to the Hamiltonian $H_{A}$ by

$$
H_{A}^{s}=\left.\mathcal{P}_{A}^{s} H_{A} \mathcal{P}_{A}^{s}\right|_{\mathcal{H}_{A}^{s} \rightarrow \mathcal{H}_{A}^{s}} .
$$

The operators $\Delta H_{A}^{s}$ and $G_{A}^{s}$ commute with the Hamiltonian $H_{A}^{s}$, i.e.,

$$
\left[\Delta H_{A}^{s}, H_{A}^{s}\right]=0 \quad \text { and } \quad\left[G_{A}^{s}, H_{A}^{s}\right]=0 .
$$

The operators $\Delta H_{A}^{s}, G_{A}^{s}$, and the superoperator $\Gamma_{s}^{s^{\prime}}\left[\rho_{A}^{s^{\prime}}(t)\right]$ account for the influence of the system "A" on the bath $B$. The "self-energy" operator $\Delta H_{A}^{s}$ describes the shift of the energy levels of the system $A$, the operator $G_{A}^{s}$ ensures the conservation of the unit trace of the operator $\rho_{A}(t)$, and the "fluctuation and dissipation" superoperator $\Gamma_{s}^{s^{\prime}}\left[\rho_{A}^{s^{\prime}}(t)\right]$ accounts for fluctuations and dissipation. The operators $\rho_{A}^{s}(t)$, $H_{A}^{s}, \Delta H_{A}^{s}, H_{\text {ext }}^{s}(t)$, and $G_{A}^{s}$ belong to the space $\mathcal{L}\left(\mathcal{H}_{A}\right)$. The 
superoperator $\Gamma_{s}^{s^{\prime}}\left[\rho_{A}^{s^{\prime}}(t)\right]$ belongs to the space $\mathcal{L}\left(\mathcal{L}\left(\mathcal{H}_{A}\right)\right)$. This superoperator couples the evolution equations for the reduced density operators $\rho_{A}^{s}(t)$ and $\rho_{A}^{s^{\prime}}(t)$.

The master equation (90) was developed by the authors in order to describe the dynamics of a system $A$ interacting with a bath $B$ on a time interval $\Delta t_{A}$ that is sufficiently large compared to the correlation time $\tau_{B}^{\text {corr }}$, defined as the threshold above which the correlation functions of the bath are negligible, and sufficiently small compared to the evolution time $\tau_{B}^{\text {evo }}$, defined as the threshold below which the time evolution of the bath is negligible,

$$
\tau_{B}^{\text {corr }} \ll \Delta t_{A} \ll \tau_{B}^{\text {evo }} .
$$

The physical validity of the master equation (92) is guaranteed provided the statistical correlations between the system $A$ and the bath $B$ are sufficiently small. Thus, if the molecule is isolated, the master equation will be valid for a sufficiently small time interval $\Delta t_{A}$. However, if electromagnetic fields are included in the system, then the energy dissipation through radiative processes allows the bath to be kept close to equilibrium, thus ensuring that the condition (93) is satisfied.

\section{B. Time evolution of the populations and coherences}

According to our choice of orthonormal basis (53) and taking into account the notation (65), the vectors $|a, s\rangle$ and $|b, \tilde{s}\rangle$ are eigenvectors of the Hamiltonians $H_{A}$ and $H_{B}$, respectively, i.e.,

$$
\begin{aligned}
& H_{A}|a, s\rangle=E_{a}^{s}|a, s\rangle, \\
& H_{B}|b, \tilde{s}\rangle=E_{b}^{\tilde{s}}|b, \tilde{s}\rangle .
\end{aligned}
$$

The bath $B$ is close to equilibrium on the relevant time interval, which implies that the time-dependent reduced density operator $\rho_{B}^{\tilde{s}}(t)$ is the sum of a time-independent density operator $\rho_{B}^{\tilde{s}}$ that describes the equilibrium state and a time-dependent density operator $\delta \rho_{B}^{\tilde{s}}(t)$ that describes the deviations around the equilibrium state, i.e.,

$$
\rho_{B}^{\tilde{s}}(t)=\rho_{B}^{\tilde{s}}+\delta \rho_{B}^{\tilde{s}}(t) .
$$

The eigenvalue equation for the density operator $\rho_{B}^{\tilde{s}}$ is given by

$$
\rho_{B}^{\tilde{s}}|b, \tilde{s}\rangle=p_{b}^{\tilde{s}}|b, \tilde{s}\rangle,
$$

where $p_{b}^{\tilde{s}}$ represents the "population" of an energy level $E_{b}^{\tilde{s}}$. The commutation relation (92) and the eigenvalue equation (94) imply that the matrix elements associated with the operator $G_{A}^{s}$ are defined as

$$
G_{a^{\prime} a}^{s} \equiv \delta_{a^{\prime} a}\left\langle a, s\left|G_{A}^{s}\right| a, s\right\rangle .
$$

The matrix elements $G_{a a}^{s}$ are given by [29]

$$
\begin{aligned}
G_{a a}^{s}= & \pi \sum_{s^{\prime}} \sum_{a^{\prime \prime}} \sum_{b, b^{\prime \prime}} p_{b}^{\tilde{s}} \delta\left(E_{a}^{s}-E_{a^{\prime \prime}}^{s^{\prime}}+E_{b}^{\tilde{s}}-E_{b^{\prime \prime}}^{\tilde{s}^{\prime}}\right) \\
& \times\left\langle a, b, s\left|H_{\mathrm{int}}\right| a^{\prime \prime}, b^{\prime \prime}, s^{\prime}\right\rangle\left\langle a^{\prime \prime}, b^{\prime \prime}, s^{\prime}\left|H_{\mathrm{int}}\right| a, b, s\right\rangle .
\end{aligned}
$$

The commutation relation (92) and the eigenvalue equation (94) imply that the matrix elements associated with the operator $\Delta H_{A}$ are defined as, i.e.,

$$
\Delta H_{a^{\prime} a}^{s} \equiv \delta_{a^{\prime} a}\left\langle a, s\left|\Delta H_{A}\right| a, s\right\rangle .
$$

The matrix elements $\Delta H_{a a}^{s}$ are defined as [29]

$$
\begin{aligned}
\Delta H_{a a}^{s}= & \sum_{s^{\prime}} \sum_{a^{\prime \prime}} \sum_{b, b^{\prime \prime}} p_{b}^{\tilde{s}} \\
& \times \frac{\left\langle a, b, s\left|H_{\text {int }}\right| a^{\prime \prime}, b^{\prime \prime}, s^{\prime}\right\rangle\left\langle a^{\prime \prime}, b^{\prime \prime}, s^{\prime}\left|H_{\text {int }}\right| a, b, s\right\rangle}{E_{a}^{s}-E_{a^{\prime \prime}}^{s^{\prime}}+E_{b}^{\tilde{s}}-E_{b^{\prime \prime}}^{\tilde{s} \prime}}
\end{aligned}
$$

if and only if the energy levels of the system $A$ and of the bath $B$ differ, respectively, i.e., $E_{a^{\prime \prime}}^{s^{\prime}} \neq E_{a}^{s}$ and $E_{b^{\prime \prime}}^{\tilde{s}^{\prime \prime}} \neq E_{b}^{\tilde{s}}$; otherwise they vanish. The matrix elements associated with the superoperator $\Gamma_{s}^{s^{\prime}}\left[\rho_{A}^{s^{\prime}}(t)\right]$ are given by [29]

$$
\left\langle a^{\prime \prime}, s\left|\Gamma_{s}^{s^{\prime}}\left[\rho_{A}^{s^{\prime}}(t)\right]\right| a^{\prime \prime \prime}, s\right\rangle \equiv \sum_{s^{\prime}} \sum_{a, a^{\prime}} \Gamma_{s a^{\prime \prime} a^{\prime \prime \prime}}^{s^{\prime} a^{\prime}} a_{a a^{\prime}}^{s^{\prime}}(t),
$$

where the elements of the reduced density operator $\rho_{A}^{s^{\prime}}(t)$ of the system $A$ are given by

$$
\begin{aligned}
\rho_{a a^{\prime}}^{s^{\prime}}(t)=\rho_{a^{\prime} a}^{s^{\prime}}{ }^{*}(t) & \equiv\left\langle a, s^{\prime}\left|\rho_{A}^{s^{\prime}}(t)\right| a^{\prime}, s^{\prime}\right\rangle \\
& =\sum_{b}\left\langle a, b, s^{\prime}|\rho(t)| a^{\prime}, b, s^{\prime}\right\rangle
\end{aligned}
$$

and the fluctuation and dissipation coefficients $\Gamma_{s^{\prime} a^{\prime \prime} a^{\prime \prime \prime}}^{s a^{\prime}}$ are given by [29]

$$
\begin{aligned}
\Gamma_{s a^{\prime \prime} a^{\prime \prime \prime}}^{s^{\prime} a a^{\prime}}= & \Gamma_{s a^{\prime \prime \prime} a^{\prime \prime}}^{s^{\prime}{ }^{\prime}{ }^{*}} \\
= & \frac{2 \pi}{\hbar} \sum_{b, b^{\prime \prime}} p_{b}^{\tilde{s^{\prime}}} \delta\left(E_{a}^{s^{\prime}}-E_{a^{\prime \prime}}^{s}+E_{b}^{\tilde{s}}-E_{b^{\prime \prime}}^{\tilde{s}}\right) \\
& \times\left\langle a^{\prime}, b, s^{\prime}\left|H_{\mathrm{int}}\right| a^{\prime \prime \prime}, b^{\prime \prime}, s\right\rangle\left\langle a^{\prime \prime}, b^{\prime \prime}, s\left|H_{\mathrm{int}}\right| a, b, s^{\prime}\right\rangle
\end{aligned}
$$

if and only if the transition energies of the system $A$ are equal, i.e., $E_{a}^{s^{\prime}}-E_{a^{\prime \prime}}^{s}=E_{a^{\prime}}^{s^{\prime}}-E_{a^{\prime \prime}}^{s}$; otherwise they vanish. They consist of an imaginary part that describes the fluctuations (i.e., the so-called "Rabi oscillations") and a real part that describes the dissipation (i.e., the transition rates). The matrix elements $H_{\text {ext }_{a a^{\prime}}}^{s}(t)$ of the external Hamiltonian $H_{\text {ext }}(t)$ are written

$$
H_{\text {ext }}^{\stackrel{s}{s} a^{\prime}}(t)=\left\langle s, a\left|H_{\text {ext }}(t)\right| s, a^{\prime}\right\rangle .
$$

The definitions (99) and (104) imply

$$
G_{a a}^{s}=\frac{\hbar}{2} \sum_{s^{\prime}} \sum_{a^{\prime \prime}} \Gamma_{s^{\prime} a^{\prime \prime} a^{\prime \prime}}^{s a} .
$$

Using the relations (98) and (100)-(106), the matrix elements of the master equation (90) are given by

$$
\begin{aligned}
\frac{d}{d t} \rho_{a^{\prime \prime} a^{\prime \prime \prime}}^{s}(t)= & \sum_{s^{\prime}} \sum_{a, a^{\prime}} \Gamma_{s a^{\prime \prime} a^{\prime \prime \prime}}^{s^{\prime} a a^{\prime}} a_{a a^{\prime}}^{s^{\prime}}(t) \\
& -\frac{1}{2} \sum_{s^{\prime}} \sum_{a, a^{\prime}}\left(\Gamma_{s^{\prime} a^{\prime} a^{\prime}}^{s a^{\prime \prime \prime} a^{\prime \prime \prime}}+\Gamma_{s^{\prime} a^{\prime} a^{\prime}}^{s a^{\prime \prime}}\right) \rho_{a^{\prime \prime} a^{\prime \prime \prime}}^{s}(t) \\
& -\frac{i}{\hbar}\left(E_{a^{\prime \prime}}^{s}-E_{a^{\prime \prime \prime}}^{s}+\Delta H_{a^{\prime \prime} a^{\prime \prime}}^{s}-\Delta H_{a^{\prime \prime \prime} a^{\prime \prime \prime}}^{s}\right) \rho_{a^{\prime \prime} a^{\prime \prime \prime}}^{s}(t) \\
& -\frac{i}{\hbar} \sum_{a}\left[H_{\mathrm{ext}_{a^{\prime \prime} a}^{s}(t)}^{s}(t) \rho_{a a^{\prime \prime \prime}}^{s}(t)-H_{\mathrm{ext}_{a a^{\prime \prime \prime}}^{s}}^{s}(t) \rho_{a^{\prime \prime} a}^{s}(t)\right]
\end{aligned}
$$


The energy condition imposed on the fluctuation and dissipation coefficients $\Gamma_{s a^{\prime \prime} a^{\prime \prime \prime}}^{s^{\prime}}$, i.e.,

$$
\Gamma_{s a^{\prime \prime} a^{\prime \prime \prime}}^{s^{\prime} a a^{\prime}} \neq 0 \quad \Leftrightarrow \quad E_{a}^{s^{\prime}}-E_{a^{\prime}}^{s^{\prime}}=E_{a^{\prime \prime}}^{s}-E_{a^{\prime \prime \prime}}^{s} .
$$

The transition rate from the energy level $E_{a}^{s}$ to the energy level $E_{a^{\prime}}^{s^{\prime}}$ are a subset of the fluctuation and dissipation coefficients (108) given by

$$
\begin{aligned}
\Gamma_{a \rightarrow a^{\prime}}^{s \rightarrow s^{\prime}} \equiv & \Gamma_{s^{\prime} a^{\prime} a^{\prime}}^{s a a} \\
= & \frac{2 \pi}{\hbar} \sum_{b, b^{\prime}} p_{b}^{\tilde{s}} \delta\left(E_{a}^{s}-E_{a^{\prime}}^{s^{\prime}}+E_{b}^{\tilde{s}}-E_{b^{\prime}}^{\tilde{s}^{\prime}}\right) \\
& \times\left|\left\langle a^{\prime}, b^{\prime}, s^{\prime}\left|H_{\mathrm{int}}\right| a, b, s\right\rangle\right|^{2},
\end{aligned}
$$

which corresponds to a generalization of Fermi's golden rule between the energy levels $E_{a}^{s}$ and $E_{a^{\prime}}^{s^{\prime}}$, where the statistical equilibrium of the bath $B$ is taken into account via the probability distribution $p_{b}^{\tilde{s}}$. Taking into account the definition (109) of the transition rates and the energy condition (108), the diagonal component of the master equation (107) yields the evolution of the "population" $p_{a}^{s}(t) \equiv \rho_{a a}^{s}(t)$ of the energy level $E_{a}^{s}$, i.e.,

$$
\begin{aligned}
\frac{d}{d t} p_{a}^{s}(t)= & \sum_{s^{\prime}} \sum_{a^{\prime}}\left[\Gamma_{a^{\prime} \rightarrow a}^{s^{\prime} \rightarrow s} p_{a^{\prime}}^{s^{\prime}}(t)-\Gamma_{a \rightarrow a^{\prime}}^{s \rightarrow s^{\prime}} p_{a}^{s}(t)\right] \\
& -\frac{i}{\hbar} \sum_{a^{\prime}}\left[H_{\operatorname{ext}_{a a^{\prime}}}^{s}(t) \rho_{a^{\prime} a}^{s}(t)-H_{\operatorname{ext}_{a^{\prime} a}}^{s}(t) \rho_{a a^{\prime}}^{s}(t)\right] .
\end{aligned}
$$

Note that according to the relation (109), the transition rates $\Gamma_{a^{\prime} \rightarrow a}^{s^{\prime} \rightarrow s}$ and $\Gamma_{a \rightarrow a^{\prime}}^{s \rightarrow s^{\prime}}$ are real and positive definite.

The definition (109) of the transition rates and the energy condition (108) imply that the evolution equation (107) for the "coherences" $\rho_{a a^{\prime}}^{s}(t)$ is given by

$$
\begin{aligned}
& \frac{d}{d t} \rho_{a a^{\prime}}^{s}(t) \\
& =\left[\Gamma_{s a a^{\prime}}^{s a a^{\prime}}-\frac{1}{2} \sum_{s^{\prime}} \sum_{a^{\prime \prime}}\left(\Gamma_{a \rightarrow a^{\prime \prime}}^{s \rightarrow s^{\prime}}+\Gamma_{\begin{array}{c}
s \rightarrow s^{\prime} \\
a^{\prime} \rightarrow a^{\prime \prime}
\end{array}}\right)\right] \rho_{a a^{\prime}}^{s}(t) \\
& -\frac{i}{\hbar}\left(E_{a}^{s}-E_{a^{\prime}}^{s}\right) \rho_{a a^{\prime}}^{s}(t)+\sum_{s^{\prime}} \sum_{a^{\prime \prime}, a^{\prime \prime \prime}} \Gamma_{s a a^{\prime}}^{s^{\prime} a^{\prime \prime} a^{\prime \prime \prime}} \rho_{a^{\prime \prime} a^{\prime \prime \prime}}^{s^{\prime}}(t) \\
& -\frac{i}{\hbar}\left(E_{a}^{s}-E_{a^{\prime}}^{s}+\Delta H_{a a}^{s}-\Delta H_{a^{\prime} a^{\prime}}^{s}\right) \rho_{a a^{\prime}}^{s}(t) \\
& -\frac{i}{\hbar} \sum_{a^{\prime \prime}}\left[H_{\operatorname{ext}_{a a^{\prime \prime}}}^{s}(t) \rho_{a^{\prime \prime} a^{\prime}}^{s}(t)-H_{\operatorname{ext}_{a^{\prime \prime} a^{\prime}}}^{s}(t) \rho_{a a^{\prime \prime}}^{s}(t)\right] \text {. }
\end{aligned}
$$

\section{RELAXATION OF A VIBRATIONAL, ROTATIONAL, AND SPIN SYSTEM}

This section is devoted to the description of dissipative molecular processes, where the vibrational and rotational molecular properties and the electronic spin properties are coupled to a bath and to an external magnetic field. We consider a molecule interacting with an external magnetic field where the characteristic time scale associated with the electronic orbital dynamics is sufficiently small compared to the characteristic time scales of the electronic spin dynamics and of the vibrational and rotational molecular dynamics. The system $A$ has vibrational and rotational molecular properties and electronic spin properties, and the bath $B$ has electronic spatial properties. The system $A$ and the bath $B$ are assumed to be weakly coupled and weakly correlated. In a first approach, we do not take into account the dynamical contribution due to the nuclear spins and consider only the electronic spins.

\section{A. Hamiltonians}

We briefly review the main results of Ref. [30]. In order to obtain an explicit expression for the Hamiltonian of the system $H_{A}$, the Hamiltonian of the bath $H_{B}$ and the interaction Hamiltonian $H_{\text {int }}$, it is necessary to separate the properties associated with system $A$ from the properties associated with the bath $B$. The properties associated with system $A$ are functions of the molecular deformation amplitudes and momenta $Q_{\alpha}$ and $P_{\alpha}$ associated with the deformation eigenmodes $\alpha$ of the molecular orbital angular momentum $\boldsymbol{L}(\boldsymbol{\omega})$ and of the spin $\boldsymbol{s}_{v}(\boldsymbol{\omega})$ of the electron $v$. The properties associated with the bath $B$ that are function of the electronic positions and momenta $\boldsymbol{q}_{v}$ and $\boldsymbol{p}_{v}$. We consider that the center of mass of the system is fixed as usual.

To first order, the molecular inertia tensor is given by

$$
\mathrm{I}(Q .)=\mathrm{I}_{0} \mathbb{1}+\sum_{\alpha=1}^{3 N-6} \mathrm{I}_{\alpha} Q_{\alpha}+\mathcal{O}(2),
$$

where $I_{0}$ is the equilibrium molecular inertia tensor. The inverse of the molecular inertia tensor is given by

$$
\mathrm{I}(Q .)^{-1}=\mathrm{I}_{0}^{-1} \mathbb{1}+\sum_{\alpha=1}^{3 N-6} \tilde{\mathrm{I}}_{\alpha}^{-1} Q_{\alpha}+\mathcal{O}(2),
$$

where $\tilde{\mathrm{I}}_{\alpha}^{-1}=\mathrm{I}_{0}^{-1} \cdot \mathrm{I}_{\alpha} \cdot \mathrm{I}_{0}^{-1}$. The spatial symmetries of the inertia tensor are expressed in terms of the molecular orientation operator $\omega \in \mathcal{L}\left(\mathcal{H}_{\text {ori }}\right)$. We introduce explicitly the operators $\boldsymbol{e}_{k}(\boldsymbol{\omega})$ and $\boldsymbol{e}^{k}(\boldsymbol{\omega})$ that are obtained by performing a molecular rotation described by the operator $R(\omega)$ on the basis vectors $\boldsymbol{e}_{j}$ and $\boldsymbol{e}^{j}$, i.e.,

$$
\begin{aligned}
& \boldsymbol{e}_{k}(\boldsymbol{\omega})=\left[\boldsymbol{e}^{j} \cdot \mathrm{R}(\boldsymbol{\omega}) \cdot \boldsymbol{e}_{k}\right] \boldsymbol{e}_{j}, \\
& \boldsymbol{e}^{k}(\boldsymbol{\omega})=\left[\boldsymbol{e}^{k} \cdot \mathrm{R}(\boldsymbol{\omega}) \cdot \boldsymbol{e}_{j}\right] \boldsymbol{e}^{j},
\end{aligned}
$$

where we used the Einstein summation convention for the spatial indices $j=1, \ldots, 3$. The operators $\boldsymbol{e}_{k}(\boldsymbol{\omega})$ and $\boldsymbol{e}^{k}(\boldsymbol{\omega})$ belong to the fiber bundle $\mathbb{R}^{3} \times \mathcal{L}\left(\mathcal{H}_{\text {ori }}\right)$, where the base space is $\mathbb{R}^{3}$ and the fiber is $\mathcal{L}\left(\mathcal{H}_{\text {ori }}\right)$. The basis vectors $\boldsymbol{e}_{k}$ and $\boldsymbol{e}^{k}$ belong to the base space $\mathbb{R}^{3}$ and the molecular orientation operator $\omega$ belongs to the fiber $\mathcal{L}\left(\mathcal{H}_{\text {ori }}\right)$. For given basis vector sets $\left\{\boldsymbol{e}_{j}\right\}$ and $\left\{\boldsymbol{e}^{j}\right\}$, the rotation operator $\mathrm{R}(\boldsymbol{\omega})$ is chosen such that the equilibrium molecular inertia tensor $I_{0}$ is diagonal with respect to the orthonormal operator basis $\left\{\boldsymbol{e}^{k}(\boldsymbol{\omega})\right\}$, which implies that the inverse equilibrium molecular inertia tensor $I_{0}^{-1}$ is diagonal, too. The free Hamiltonians $H_{A}$ and $H_{B}$ containing 
the dominant dynamical contributions are defined as

$$
\begin{aligned}
H_{A}= & \sum_{\alpha=1}^{3 N-6}\left(\frac{P_{\alpha}^{2}}{2}+\frac{1}{2} \omega_{\alpha}^{2} Q_{\alpha}^{2}\right)+\frac{1}{2} \boldsymbol{L}(\boldsymbol{\omega}) \cdot \mathrm{I}_{0}^{-1} \cdot \boldsymbol{L}(\boldsymbol{\omega}) \\
& -\boldsymbol{L}(\boldsymbol{\omega}) \cdot\left\langle\boldsymbol{\Omega}\left(\boldsymbol{q} ., \boldsymbol{p}_{.}\right)\right\rangle-\gamma_{e} \sum_{\nu=1}^{n} \boldsymbol{s}_{v}(\boldsymbol{\omega}) \cdot\left\langle\boldsymbol{B}_{v}\left(\boldsymbol{q}_{.}, \boldsymbol{p}_{\nu}\right)\right\rangle \\
H_{B}= & \sum_{\nu=1}^{n} \frac{\boldsymbol{p}_{v}^{2}}{2 m}+\frac{1}{2} \ell\left(\boldsymbol{q} ., \boldsymbol{p}_{.}\right) \cdot \mathrm{I}_{0}^{-1} \cdot \boldsymbol{\ell}(\boldsymbol{q} ., \boldsymbol{p} .)+V(\boldsymbol{q} .),
\end{aligned}
$$

where $\omega_{\alpha}$ is the angular frequency of the vibration eigenmode $\alpha, \boldsymbol{\ell}(\boldsymbol{q}, \boldsymbol{p}$. $)$ is the electronic orbital angular momentum and $\boldsymbol{\omega}$ is the molecular orientation operator. The molecular angular velocity operator $\boldsymbol{\Omega}(\boldsymbol{q}, \boldsymbol{p}$.) is given by

$$
\boldsymbol{\Omega}\left(\boldsymbol{q}_{.}, \boldsymbol{p}_{.}\right)=\mathrm{I}_{0}^{-1} \cdot \boldsymbol{\ell}\left(\boldsymbol{q}_{.}, \boldsymbol{p}_{.}\right) .
$$

The magnetic induction field operator $\boldsymbol{B}_{v 0}\left(\boldsymbol{q}_{.}, \boldsymbol{p}_{v}\right)$ generated by the relative motion of the nuclei and the other electrons with respect to the electron $v$ reads

$$
\boldsymbol{B}_{v}\left(\boldsymbol{q}_{.}, \boldsymbol{p}_{v}\right)=\frac{1}{m c^{2}}\left[\boldsymbol{E}_{v 0}(\boldsymbol{q} .) \times \boldsymbol{p}_{v}\right]
$$

where the electric field $\boldsymbol{E}_{\nu 0}(\boldsymbol{q}$.) is the lowest-order term of the series expansion of the electric field $\boldsymbol{E}_{v}(Q ., q$.$) exerted$ on the electron $v$ in terms of the deformation operator $Q_{\alpha}$, i.e.,

$$
\boldsymbol{E}_{\nu}\left(Q_{.}, \boldsymbol{q} .\right)=\boldsymbol{E}_{\nu 0}(\boldsymbol{q} .)+\boldsymbol{E}_{\nu \alpha}(\boldsymbol{q} .) Q_{\alpha}+\mathcal{O}(2) .
$$

The last two terms of the expression (115) of the Hamiltonian $H_{A}$ are polarization terms where the properties of the bath have been averaged out. There is no polarization term associated with the Coulomb potential due to the molecular equilibrium condition established explicitly in Ref. [30]. The Coulomb potential $V(\boldsymbol{q}$. $)$ to zeroth-order in the deformation operator $Q_{\alpha}$ is the sum of the electronic repulsion potential $V_{e-e}(\boldsymbol{q}$. and the of the attraction potential $V_{N-e(0)}(\boldsymbol{q}$.) in the molecular equilibrium configuration,

$$
V(\boldsymbol{q} .)=V_{e-e}(\boldsymbol{q} .)+V_{N-e(0)}(\boldsymbol{q} .) .
$$

The interaction Hamiltonian $H_{\text {int }}$ consists of three different parts: a deformation part $H_{\text {def }}$ describing the molecular vibrations, an orientation part $H_{\text {ori }}$ describing the molecular rotations, and a "spin-orbit" part $H_{\mathrm{SO}}$ describing the dynamics of the electronic spins, i.e.,

$$
H_{\text {int }}=H_{\text {def }}+H_{\text {ori }}+H_{\text {SO }}
$$

to follow the convention used in atomic physics, which are given to first order by

$$
\begin{aligned}
& H_{\mathrm{def}}=\sum_{\alpha=1}^{3 N-6} Q_{\alpha} \otimes \tilde{V}_{N-e(\alpha)}(\boldsymbol{q} .) \\
& H_{\mathrm{ori}}=-\boldsymbol{e}_{k}(\boldsymbol{\omega}) \cdot \boldsymbol{L}(\boldsymbol{\omega}) \otimes \boldsymbol{e}^{k}(\boldsymbol{\omega}) \cdot \tilde{\boldsymbol{\Omega}}\left(\boldsymbol{q} ., \boldsymbol{p}_{.}\right) \\
& H_{\mathrm{SO}}=-\gamma_{e} \sum_{\nu=1}^{n} \boldsymbol{e}_{k}(\boldsymbol{\omega}) \cdot \boldsymbol{s}_{\nu}(\boldsymbol{\omega}) \otimes \boldsymbol{e}^{k}(\boldsymbol{\omega}) \cdot \tilde{\boldsymbol{B}}_{\nu}\left(\boldsymbol{q} ., \boldsymbol{p}_{v}\right),
\end{aligned}
$$

where the operators acting on the Hilbert space of the bath are defined as

$$
\begin{aligned}
\tilde{V}_{N-e(\alpha)}(\boldsymbol{q} .) & =V_{N-e(\alpha)}(\boldsymbol{q} .)-\left\langle V_{N-e(\alpha)}(\boldsymbol{q} .)\right\rangle \mathbb{1} \\
\tilde{\boldsymbol{\Omega}}\left(\boldsymbol{q}_{.}, \boldsymbol{p}_{.}\right) & =\boldsymbol{\Omega}\left(\boldsymbol{q}_{.}, \boldsymbol{p}_{.}\right)-\left\langle\boldsymbol{\Omega}\left(\boldsymbol{q}_{.}, \boldsymbol{p}_{.}\right)\right\rangle \mathbb{1} \\
\tilde{\boldsymbol{B}}_{v}\left(\boldsymbol{q}_{.}, \boldsymbol{p}_{v}\right) & =\boldsymbol{B}_{v}\left(\boldsymbol{q}_{.}, \boldsymbol{p}_{v}\right)-\left\langle\boldsymbol{B}_{v}\left(\boldsymbol{q}_{.}, \boldsymbol{p}_{v}\right)\right\rangle \mathbb{1} .
\end{aligned}
$$

Here we use the fact that the polarization terms do not play any dynamical role and can be subtracted. The external Hamiltonian $H_{\text {ext }}(t)$ consists of a spatial part and a spin part, i.e.,

$$
H_{\mathrm{ext}}(t)=-\gamma \boldsymbol{L}(\boldsymbol{\omega}) \cdot \boldsymbol{B}_{\mathrm{ext}}(\boldsymbol{\omega}, t)-\gamma_{e} \sum_{\nu=1}^{n} \boldsymbol{s}_{\nu}(\boldsymbol{\omega}) \cdot \boldsymbol{B}_{\mathrm{ext}}(\boldsymbol{\omega}, t),
$$

where $\gamma$ is the effective molecular magnetic ratio and $\boldsymbol{B}_{\text {ext }}(\boldsymbol{\omega}, t)$ is the rotated external magnetic field operator. The rotated spin operator $\boldsymbol{s}_{v}(\boldsymbol{\omega})$ and the rotated external magnetic field $\boldsymbol{B}_{\text {ext }}(\boldsymbol{\omega}, t)$ are pseudovectorial operators that are functions of the molecular orientation operator $\boldsymbol{\omega}$. They are respectively related to the spin operator $\boldsymbol{s}_{v}$ and the external magnetic field $\boldsymbol{B}_{\text {ext }}(t)$ in the following manner:

$$
\begin{array}{r}
\boldsymbol{s}_{v}(\boldsymbol{\omega})=\left(\hat{\boldsymbol{\omega}} \cdot \boldsymbol{s}_{v}\right) \hat{\boldsymbol{\omega}}+\left[\boldsymbol{s}_{v}-\left(\hat{\boldsymbol{\omega}} \cdot \boldsymbol{s}_{v}\right) \hat{\boldsymbol{\omega}}\right] \cos \omega-\left(\hat{\boldsymbol{\omega}} \times \boldsymbol{s}_{v}\right) \sin \omega, \\
\boldsymbol{B}_{\mathrm{ext}}(\boldsymbol{\omega}, t)=\left[\hat{\boldsymbol{\omega}} \cdot \boldsymbol{B}_{\mathrm{ext}}(t)\right] \hat{\boldsymbol{\omega}}+\left\{\boldsymbol{B}_{\mathrm{ext}}(t)-\left[\hat{\boldsymbol{\omega}} \cdot \boldsymbol{B}_{\mathrm{ext}}(t)\right] \hat{\boldsymbol{\omega}}\right\} \cos \omega \\
-\left[\hat{\boldsymbol{\omega}} \times \boldsymbol{B}_{\mathrm{ext}}(t)\right] \sin \omega,
\end{array}
$$

where $\omega=\sqrt{\boldsymbol{\omega} \cdot \boldsymbol{\omega}}$ and $\hat{\boldsymbol{\omega}}=\boldsymbol{\omega} / \omega$. The rotational identities (127) and (128) generalize Rodrigues' rotation formula for vectorial operators. The transformation law for the spin operator (127) is established explicitly in Appendix A, where the operators $\boldsymbol{s}_{v}$ and $\boldsymbol{s}_{v}(\boldsymbol{\omega})$ are treated as dimensionless operators. The external magnetic field $\boldsymbol{B}_{\text {ext }}(t)$ is a classical field, but the rotated external magnetic field $\boldsymbol{B}_{\text {ext }}(\boldsymbol{\omega}, t)$ is a field operator since it is a function of the orientation operator $\boldsymbol{\omega}$.

\section{B. Interaction Hamiltonian}

According to the relation (121), the interaction Hamiltonian $H_{\text {int }}$ consists of the deformation Hamiltonian $H_{\text {def }}$, the orientation Hamiltonian $H_{\text {ori }}$, and the spin-orbit Hamiltonian $H_{\text {SO }}$. We shall now express these Hamiltonians as direct sums of block operators [29].

Since the molecular deformation operator $Q_{\alpha}$ is an observable of the system $A$ and the operator

$$
\tilde{V}_{\alpha} \equiv \tilde{V}_{N-e(\alpha)}(\boldsymbol{q} .)
$$

is an observable of the bath $B$, they commute with the projectors $\mathcal{P}_{A}^{s}$ and $\mathcal{P}_{B}^{\tilde{s}}$, respectively, according to the commutation relations (63). These operators can be decomposed into square block operators according to

$$
\begin{gathered}
Q_{\alpha}=\left.\bigoplus_{s} Q_{\alpha}^{s} \equiv \bigoplus_{s} \mathcal{P}_{A}^{s} Q_{\alpha} \mathcal{P}_{A}^{s}\right|_{\mathcal{H}_{A}^{s} \rightarrow \mathcal{H}_{A}^{s}}, \\
\tilde{V}_{\alpha}=\left.\bigoplus_{\tilde{s}} \tilde{V}_{\alpha}^{\tilde{s}} \equiv \bigoplus_{\tilde{s}} \mathcal{P}_{B}^{\tilde{s}} \tilde{V}_{\alpha} \mathcal{P}_{B}^{\tilde{s}}\right|_{\mathcal{H}_{B}^{\tilde{s}} \rightarrow \mathcal{H}_{B}^{\tilde{s}}} .
\end{gathered}
$$


The substitution of the decompositions (130) into expression (122) yields

$$
H_{\mathrm{def}}=\bigoplus_{s} \sum_{\alpha=1}^{3 N-6} Q_{\alpha}^{s} \otimes \tilde{V}_{\alpha}^{\tilde{s}} .
$$

Since the spatial $k$ component of the molecular orbital angular momentum operator,

$$
L_{k} \equiv \boldsymbol{e}_{k}(\boldsymbol{\omega}) \cdot \boldsymbol{L}(\boldsymbol{\omega}),
$$

is an observable of the system $A$ and the spatial $k$ component of the molecular angular velocity operator

$$
\tilde{\Omega}^{k} \equiv \boldsymbol{e}^{k}(\boldsymbol{\omega}) \cdot \tilde{\boldsymbol{\Omega}}(\boldsymbol{q} ., \boldsymbol{p} .)
$$

is an observable of the bath $B$, they commute with the projectors $\mathcal{P}_{A}^{s}$ and $\mathcal{P}_{B}^{\tilde{s}}$, respectively, according to the commutation relations (63). These operators can be decomposed into square block operators according to

$$
\begin{aligned}
& L_{k}=\left.\bigoplus_{s} L_{k}^{s} \equiv \bigoplus_{s} \mathcal{P}_{A}^{s} L_{k} \mathcal{P}_{A}^{s}\right|_{\mathcal{H}_{A}^{s} \rightarrow \mathcal{H}_{A}^{s}} \\
& \tilde{\Omega}_{k}=\left.\bigoplus_{\tilde{s}} \tilde{\Omega}^{\tilde{s} k} \equiv \bigoplus_{\tilde{s}} \mathcal{P}_{B}^{\tilde{s}} \tilde{\Omega}_{k} \mathcal{P}_{B}^{\tilde{s}}\right|_{\mathcal{H}_{B}^{\tilde{s}} \rightarrow \mathcal{H}_{B}^{\tilde{s}}}
\end{aligned}
$$

Using the Einstein summation convention for the spatial indices $k=1, \ldots, 3$, the substitution of the decompositions (134) into the expression (123) yields

$$
H_{\text {ori }}=-\bigoplus_{s} L_{k}^{s} \otimes \tilde{\Omega}^{\tilde{s} k}
$$

The spatial $k$ component of the spin operator,

$$
s_{\nu k} \equiv \boldsymbol{e}_{k}(\boldsymbol{\omega}) \cdot \boldsymbol{s}_{\nu}(\boldsymbol{\omega}),
$$

is not an observable of the system $A$ and the spatial $k$ component of the magnetic induction field operator,

$$
\tilde{B}_{v}^{k} \equiv \boldsymbol{e}^{k}(\boldsymbol{\omega}) \cdot \tilde{\boldsymbol{B}}_{v}\left(\boldsymbol{q} ., \boldsymbol{p}_{v}\right),
$$

is not an observable of the system $B$ since they do not commute with the unitary representations $U_{A}(\sigma)$ and $U_{B}(\sigma)$ of the permutation group $S_{n}$, respectively. The actions of the permutation group $S_{n}$ on the spin operator $s_{v k}$ and the operator $\tilde{B}_{v}^{k}$ are given, respectively, by

$$
\begin{aligned}
& U_{A}(\sigma) s_{v k} U_{A}(\sigma)^{-1}=s_{\sigma(v) k}, \\
& U_{B}(\sigma) \tilde{B}_{\nu}^{k} U_{B}(\sigma)^{-1}=\tilde{B}_{\sigma(v)}^{k} .
\end{aligned}
$$

In order to account for the fact that the spin-orbit Hamiltonian $H_{\text {SO }}$ is an observable of the molecule, we decompose the spin operator $s_{v k}$ into block operators that belong to the space of linear operators $\mathcal{L}\left(\mathcal{H}_{A}\right)^{\mu}$ and the magnetic induction field operator $\tilde{B}_{v}^{k}$ into block operators that belong to the space of linear operators $\mathcal{L}\left(\mathcal{H}_{B}\right)^{\mu}$. The spin operator $s_{v k}^{\mu} \in \mathcal{L}\left(\mathcal{H}_{A}\right)^{\mu}$ and the magnetic induction field operator $\tilde{B}_{v}^{\mu k} \in \mathcal{L}\left(\mathcal{H}_{B}\right)^{\mu}$ are defined, respectively, as [29]

$$
\begin{aligned}
s_{v k}^{\mu} & =\mathscr{P}_{A}^{\mu} s_{v k}, \\
\tilde{B}_{v}^{\mu k} & =\mathscr{P}_{B}^{\mu} \tilde{B}_{v}^{\mu k} .
\end{aligned}
$$

Taking into account the decomposition (70) and the expressions (140) and (141), the spin-orbit Hamiltonian (124) is recast as

$$
H_{\mathrm{SO}}=-\gamma_{e} \bigoplus_{\mu} \sum_{\nu=1}^{n} s_{\nu k}^{\mu} \otimes \tilde{B}_{\nu}^{\mu k},
$$

where we used the Einstein summation convention again. Using the relations (138) and (139) and the definition (76) of the orthogonal projectors $\mathscr{P}_{A}^{\mu}$ and $\mathscr{P}_{B}^{\mu}$, the spin operator (140) and the operator (141) can be written explicitly as

$$
\begin{aligned}
& s_{v k}^{\mu}=\frac{d_{\mu}}{n !} \sum_{\sigma \in S_{n}} \chi_{\mu}(\sigma) s_{\sigma(v) k} \equiv \sum_{v^{\prime}=1}^{n} a_{\nu}^{\mu v^{\prime}} s_{\nu^{\prime} k}, \\
& \tilde{B}_{v}^{\mu k}=\frac{d_{\mu}}{n !} \sum_{\sigma \in S_{n}} \chi_{\mu}(\sigma) \tilde{B}_{\sigma(v)}^{k} \equiv \sum_{\nu^{\prime}=1}^{n} a_{v}^{\mu v^{\prime}} \tilde{B}_{v^{\prime}}^{k},
\end{aligned}
$$

where $v^{\prime}=\sigma(v)$ and $a_{v}^{\mu v^{\prime}} \in \mathbb{R}$ since $\chi_{\mu}(\sigma)=\chi_{\mu}(\sigma)^{*} \in \mathbb{R}$. As established explicitly in Ref. [29], the coefficients $a_{v}^{\mu v^{\prime}}$ are given by

$$
\begin{aligned}
a_{v}^{\mu v^{\prime}} & =\frac{d_{\mu}}{n !} \sum_{\sigma \in S_{n} \mid \sigma(v)=v^{\prime}} \chi_{\mu}(\sigma) \\
& =\frac{1}{n} \delta^{\mu[n]}+\left(\delta_{v}^{\nu^{\prime}}-\frac{1}{n}\right) \delta^{\mu[n-1,1]} .
\end{aligned}
$$

According to the relation (143) and (145), the only nonvanishing spin operators $s_{v k}^{\mu}$ are

$$
\begin{aligned}
s_{\nu k}^{[n]} & =\frac{1}{n} \sum_{\nu^{\prime}=1}^{n} s_{\nu^{\prime} k} \equiv \frac{1}{n} s_{k}^{[n]}, \\
s_{\nu k}^{[n-1,1]} & =s_{\nu k}-\frac{1}{n} \sum_{\nu^{\prime}=1}^{n} s_{\nu^{\prime} k},
\end{aligned}
$$

where $s_{k}^{[n]}$ is an observable of the system $A$ since it is invariant under the action of the permutation group $S_{n}$, i.e.,

$$
U_{A}(\sigma) s_{k}^{[n]} U_{A}(\sigma)^{-1}=s_{k}^{[n]} .
$$

Similarly, according to the relation (144) and (145), the only nonvanishing operators $\tilde{B}_{v}^{\mu k}$ are

$$
\begin{aligned}
\tilde{B}_{v}^{[n] k} & =\frac{1}{n} \sum_{v^{\prime}=1}^{n} \tilde{B}_{v^{\prime}}^{k} \equiv \tilde{B}^{[n] k}, \\
\tilde{B}_{v}^{[n-1,1] k} & =\tilde{B}_{v}^{k}-\frac{1}{n} \sum_{v^{\prime}=1}^{n} \tilde{B}_{v^{\prime}}^{k},
\end{aligned}
$$

where $\tilde{B}^{[n] k}$ is an observable of the bath $B$ since it is invariant under the action of the permutation group $S_{n}$, i.e.,

$$
U_{B}(\sigma) \tilde{B}^{[n] k} U_{B}(\sigma)^{-1}=\tilde{B}^{[n] k} .
$$

In view of the relation (145) there are only two types of nonvanishing irreducible representations of the permutation group $S_{n}$, namely $D^{[n]}$ and $D^{[n-1,1]}$, which implies that the spin-orbit Hamiltonian (142) can be expressed as a direct sum according to

$$
H_{\mathrm{SO}}=H_{\mathrm{SO}}^{[n]} \bigoplus H_{\mathrm{SO}}^{[n-1,1]}
$$


where

$$
\begin{aligned}
H_{\mathrm{SO}}^{[n]} & =-\gamma_{e} s_{k}^{[n]} \otimes \tilde{B}^{[n] k}, \\
H_{\mathrm{SO}}^{[n-1,1]} & =-\gamma_{e} \sum_{\nu=1}^{n} s_{\nu k}^{[n-1,1]} \otimes \tilde{B}_{v}^{[n-1,1] k} .
\end{aligned}
$$

According to the relation (70), the spin-orbit Hamiltonian $H_{\mathrm{SO}}^{[n-1,1]}$ has the decomposition

$$
H_{\mathrm{SO}}^{[n-1,1]}=-\sum_{j=1}^{n-1} s_{k j}^{[n-1,1]} \otimes \tilde{B}_{j}^{[n-1,1] k},
$$

where $d_{[n-1,1]}=n-1$ and $a=b \equiv k=1,2,3$. Taking into account the relation (72), the action of the permutation group $S_{n}$ on the spin operator $s_{k j}^{[n-1,1]}$ and on the operator $\tilde{B}_{j}^{[n-1,1] k}$ is given by

$$
\begin{aligned}
U_{A}(\sigma) s_{k j}^{[n-1,1]} U_{A}(\sigma)^{-1} & =\sum_{i=1}^{d_{\mu}} s_{k i}^{[n-1,1]} d_{i j}^{[n-1,1]}(\sigma), \\
U_{B}(\sigma) \tilde{B}_{j}^{[n-1,1] k} U_{B}(\sigma)^{-1} & =\sum_{i=1}^{d_{\mu}} \tilde{B}_{i}^{[n-1,1] k} d_{i j}^{[n-1,1]}(\sigma) .
\end{aligned}
$$

In order to formally identify the expressions (152) and (153), we recast the spin operator $s_{k j}^{[n-1,1]}$ in terms of the spin operator $s_{\nu k}$ and the magnetic induction field operator $\tilde{B}_{j}^{[n-1,1] k}$ in terms of the magnetic induction field operator $\tilde{B}_{v}^{k}$ according to

$$
\begin{aligned}
s_{k j}^{[n-1,1]} & =\sqrt{n} \sum_{\nu=1}^{n} a_{j}^{v} s_{v k}, \\
\tilde{B}_{j}^{[n-1,1] k} & =\frac{1}{\sqrt{n}} \sum_{\nu=1}^{n} a_{j}^{v} \tilde{B}_{v}^{k},
\end{aligned}
$$

where $a_{j}^{v} \in \mathbb{R}$. The coefficients $a_{j}^{v} \in \mathbb{R}$ define $n-1$ vectors $\left|a_{j}\right\rangle \equiv\left(a_{j}^{1}, \ldots, a_{j}^{n}\right)$, that are orthogonal to the vector $\left|a_{n}\right\rangle \equiv$ $(1, \ldots, 1)$ and span the Hilbert space $\mathcal{H}_{A}^{[n-1,1]}$, i.e.,

$$
\left\langle a_{n} \mid a_{j}\right\rangle=0 \quad \text { and } \quad\left\langle a_{j} \mid a_{\ell}\right\rangle=\delta_{j \ell},
$$

which is expressed explicitly in terms of the coefficients $a_{j}^{v}$ as

$$
\sum_{\nu=1}^{n} a_{j}^{\nu}=0 \quad \text { and } \quad \sum_{\nu=1}^{n} a_{j}^{v} a_{\ell}^{v}=\delta_{j \ell} .
$$

Taking into account the relations (146), (148), and (155), the coefficients $a_{j}^{v}$ have to satisfy the identity

$$
\sum_{j=1}^{n-1} a_{j}^{v} a_{j}^{\nu^{\prime}}=\delta^{\nu \nu^{\prime}}-\frac{1}{n}
$$

since the expressions (152) and (153) are equal. Relations (157) and (158) do not uniquely determine the coefficients $a_{j}^{v}$. The coefficients with the highest symmetry are given by

$$
\begin{aligned}
& a_{j}^{v}=-\delta_{j}^{v}+\frac{1+\sqrt{n}}{(n-1) \sqrt{n}} \quad \text { if } v=1, \ldots, n-1, \\
& a_{j}^{n}=-\frac{1}{\sqrt{n}} .
\end{aligned}
$$

The substitution of the relations (146) and (148) into the expressions (154) taking into account the relations (138) and (139) yields

$$
d_{j \ell}^{[n-1,1]}(\sigma)=\sum_{\nu=1}^{n} a_{j}^{\sigma(v)} a_{\ell}^{\nu}
$$

The spin operator $s_{k}^{[n]}$ and the magnetic induction field operator $\tilde{B}^{[n] k}$ can be decomposed into square blocks operators according to

$$
\begin{aligned}
s_{k}^{[n]} & =\left.\bigoplus_{\substack{s, s^{\prime} \\
s^{\prime}=s}} s_{k}^{s^{\prime}[n] s} \equiv \bigoplus_{\substack{s, s^{\prime} \\
s^{\prime}=s}} \mathcal{P}_{A}^{s^{\prime}} s_{k}^{[n]} \mathcal{P}_{A}^{s}\right|_{\mathcal{H}_{A}^{s} \rightarrow \mathcal{H}_{A}^{s^{\prime}}}, \\
\tilde{B}^{[n] k} & =\left.\bigoplus_{\substack{\tilde{s}, \tilde{s}^{\prime} \\
\tilde{s}=\tilde{s}}} \tilde{B}^{s^{\prime}[n] s k} \equiv \bigoplus_{\substack{\tilde{s}, \tilde{s}^{\prime} \\
\tilde{s}=\tilde{s}^{\prime}}} \mathcal{P}_{B}^{\tilde{s}^{\prime}} \tilde{B}^{[n] k} \mathcal{P}_{B}^{\tilde{s}}\right|_{\mathcal{H}_{B}^{\tilde{s}} \rightarrow \mathcal{H}_{B}^{\tilde{s}}} \cdot
\end{aligned}
$$

The spin operator $s_{k j}^{[n-1,1]}$ and the magnetic induction field operator $\tilde{B}_{j}^{[n-1,1] k}$ can be decomposed into strictly rectangular blocks operators according to

$$
\begin{aligned}
& s_{k j}^{[n-1,1]}=\left.\bigoplus_{\substack{s, s^{\prime} \\
s^{\prime} \neq s}} s_{k}^{s^{\prime}[n-1,1] s} \equiv \bigoplus_{\substack{s, s^{\prime} \\
s^{\prime} \neq s}} \mathcal{P}_{A}^{s^{\prime}} s_{k j}^{[n-1,1]} \mathcal{P}_{A}^{s}\right|_{\mathcal{H}_{A}^{s} \rightarrow \mathcal{H}_{A}^{s^{\prime}}}, \\
& \tilde{B}_{j}^{[n-1,1] k}=\left.\bigoplus_{\substack{\tilde{s}, \tilde{s}^{\prime} \\
\tilde{s} \neq \tilde{s}^{\prime}}} \tilde{B}_{j}^{\tilde{s}^{\prime}[n-1,1] \tilde{s} k} \equiv \bigoplus_{\substack{\tilde{s}, \tilde{s}^{\prime} \\
\tilde{s} \neq \tilde{s}^{\prime}}} \mathcal{P}_{B}^{\tilde{s}^{\prime}} \tilde{B}_{j}^{[n-1,1] k} \mathcal{P}_{B}^{\tilde{s}}\right|_{\mathcal{H}_{B}^{\tilde{s}} \rightarrow \mathcal{H}_{B}^{s^{\prime}}} \cdot
\end{aligned}
$$

The substitution of the decompositions (161) and (162) into the expressions (151) yields

$$
\begin{gathered}
H_{\mathrm{SO}}^{[n]}=-\gamma_{e} \bigoplus_{\substack{s, s^{\prime} \\
s^{\prime}=s}} s_{k}^{s^{\prime}[n] s} \otimes \tilde{B}^{\tilde{s}^{\prime}[n] \tilde{s} k}, \\
H_{\mathrm{SO}}^{[n-1,1]}=-\gamma_{e} \bigoplus_{\substack{s, s^{\prime} \\
s^{\prime} \neq s}} \sum_{j=1}^{n-1} s_{k j}^{s^{\prime}[n-1,1] s} \otimes \tilde{B}_{j}^{\tilde{s}^{\prime}[n-1,1] \tilde{s} k} .
\end{gathered}
$$

The operator $H_{\mathrm{SO}}^{[n]}$ generates transitions that do not change the total electronic spin of the system $A$ and the operator $H_{\mathrm{SO}}^{[n-1,1]}$ generates transitions that change it.

\section{External Hamiltonian}

The operators $\Omega_{\mathrm{ext}}^{k}(t)$ and $B_{\mathrm{ext}}^{k}(t)$ are defined in terms of the external field $\boldsymbol{B}_{\text {ext }}(\boldsymbol{\omega}, t)$ as

$$
\begin{aligned}
\Omega_{\mathrm{ext}}^{k}(t) & \equiv \gamma \boldsymbol{e}^{k}(\boldsymbol{\omega}) \cdot \boldsymbol{B}_{\mathrm{ext}}(\boldsymbol{\omega}, t), \\
B_{\mathrm{ext}}^{k}(t) & \equiv \boldsymbol{e}^{k}(\boldsymbol{\omega}) \cdot \boldsymbol{B}_{\mathrm{ext}}(\boldsymbol{\omega}, t) .
\end{aligned}
$$

Using the relations (132), (136), (146), and (165) the expression (126) of the external Hamiltonian $H_{\text {ext }}(t)$ is recast as

$$
H_{\mathrm{ext}}(t)=-L_{k} \Omega_{\mathrm{ext}}^{k}(t)-\gamma_{\mathrm{e}} s_{k}^{[n]} B_{\mathrm{ext}}^{k}(t) .
$$


The operators $\Omega_{\text {ext }}^{k}(t)$ and $\omega_{\text {ext }}^{k}(t)$ can be decomposed into square block operators according to

$$
\begin{aligned}
& \Omega_{\mathrm{ext}}^{k}(t)=\left.\bigoplus_{\tilde{s}} \Omega_{\mathrm{ext}}^{\tilde{s} k}(t) \equiv \bigoplus_{\tilde{s}} \mathcal{P}_{B}^{\tilde{s}} \Omega_{\mathrm{ext}}^{k}(t) \mathcal{P}_{B}^{\tilde{s}}\right|_{\mathcal{H}_{B}^{\tilde{s}} \rightarrow \mathcal{H}_{B}^{\tilde{s}}}, \\
& B_{\mathrm{ext}}^{k}(t)=\left.\bigoplus_{\tilde{s}} B_{\mathrm{ext}}^{\tilde{s} k}(t) \equiv \bigoplus_{\tilde{s}} \mathcal{P}_{B}^{\tilde{s}} B_{\mathrm{ext}}^{\tilde{s} k}(t) \mathcal{P}_{B}^{\tilde{s}}\right|_{\mathcal{H}_{B}^{\tilde{s}} \rightarrow \mathcal{H}_{B}^{\tilde{s}}}
\end{aligned}
$$

The substitution of the decompositions (134), (161), and (167) into the expressions (166) yields

$$
H_{\mathrm{ext}}(t)=-\bigoplus_{s} L_{k}^{s} \Omega_{\mathrm{ext}}^{s k}(t)-\gamma_{e} \bigoplus_{\substack{s, s^{\prime} \\ s^{\prime}=s}} s_{k}^{s^{\prime}[n] s} B_{\mathrm{ext}}^{s k}(t) .
$$

\section{Matrix elements of the spin-orbit Hamiltonian}

According to the relation (150), the matrix elements of the spin-orbit Hamiltonian $H_{\text {SO }}$ can be expressed as the sum of the matrix elements of the spin Hamiltonians $H_{\mathrm{SO}}^{[n]}$ and $H_{\mathrm{SO}}^{[n-1,1]}$, i.e.,

$$
\begin{aligned}
& \left\langle a^{\prime}, b^{\prime}, s^{\prime}\left|H_{\mathrm{SO}}\right| a, b, s\right\rangle \\
& \quad=\left\langle a^{\prime}, b^{\prime}, s^{\prime}\left|H_{\mathrm{SO}}^{[n]}\right| a, b, s\right\rangle+\left\langle a^{\prime}, b^{\prime}, s^{\prime}\left|H_{\mathrm{SO}}^{[n-1,1]}\right| a, b, s\right\rangle .
\end{aligned}
$$

Expression (54) for the vectors $|a, b, s\rangle$ and the expression (163) for the operator $H_{\mathrm{SO}}^{[n]}$ imply that the first term on the right-hand side of relation (169) is explicitly expressed as

$$
\begin{aligned}
& \left\langle a^{\prime}, b^{\prime}, s^{\prime}\left|H_{\mathrm{SO}}^{[n]}\right| a, b, s\right\rangle \\
& =-\gamma_{e} \sum_{\substack{i, j=1 \\
i^{\prime}, j^{\prime}=1}}^{d_{s}} c_{i^{\prime} j^{\prime} 1}^{s^{\prime} \tilde{s}^{\prime}\left[1^{n}\right] *} c_{i j 1}^{s \tilde{s}\left[1^{n}\right]}\left\langle a^{\prime}, s^{\prime}, i^{\prime}\left|s_{k}^{s^{\prime}[n] s}\right| a, s, i\right\rangle \\
& \quad \times\left\langle b^{\prime}, \tilde{s}^{\prime}, j^{\prime}\left|\tilde{B}^{\tilde{s}^{\prime}[n] \tilde{s} k}\right| b, \tilde{s}, j\right\rangle .
\end{aligned}
$$

The Wigner-Eckart theorem [37,38], applied to the irreducible representations of the permutation group $S_{n}$, implies that the matrix elements on the right-hand side of relation (170) reduce to

$$
\begin{aligned}
\left\langle a^{\prime}, s^{\prime}, i^{\prime}\left|s_{k}^{s^{\prime}[n] s}\right| a, s, i\right\rangle & =c_{1 i i^{\prime}}^{[n] s s^{\prime}}\left\langle a^{\prime}, s^{\prime}\left|s_{k}^{s^{\prime}[n] s}\right| a, s\right\rangle, \\
\left\langle b^{\prime}, \tilde{s}^{\prime}, j^{\prime}\left|\tilde{B}^{\tilde{s}^{\prime}[n] \tilde{s} k}\right| b, \tilde{s}, j\right\rangle & =c_{1 j j^{\prime}}^{[n] \mid \tilde{s}^{\prime}}\left\langle b^{\prime}, \tilde{s}^{\prime}\left|\tilde{B}^{\tilde{s}^{\prime}[n] \tilde{s} k}\right| b, \tilde{s}\right\rangle,
\end{aligned}
$$

where we used the notation (65). According to the decomposition (161), the spin quantum numbers are equal, i.e., $s^{\prime}=s$, in the relations (171), which implies that the Clebsch-Gordon coefficients $c_{1 i i^{\prime}}^{[n] s s^{\prime}}$ and $c_{1 j j^{\prime}}^{[n]] \tilde{s^{\prime}}}$ are given by

$$
\begin{gathered}
c_{1 i i^{\prime}}^{[n] s s^{\prime}}=\delta^{s^{\prime} s} c_{i^{\prime} i 1}^{s s[n]}=\delta^{s^{\prime} s} \delta_{i^{\prime} i}, \\
c_{1 j j^{\prime}}^{[n] \tilde{s} \tilde{s}^{\prime}}=\delta^{\tilde{s}^{\prime} \tilde{s}} c_{j^{\prime} j 1}^{\tilde{s} \tilde{s}[n]}=\delta^{\tilde{s}^{\prime} \tilde{s}} \delta_{j^{\prime} j},
\end{gathered}
$$

according to the expression (69) since $d_{[n]}=1$. Taking into account the orthonormality conditions (56) for the ClebschGordon coefficients and the reduced matrix elements (171) and the Clebsch-Gordan coefficients (172), the matrix elements (170) reduce to

$$
\begin{aligned}
& \left\langle a^{\prime}, b^{\prime}, s^{\prime}\left|H_{\mathrm{SO}}^{[n]}\right| a, b, s\right\rangle \\
& \quad=-\gamma_{e} \delta^{s^{\prime} s}\left\langle a^{\prime}, s^{\prime}\left|s_{k}^{s^{\prime}[n] s}\right| a, s\right\rangle\left\langle b^{\prime}, \tilde{s}^{\prime}\left|\tilde{B}^{\tilde{s}^{\prime}[n] \tilde{s} k}\right| b, \tilde{s}\right\rangle .
\end{aligned}
$$

Expression (54) for the vectors $|a, b, s\rangle$ and the expression (163) for the operator $H_{\mathrm{SO}}^{[n-1,1]}$ imply that the second term on the right-hand side of relation (169) is explicitly expressed as

$$
\begin{aligned}
& \left\langle a^{\prime}, b^{\prime}, s^{\prime}\left|H_{\mathrm{SO}}^{[n-1,1]}\right| a, b, s\right\rangle \\
& =-\sum_{\substack{i, j=1 \\
i^{\prime}, j^{\prime}=1}}^{d_{s}} c_{i^{\prime} j^{\prime} 1}^{s^{\prime} \tilde{s}^{\prime}\left[1^{n}\right] *} c_{i j 1}^{s \tilde{s}\left[1^{n}\right]} \gamma_{e} \sum_{\ell=1}^{n-1}\left\langle a^{\prime}, b^{\prime}, i^{\prime}\left|s_{k \ell}^{s^{\prime}[n-1,1] s}\right| a, s, i\right\rangle \\
& \quad \times\left\langle b^{\prime}, \tilde{s}^{\prime}, j^{\prime}\left|\tilde{B}_{\ell}^{\tilde{s}^{\prime}[n-1,1] \tilde{s} k}\right| b, \tilde{s}, j\right\rangle .
\end{aligned}
$$

The Wigner-Eckart theorem [37,38], applied to the irreducible representations of the permutation group $S_{n}$, implies that the matrix elements on the right-hand side of relation (174) reduce to

$$
\begin{aligned}
& \left\langle a^{\prime}, s^{\prime}, i^{\prime}\left|s_{k \ell}^{s^{\prime}[n-1,1] s}\right| a, s, i\right\rangle=c_{\ell i i^{\prime}}^{[n-1,1] s s^{\prime}}\left\langle a^{\prime}, s^{\prime}\left|s_{k}^{s^{\prime}[n-1,1] s}\right| a, s\right\rangle, \\
& \left\langle b^{\prime}, \tilde{s}^{\prime}, j^{\prime}\left|\tilde{B}_{\ell}^{\tilde{s}^{\prime}[n] \tilde{s} k}\right| b, \tilde{s}, j\right\rangle=c_{\ell j j^{\prime}}^{[n-1,1] \tilde{s} \tilde{s}^{\prime}}\left\langle b^{\prime}, \tilde{s}^{\prime}\left|\tilde{B}^{\tilde{s}^{\prime}[n-1,1] \tilde{s} k}\right| b, \tilde{s}\right\rangle,
\end{aligned}
$$

where we used the notation (65), and $c_{\ell i i^{\prime}}^{[n-1,1] s s^{\prime}}$ and $c_{\ell j j^{\prime}}^{[n-1,1] \tilde{s} \tilde{s}^{\prime}}$ are the corresponding Clebsch-Gordon coefficients. Taking into account the orthonormality conditions (56) for the Clebsch-Gordon coefficients and the reduced matrix elements (175), the matrix elements (174) reduce to

$$
\begin{aligned}
& \left\langle a^{\prime}, b^{\prime}, s^{\prime}\left|H_{\mathrm{SO}}^{[n-1,1]}\right| a, b, s\right\rangle \\
& =-\gamma_{e} g^{s^{\prime} s}\left\langle a^{\prime}, s^{\prime}\left|s_{k}^{s^{\prime}[n-1,1] s}\right| a, s\right\rangle\left\langle b^{\prime}, \tilde{s}^{\prime}\left|\tilde{B}^{\tilde{s}^{\prime}[n-1,1] \tilde{s} k}\right| b, \tilde{s}\right\rangle,
\end{aligned}
$$

where the coefficients $g^{s^{\prime} s}$ are expressed as a linear combination of products of the Clebsch-Gordon coefficients, i.e.,

$$
\begin{aligned}
g^{s^{\prime} s}= & \sum_{i^{\prime}, j^{\prime}=1}^{d_{s^{\prime}}} \sum_{i, j=1}^{d_{s}} c_{i^{\prime} j^{\prime} 1}^{\left.s^{\prime}\right]^{\prime}\left[1^{n}\right] *} c_{i j 1}^{s \tilde{s}\left[1^{n}\right]} \\
& \times \sum_{\ell=1}^{n-1} c_{\ell i i^{\prime}}^{[n-1,1] s s^{\prime}} c_{\ell j j^{\prime}}^{[n-1,1] \tilde{s} \tilde{s}^{\prime}},
\end{aligned}
$$

where $s^{\prime} \neq s$. The operator $H_{\mathrm{SO}}^{[n-1,1]}$ can change the total electronic spin $s$ of the system $A$. The relation (177) implies that the operator $H_{\mathrm{SO}}^{[n-1,1]}$ is expressed as

$$
H_{\mathrm{SO}}^{[n-1,1]}=-\gamma_{e} \bigoplus_{s, s^{\prime}} g^{s^{\prime} s} s_{k}^{s^{\prime}[n-1,1] s} \otimes \tilde{B}^{\tilde{s}^{\prime}[n-1,1] \tilde{s} k} .
$$

Relations (150), (163), and (179) imply that the spin-orbit Hamiltonian $H_{\text {SO }}$ can be expressed as a direct sum over the types $\mu \in\{[n],[n-1,1]\}$ of the irreducible representations $D^{(\mu)}$ of the permutation group $S_{n}$ as

$$
H_{\mathrm{SO}}=-\gamma_{e} \bigoplus_{\mu \in\{[n],[n-1,1]\}} \bigoplus_{s, s^{\prime}} g^{s^{\prime} \mu s} s_{k}^{s^{\prime} \mu s} \otimes \tilde{B}^{\tilde{s}^{\prime} \mu \tilde{s} k},
$$

where the coefficients $g^{s^{\prime}[n] s}=\delta^{s^{\prime} s}$ and $g^{s^{\prime}[n-1,1] s}=g^{s^{\prime} s}$. 


\section{E. Description of the system $A$}

According to the decomposition (20) and to the identities (26), (28), and (31), the Hilbert space $\mathcal{H}_{A}^{s}$ describing the properties of the system $A$ with a total electronic spin $s$ is expressed as

$$
\mathcal{H}_{A}^{s}=\bigoplus_{a=1}^{2 s+1} \mathcal{H}_{A a}^{s},
$$

where the index $a$ accounts for the multiplicity $2 s+1$ of the irreducible representation $D^{(s)}$ in the Hilbert space $\mathcal{H}_{A}^{s}$. Henceforth, we shall use the magnetic spin quantum number $m$ as label for this multiplicity. According to the relations (13) and (14) with the replacement $\lambda \rightarrow s$, the Hilbert space (181) is recast as

$$
\mathcal{H}_{A}^{s}=\bigoplus_{m=-s}^{s} \mathcal{H}_{\text {def }} \otimes \mathcal{H}_{\text {ori }} \otimes \mathcal{H}_{m}^{s},
$$

where the Hilbert space $\mathcal{H}_{m}^{s}=\mathbb{C}^{d_{s}}$. The vector $|a, s, \tilde{i}\rangle \in \mathcal{H}_{A}^{s}$ is an eigenvector of the Hamiltonian $H_{A}$ of the system $A$, where $a$ labels the physical properties of the system. These properties are given by the set of occupation numbers $\left\{N_{\bullet}\right\}$ of the $3 N-6$ molecular vibration modes, the integers $L, \tilde{M}$, and $\tilde{N}$ describing the molecular orientation and the total electronic spin magnetic quantum number $\tilde{m}$, i.e., $a=\left\{\left\{N_{\bullet}\right\}, L, \tilde{M}, \tilde{N}, \tilde{m}\right\}$, where $-L \leqslant \tilde{M} \leqslant L,-L \leqslant \tilde{N} \leqslant L$, and $-s \leqslant \tilde{m} \leqslant s$. According to the decomposition (182), the eigenvector of the Hamiltonian $H_{A}$, i.e.,

$$
|a, s, \tilde{i}\rangle \equiv\left|\left\{N_{\bullet}\right\}, L, \tilde{M}, \tilde{N}, s, \tilde{m}, \tilde{i}\right\rangle \in \mathcal{H}_{A}^{s},
$$

is written explicitly in terms of the vector $\left|\left\{N_{\bullet}\right\}\right\rangle \in \mathcal{H}_{\text {def }}$, the vector $|L, \tilde{M}, \tilde{N}\rangle \in \mathcal{H}_{\text {ori }}$, and the vector $|s, \tilde{m}, \tilde{i}\rangle \in \mathcal{H}_{m}^{s}$ as

$$
\left|\left\{N_{\bullet}\right\}, L, \tilde{M}, \tilde{N}, s, \tilde{m}, \tilde{i}\right\rangle=\left|\left\{N_{\bullet}\right\}\right\rangle \otimes|L, \tilde{M}, \tilde{N}\rangle \otimes|s, \tilde{m}, \tilde{i}\rangle .
$$

\section{F. Description of the molecular vibrations}

The vector $\left|\left\{N_{\bullet}\right\}\right\rangle \in \mathcal{H}_{\text {def }}$ can be decomposed into a tensor product of vectors associated with the vibrational excitation modes $\alpha=1, \ldots, 3 N-6$, i.e.,

$$
\left|\left\{N_{\bullet}\right\}\right\rangle=\frac{1}{\sqrt{3 N-6}} \bigotimes_{\alpha=1}^{3 N-6}\left|N_{\alpha}\right\rangle,
$$

that satisfy the orthonormality conditions, i.e.,

$$
\begin{aligned}
\left\langle\left\{N_{\bullet}^{\prime}\right\} \mid\left\{N_{\bullet}\right\}\right\rangle & =\prod_{\alpha=1}^{3 N-6} \delta_{N_{\alpha}^{\prime} N_{\alpha}}, \\
\left\langle N_{\alpha}^{\prime} \mid N_{\alpha}\right\rangle & =\delta_{N_{\alpha}^{\prime} N_{\alpha}} \quad \forall \alpha .
\end{aligned}
$$

The set of vectors $\left\{\left|\left\{N_{\bullet}\right\}\right\rangle\right\}$ is an orthonormal basis of the Hilbert space $\mathcal{H}_{\text {def }}=L^{2}\left(\mathbb{R}^{3 N-6}, d x^{3 N-6}\right)$ and the set of vectors $\left\{\left|N_{\alpha}\right\rangle\right\}$ is an orthonormal basis of the Hilbert space $L^{2}(\mathbb{R}, d x)$. The vectors $\left|N_{\alpha}\right\rangle \equiv \psi_{N_{\alpha}}$ are functions represented by

$$
x \mapsto \psi_{N_{\alpha}}(x) .
$$

According to the relations (186) and (187), the coefficients $\psi_{N_{\alpha}}(x)$ satisfy the orthogonality condition,

$$
\int_{\mathbb{R}} \psi_{N_{\alpha}^{\prime}}(x)^{*} \psi_{N_{\alpha}}(x) d x=\delta_{N_{\alpha}^{\prime} N_{\alpha}} .
$$

The annihilation and creation operators $a_{\alpha}$ and $a_{\alpha}^{\dagger}$ of a molecular vibrational excitation in the mode $\alpha$ are defined as, i.e.,

$$
\begin{aligned}
& a_{\alpha}=\frac{1}{\sqrt{2 \hbar \omega_{\alpha}}}\left(P_{\alpha}-i \omega_{\alpha} Q_{\alpha}\right), \\
& a_{\alpha}^{\dagger}=\frac{1}{\sqrt{2 \hbar \omega_{\alpha}}}\left(P_{\alpha}+i \omega_{\alpha} Q_{\alpha}\right),
\end{aligned}
$$

where $\omega_{\alpha}$ is the angular frequency of the vibration mode $\alpha$, which implies that

$$
\begin{aligned}
P_{\alpha} & =\sqrt{\frac{\hbar \omega_{\alpha}}{2}}\left(a_{\alpha}+a_{\alpha}^{\dagger}\right), \\
Q_{\alpha} & =i \sqrt{\frac{\hbar}{2 \omega_{\alpha}}}\left(a_{\alpha}-a_{\alpha}^{\dagger}\right) .
\end{aligned}
$$

The canonical commutation relations read

$$
\left[P_{\alpha}, Q^{\beta}\right]=-i \hbar \delta_{\alpha}^{\beta} \mathbb{1} \text {. }
$$

The definitions (191) and (192) and the canonical commutation relations (193) imply that

$$
\left[a_{\alpha}, a_{\alpha^{\prime}}^{\dagger}\right]=\delta_{\alpha \alpha^{\prime}} \mathbb{1} .
$$

The self-adjoint and positive definite operator $\mathcal{N}_{\alpha}$ associated with the molecular vibrational occupation number in the mode $\omega_{\alpha}$ is defined as

$$
\mathcal{N}_{\alpha}=a_{\alpha}^{\dagger} a_{\alpha}
$$

The basis vectors $\left|\left\{N_{\bullet}\right\}\right\rangle$ are eigenvectors of the operator $\mathcal{N}_{\alpha}=$ $a_{\alpha}^{\dagger} a_{\alpha}$. The corresponding eigenvalue equation is given by

$$
\mathcal{N}_{\alpha}\left|\left\{N_{\bullet}\right\}\right\rangle=N_{\alpha}\left|\left\{N_{\bullet}\right\}\right\rangle,
$$

where $N_{\alpha}$ is the occupation number of the mode $\alpha$. The action of the annihilation and creation operators on the eigenvector $\left|\left\{N_{\bullet}\right\}\right\rangle$ is given by

$$
\begin{aligned}
& a_{\alpha}\left|\left\{N_{\bullet}\right\}\right\rangle=\sqrt{N_{\alpha}}\left|\left\{N_{\bullet}-\delta_{\bullet}^{\alpha}\right\}\right\rangle, \\
& a_{\alpha}^{\dagger}\left|\left\{N_{\bullet}\right\}\right\rangle=\sqrt{N_{\alpha}+1}\left|\left\{N_{\bullet}+\delta_{\bullet}^{\alpha}\right\}\right\rangle .
\end{aligned}
$$

The definition (192) of the molecular deformation operator $Q_{\alpha}$ in the vibrational mode $\alpha$ and the action (197) of the creation and annihilation operators $a_{\alpha}^{\dagger}$ and $a_{\alpha}$ on the vector $\left|\left\{N_{\bullet}\right\}\right\rangle$ imply that the matrix elements of the molecular deformation operator $Q_{\alpha}$ have to satisfy the condition

$$
\left\langle\left\{N_{\bullet} \pm \delta_{\bullet}^{\alpha}\right\}\left|Q_{\alpha}\right|\left\{N_{\bullet}\right\}\right\rangle=\mp i c_{ \pm}^{\omega_{\alpha} N_{\alpha}},
$$

where the coefficients $c_{ \pm}^{\omega_{\alpha} N_{\alpha}}$ defined as

$$
c_{ \pm}^{\omega_{\alpha} N_{\alpha}}=\sqrt{\frac{N_{\alpha} \pm \frac{1}{2}(1 \pm 1)}{\hbar \omega_{\alpha}}}
$$

satisfy the condition

$$
c_{+}^{\omega_{\alpha} N_{\alpha}}=c_{-}^{\omega_{\alpha} N_{\alpha}+1} .
$$


The matrix elements (198) impose the molecular vibration selection rules on the occupation numbers $N_{\alpha}, N_{\alpha}^{\prime}, N_{\beta}$, and $N_{\beta}^{\prime}$ of the vibration modes $\alpha$ and $\beta \neq \alpha$,

$$
\left|N_{\alpha}^{\prime}-N_{\alpha}\right| \leqslant 1 \quad \text { and } \quad\left|N_{\beta}^{\prime}-N_{\beta}\right|=0 \quad \forall \beta \neq \alpha .
$$

\section{G. Description of the molecular rotations}

The set of vectors $\{|L, M, N\rangle\}$ is an orthonormal basis of the Hilbert space $\mathcal{H}_{\text {ori }}=L^{2}(\operatorname{SO}(3), \sin \theta d \psi d \theta d \varphi)$, i.e.,

$$
\left\langle L^{\prime}, M^{\prime}, N^{\prime} \mid L, M, N\right\rangle=\delta^{L^{\prime} L} \delta_{M^{\prime} M} \delta_{N^{\prime} N} .
$$

The vectors $|L, M, N\rangle$ are defined in terms of the matrix elements $d_{M N}^{L}$ of the irreducible representation $D^{(L)}$ of the rotation group $\mathrm{SO}(3)$ as

$$
|L, M, N\rangle \equiv \sqrt{\frac{2 L+1}{8 \pi^{2}}} d_{M N}^{L},
$$

where the function $d_{M N}^{L}$ defined as

$$
(\psi, \theta, \varphi) \mapsto d_{M N}^{L}(\psi, \theta, \varphi)
$$

yields the Wigner $d$-matrix elements $d_{M N}^{L}(\psi, \theta, \varphi)$ [39]. According to the relations (202), (203), and (204), the coefficients $d_{M N}^{L}(\psi, \theta, \varphi)$ satisfy the orthogonality condition,

$$
\begin{aligned}
& \int_{\mathrm{SO}(3)} d_{M^{\prime} N^{\prime}}^{L^{\prime}}(\psi, \theta, \varphi)^{*} d_{M N}^{L}(\psi, \theta, \varphi) \sin \theta d \psi d \theta d \varphi \\
& =\frac{8 \pi^{2}}{2 L+1} \delta^{L^{\prime} L} \delta_{M^{\prime} M} \delta_{N^{\prime} N}
\end{aligned}
$$

The rotational part of the free Hamiltonian (115) of the system is expressed as

$$
H_{A}^{\mathrm{rot}}=\sum_{k=1}^{3}\left(\frac{L_{k}^{2}}{2 I_{0 k}}-\left\langle\Omega^{k}\right\rangle L_{k}\right)
$$

where $I_{0 k}=\boldsymbol{e}_{k}(\boldsymbol{\omega}) \cdot I_{0} \cdot \boldsymbol{e}_{k}(\boldsymbol{\omega})$ is a moment of inertia of the molecule. The eigenvectors $|L, \tilde{M}, \tilde{N}\rangle$ of the Hamiltonian $H_{A}^{\text {rot }}$ are related to the vectors $|L, M, N\rangle$ by a unitary transformation in a Hilbert space of dimension $(2 L+1)^{2}$, i.e.,

$$
|L, \tilde{M}, \tilde{N}\rangle=\sum_{M=-L}^{L} \sum_{N=-L}^{L} c_{M N}^{\tilde{M} \tilde{N}}|L, M, N\rangle,
$$

where the coefficients $c_{M N}^{\tilde{M} \tilde{N}}$ satisfy the orthogonality condition,

$$
\sum_{M=-L}^{L} \sum_{N=-L}^{L} c_{M N}^{\tilde{M} \tilde{N} *} c_{M N}^{\tilde{M} \tilde{N}}=1
$$

The irreducible representation $D^{(L)}(\psi, \theta, \varphi)$ of the rotation group $\mathrm{SO}(3)$ is parametrized in terms of the Euler angles $(\psi, \theta, \varphi)$ as

$$
D^{(L)}(\psi, \theta, \varphi)=e^{-\frac{i}{\hbar} \psi L_{3}} e^{-\frac{i}{\hbar} \theta L_{2}} e^{-\frac{i}{\hbar} \varphi L_{3}},
$$

where the molecular orbital angular momentum operators $L_{1}$, $L_{2}$, and $L_{3}$ given by [40]

$$
\begin{aligned}
& L_{1}=i \hbar\left(\frac{\cos \varphi}{\sin \theta} \frac{\partial}{\partial \psi}-\sin \varphi \frac{\partial}{\partial \theta}-\frac{\cos \varphi}{\tan \theta} \frac{\partial}{\partial \varphi}\right), \\
& L_{2}=i \hbar\left(\frac{\sin \varphi}{\sin \theta} \frac{\partial}{\partial \psi}+\cos \varphi \frac{\partial}{\partial \theta}-\frac{\sin \varphi}{\tan \theta} \frac{\partial}{\partial \varphi}\right), \\
& L_{3}=i \hbar \frac{\partial}{\partial \varphi}
\end{aligned}
$$

satisfy the anomalous commutation relations (i.e., with a negative sign on the right-hand side),

$$
\left[L_{1}, L_{2}\right]=-i \hbar L_{3} \text {. }
$$

The complex coefficients $d_{M N}^{L}(\psi, \theta, \varphi)$ have the property

$$
d_{M N}^{L}(\psi, \theta, \varphi)=d_{N M}^{L}(\varphi, \theta, \psi) .
$$

Taking into account the property, the irreducible representation $D^{(L)}(\varphi, \theta, \psi)$ of the rotation group $\mathrm{SO}(3)$ is parametrized in terms of the Euler angles $(\varphi, \theta, \psi)$ as

$$
D^{(L)}(\psi, \theta, \varphi)=e^{-\frac{i}{\hbar} \varphi \tilde{L}_{3}} e^{-\frac{i}{\hbar} \theta \tilde{L}_{2}} e^{-\frac{i}{\hbar} \psi \tilde{L}_{3}},
$$

where the molecular orbital angular momentum operators $\tilde{L}_{1}, \tilde{L}_{2}$, and $\tilde{L}_{3}$ are obtained from the molecular orbital angular momentum operators $L_{1}, L_{2}$, and $L_{3}$, respectively, by interchanging the precession and eigenrotation angles, i.e., $\psi \leftrightarrow \varphi$,

$$
\begin{aligned}
& \tilde{L}_{1}=i \hbar\left(-\frac{\cos \psi}{\tan \theta} \frac{\partial}{\partial \psi}-\sin \psi \frac{\partial}{\partial \theta}+\frac{\cos \psi}{\sin \theta} \frac{\partial}{\partial \varphi}\right) \\
& \tilde{L}_{2}=i \hbar\left(-\frac{\sin \psi}{\tan \theta} \frac{\partial}{\partial \psi}+\cos \psi \frac{\partial}{\partial \theta}+\frac{\sin \psi}{\sin \theta} \frac{\partial}{\partial \varphi}\right), \\
& \tilde{L}_{3}=i \hbar \frac{\partial}{\partial \psi}
\end{aligned}
$$

These operators satisfy the anomalous commutation relations (i.e., with a negative sign on the right-hand side),

$$
\left[\tilde{L}_{1}, \tilde{L}_{2}\right]=-i \hbar \tilde{L}_{3} \text {. }
$$

The molecular orbital angular momentum operators $L_{1}, L_{2}$, $L_{3}$ and $\tilde{L}_{1}, \tilde{L}_{2}, \tilde{L}_{3}$ commute; i.e.,

$$
\left[L_{i}, \tilde{L}_{k}\right]=0 \quad \forall i, k=1, \ldots, 3 .
$$

The Casimir operator $L^{2}=L_{1}^{2}+L_{2}^{2}+L_{3}^{2}$ of the Lie algebra $\mathfrak{s o}(3)$ is expressed in terms of the molecular orbital angular momentum operators as [40]

$$
L^{2}=L_{1}^{2}+L_{2}^{2}+L_{3}^{2}=\tilde{L}_{1}^{2}+\tilde{L}_{2}^{2}+\tilde{L}_{3}^{2} .
$$

The expressions (210), (214), and (217) imply that the Casimir operator is explicitly expressed in terms of the Euler angles as

$$
\begin{aligned}
L^{2}= & -\hbar^{2}\left[\frac{1}{\sin \theta} \frac{\partial}{\partial \theta}\left(\sin \theta \frac{\partial}{\partial \theta}\right)\right. \\
& \left.+\frac{1}{\sin ^{2} \theta}\left(\frac{\partial^{2}}{\partial \psi^{2}}-2 \cos \theta \frac{\partial}{\partial \psi \partial \varphi}+\frac{\partial^{2}}{\partial \varphi^{2}}\right)\right]
\end{aligned}
$$

and commutes with the molecular orbital angular momentum operators, i.e.,

$$
\left[L^{2}, L_{i}\right]=\left[L^{2}, \tilde{L}_{i}\right]=0 \quad \forall i=1,2,3 .
$$


The normalized vectors $|L, M, N\rangle$ are eigenvectors of the operators $L^{2}, L_{3}$, and $\tilde{L}_{3}$ [40], i.e.,

$$
\begin{aligned}
& L^{2}|L, M, N\rangle=\hbar^{2} L(L+1)|L, M, N\rangle, \\
& L_{3}|L, M, N\rangle=\hbar M|L, M, N\rangle, \\
& \tilde{L}_{3}|L, M, N\rangle=\hbar N|L, M, N\rangle .
\end{aligned}
$$

The operators $L_{ \pm}$and $\tilde{L}_{ \pm}$are defined as

$$
L_{ \pm}=L_{1} \mp i L_{2} \quad \text { and } \quad \tilde{L}_{ \pm}=\tilde{L}_{1} \mp i \tilde{L}_{2}
$$

Thus, $L_{\mp}=L_{ \pm}^{\dagger}$ and $\tilde{L}_{\mp}=\tilde{L}_{ \pm}^{\dagger}$, which implies that

$$
\begin{aligned}
L_{1} & =\frac{1}{2}\left(L_{+}+L_{-}\right) \quad \text { and } \quad L_{2}=-\frac{i}{2}\left(L_{+}-L_{-}\right), \\
\tilde{L}_{1} & =\frac{1}{2}\left(\tilde{L}_{+}+\tilde{L}_{-}\right) \quad \text { and } \quad \tilde{L}_{2}=-\frac{i}{2}\left(\tilde{L}_{+}-\tilde{L}_{-}\right) .
\end{aligned}
$$

The action of the operators (223) on the vectors $|L, M, N\rangle$ is expressed as

$$
\begin{aligned}
& L_{ \pm}|L, M, N\rangle=\hbar c_{ \pm}^{L M}|L, M \pm 1, N\rangle \\
& \tilde{L}_{ \pm}|L, M, N\rangle=\hbar c_{ \pm}^{L N}|L, M, N \pm 1\rangle
\end{aligned}
$$

where the real coefficients $c_{ \pm}^{L M}$ and $c_{ \pm}^{L N}$ given by

$$
\begin{aligned}
c_{ \pm}^{L M} & =\sqrt{L(L+1)-M(M \pm 1)} \\
& =\sqrt{(L \mp M)(L \pm M+1)} \\
c_{ \pm}^{L N} & =\sqrt{L(L+1)-N(N \pm 1)} \\
& =\sqrt{(L \mp N)(L \pm N+1)},
\end{aligned}
$$

satisfy the conditions

$$
c_{+}^{L M}=c_{-}^{L M+1} \quad \text { and } \quad c_{+}^{L N}=c_{-}^{L N+1} .
$$

The constraints $-L \leqslant M \leqslant L$ and $-L \leqslant N \leqslant L$ give rise to the conditions,

$$
\begin{array}{lll}
L_{-}|0,0, N\rangle=0 & \text { and } & L_{ \pm}|L, \pm L, N\rangle=0, \\
\tilde{L}_{-}|0, M, 0\rangle=0 & \text { and } & \tilde{L}_{ \pm}|L, M, \pm L\rangle=0 .
\end{array}
$$

The action (228) of the molecular orbital angular momentum operators $L_{ \pm}$and $\tilde{L}_{ \pm}$on the vector $|L, M, N\rangle$ imply that the matrix elements of the molecular orbital angular momentum operators $L_{ \pm}$and $\tilde{L}_{ \pm}$are given by

$$
\begin{aligned}
& \left\langle L, M \pm 1, N\left|L_{ \pm}\right| L, M, N\right\rangle=\hbar c_{ \pm}^{L M}, \\
& \left\langle L, M, N \pm 1\left|\tilde{L}_{ \pm}\right| L, M, N\right\rangle=\hbar c_{ \pm}^{L N} .
\end{aligned}
$$

The matrix elements (231) impose the molecular rotation rules on the quantum numbers $L, L^{\prime}, M, M^{\prime}, N$, and $N^{\prime}$, i.e.,

$$
\left|L^{\prime}-L\right|=0, \quad\left|M^{\prime}-M\right| \leqslant 1, \quad\left|N^{\prime}-N\right| \leqslant 1 .
$$

\section{H. Description of the electronic spins}

The set of vectors $\{|s, m, i\rangle\}$ is an orthonormal basis of the Hilbert space $\mathbb{C}^{d_{s}}$, i.e.,

$$
\left\langle s^{\prime}, m^{\prime}, i^{\prime} \mid s, m, i\right\rangle=\delta^{s^{\prime} s} \delta_{m^{\prime} m} \delta_{i^{\prime} i},
$$

according to the relation (50). Taking into account the condition (233), the set of vectors $\{|s, m\rangle \equiv|s, m, 1\rangle\}$ satisfies the orthonormality condition, i.e.,

$$
\left\langle s^{\prime}, m^{\prime} \mid s, m\right\rangle=\delta^{s^{\prime} s} \delta_{m^{\prime} m} .
$$

The spin-orbit part of the free Hamiltonian (115) of the system is expressed as

$$
H_{A}^{\mathrm{SO}}=-\sum_{\nu=1}^{n} \sum_{k=1}^{3}\left\langle B_{\nu}^{k}\right\rangle s_{\nu k} .
$$

The eigenvectors $|s, \tilde{m}, \tilde{i}\rangle$ of the Hamiltonian $H_{A}^{\text {SO }}$ are related to the vectors $|s, m, i\rangle$ by a unitary transformation in a Hilbert space of dimension $(2 s+1) d_{s}$, i.e.,

$$
|s, \tilde{m}, \tilde{i}\rangle=\sum_{m=-s}^{s} \sum_{i=1}^{d_{s}} c_{m i}^{\tilde{m} \tilde{i}}|s, m, i\rangle
$$

where the coefficients $c_{m i}^{\tilde{m} \tilde{i}}$ satisfy the orthogonality condition,

$$
\sum_{m=-s}^{s} \sum_{i=1}^{d_{s}} c_{m i}^{\tilde{m} \tilde{i} *} c_{m i}^{\tilde{m} \tilde{i}}=1 .
$$

The operators $s_{v \pm}$ associated with the electron $v$ are defined as

$$
s_{\nu \pm}=s_{\nu 1} \pm i s_{\nu 2} .
$$

Thus, $s_{v \pm}=s_{v \mp}^{\dagger}$, which implies that

$$
s_{\nu 1}=\frac{1}{2}\left(s_{\nu+}+s_{\nu-}\right), \quad s_{\nu 2}=-\frac{i}{2}\left(s_{\nu+}-s_{\nu-}\right) .
$$

The commutation relations between the components of the spin operator associated with the electron $v$ read [30]

$$
\left[s_{v i}, s_{v j}\right]=i \hbar \varepsilon_{i j k} s_{v}^{k} .
$$

The definitions (239) and the commutation relations (240) imply that the operators $s_{v-}, s_{v+}$, and $s_{v 3}$ associated with the electron $v$ satisfy the commutation relations

$$
\left[s_{v 3}, s_{v^{\prime} \pm}\right]= \pm \delta_{v v^{\prime}} \hbar s_{v^{\prime} \pm}, \quad\left[s_{v+}, s_{v^{\prime}-}\right]=2 \delta_{v v^{\prime}} \hbar s_{v^{\prime} 3} .
$$

The components of the spin operator $s$ associated with all the electrons are written as

$$
s_{k}=\sum_{\nu=1}^{n} s_{\nu k} .
$$

The operators $s_{ \pm}$are defined as

$$
s_{ \pm}=s_{1} \pm i s_{2} .
$$

Thus, $s_{ \pm}=s_{\mp}^{\dagger}$, which implies that

$$
s_{1}=\frac{1}{2}\left(s_{+}+s_{-}\right), \quad s_{2}=-\frac{i}{2}\left(s_{+}-s_{-}\right) .
$$

The commutation relations between the components of the spin operator read

$$
\left[s_{i}, s_{j}\right]=i \hbar \varepsilon_{i j k} s^{k} .
$$

The definitions (244) and the commutation relations (245) imply that the operators $s_{-}, s_{+}$, and $s_{3}$ satisfy the commutation relations

$$
\left[s_{3}, s_{ \pm}\right]= \pm \hbar s_{ \pm}, \quad\left[s_{+}, s_{-}\right]=2 \hbar s_{3} .
$$


The basis vectors $|s, m, i\rangle$ are eigenvectors of the operators $s^{2}$ and $s_{3}$. The corresponding eigenvalue equations are given by

$$
s^{2}|s, m, i\rangle=\hbar^{2} s(s+1)|s, m, i\rangle, \quad s_{3}|s, m, i\rangle=\hbar m|s, m, i\rangle .
$$

The action of the operator $s_{ \pm}$on the eigenvector $|s, m, i\rangle$ is given by

$$
s_{ \pm}|s, m, i\rangle=\hbar c_{ \pm}^{s m}|s, m \pm 1, i\rangle,
$$

where the coefficients $c_{ \pm}^{s m}$ defined as

$$
c_{ \pm}^{s m}=\sqrt{s(s+1)-m(m \pm 1)}=\sqrt{(s \mp m)(s \pm m+1)}
$$

satisfy the condition

$$
c_{+}^{s m}=c_{-}^{s m+1} .
$$

Since the electronic magnetic number $m$ is bounded by $-s \leqslant$ $m \leqslant s$, we also have the conditions

$$
s_{-}|0,0, i\rangle=0 \quad \text { and } \quad s_{ \pm}|s, \pm s, i\rangle=0
$$

\section{Spin selection rules}

The vectors $|s, m, i\rangle \in \mathcal{H}_{m}^{s}$ are invariant under the action of the irreducible representation $D^{(s)} \equiv D^{[n / 2+s, n / 2-s]}$ of the permutation group $S_{n}$. The spin operators $s_{k}^{s^{\prime}[n-1,1] s}$ are invariant under the action of the irreducible representation $D^{[n-1,1]}$ of the permutation group $S_{n}$. The action of the spin operator $s_{k}^{s^{\prime}[n-1,1] s}$ on the vector $|s, m, i\rangle$ yields a vector $\left|s^{\prime}, m^{\prime}, i^{\prime}\right\rangle \in \mathcal{H}_{m^{\prime}}^{s^{\prime}}$ that is invariant under the action of the irreducible representation $D^{\left(s^{\prime}\right)} \equiv D^{\left[n / 2+s^{\prime}, n / 2-s^{\prime}\right]}$ of the permutation group $S_{n}$ :

$$
\left|s^{\prime}, m^{\prime}, i^{\prime}\right\rangle=s_{k}^{s^{\prime}[n-1,1] s}|s, m, i\rangle .
$$

Taking into account relation (252), the tensor product of the irreducible representations $D^{[n-1,1]}$ and $D^{[n / 2+s, n / 2-s]}$ of the permutation group $S_{n}$ is isomorphic to the irreducible representation $D^{\left[n / 2+s^{\prime}, n / 2-s^{\prime}\right]}$, i.e.,

$$
D^{[n-1,1]} \otimes D^{[n / 2+s, n / 2-s]}=\bigoplus_{s^{\prime}} a_{\left[n / 2+s^{\prime}, n / 2-s^{\prime}\right]} D^{\left[n / 2+s^{\prime}, n / 2-s^{\prime}\right]},
$$

where the multiplicity $a_{\left[n / 2+s^{\prime}, n / 2-s^{\prime}\right]}$ of the irreducible representation $D^{\left[n / 2+s^{\prime}, n / 2-s^{\prime}\right]}$ satisfies the orthogonality condition [29],

$$
\begin{aligned}
& a_{\left[n / 2+s^{\prime}, n / 2-s^{\prime}\right]} \\
& \quad=\frac{1}{n !} \sum_{\sigma \in S_{n}} \chi_{\left[n / 2+s^{\prime}, n / 2-s^{\prime}\right]}(\sigma)^{*} \chi_{[n-1,1]}(\sigma) \chi_{[n / 2+s, n / 2-s]}(\sigma) \\
& \quad=\frac{1}{n !} \sum_{\sigma \in S_{n}} \chi_{\left[n / 2+s^{\prime}, n / 2-s^{\prime}\right]}(\sigma)^{*} \chi_{[n / 2+s \pm 1, n / 2-s \mp 1]}(\sigma) \\
& \quad=\delta_{s^{\prime} s \pm 1} .
\end{aligned}
$$

This yields the following selection rule on the spin quantum numbers $s$ and $s^{\prime}[41,42]$,

$$
\left|s^{\prime}-s\right|=1 \quad \text { if } s^{\prime} \neq s .
$$

The Wigner-Eckart theorem [37,38], applied to the irreducible representations of the rotation group $\mathrm{SO}(3)$ relates the matrix elements and the reduced matrix elements of the spin operator $s_{k}^{s^{\prime} \mu s}$ in the following way:

$$
\left\langle s^{\prime}, m^{\prime}\left|s_{k}^{s^{\prime} \mu s}\right| s, m\right\rangle=c_{k m m^{\prime}}^{1 s s^{\prime}}\left\langle s^{\prime}\left\|s^{s^{\prime} \mu s}\right\| s\right\rangle,
$$

where $c_{k m m^{\prime}}^{1 s s^{\prime}}$ are the Clebsch-Gordon coefficients associated with the equivalence between the irreducible representation $D^{\left(s^{\prime}\right)}$ and the tensor product of the irreducible representations $D^{(1)}$ and $D^{(s)}$ of the rotation group $\mathrm{SO}(3)$. These coefficients satisfy the condition

$$
c_{k m m^{\prime}}^{1 s s^{\prime}}=0 \quad \text { if } m^{\prime} \neq m+k,
$$

where $k=-1,0,1$. The condition (257) yields the magnetic spin selection rule

$$
\left|m^{\prime}-m\right| \leqslant 1
$$

which holds for the spin operators $s^{s^{\prime}[n] s}$ and $s^{s^{\prime}[n-1,1] s}$ acting on a vector $|s, m\rangle$.

\section{J. Matrix elements of the spin operators}

According to relation (155),

$$
\sum_{\ell=1}^{n-1} a_{\ell}^{v} s_{k \ell}^{[n-1,1]}=\sum_{\ell=1}^{n-1} a_{\ell}^{v}\left(\sum_{\nu^{\prime}=1}^{n} a_{\ell}^{v^{\prime}} s_{\nu^{\prime} k}\right)
$$

Using property (158), it reduces to

$$
\sum_{\ell=1}^{n-1} a_{\ell}^{v} s_{k \ell}^{[n-1,1]}=\sum_{\nu^{\prime}=1}^{n}\left(\delta^{\nu v^{\prime}}-\frac{1}{n}\right) s_{v^{\prime} k}
$$

Using the first expression (146), the relation (260) becomes

$$
\sum_{\ell=1}^{n-1} a_{\ell}^{v} s_{k \ell}^{[n-1,1]}=\sqrt{n}\left(s_{\nu k}-\frac{1}{n} s_{k}^{[n]}\right) .
$$

Taking into account the decompositions (161) and (162), it yields

$$
\begin{aligned}
& \sum_{\ell=1}^{n-1} a_{\ell}^{v}\left\langle s^{\prime}, m^{\prime}, i^{\prime}\left|s_{k \ell}^{s^{\prime}[n-1,1] s}\right| s, m, i\right\rangle \\
& \quad=\sqrt{n}\left\langle s^{\prime}, m^{\prime}, i^{\prime}\left|s_{v k}\right| s, m, i\right\rangle-\frac{1}{\sqrt{n}}\left\langle s^{\prime}, m^{\prime}, i^{\prime}\left|s_{k}^{s^{\prime}[n] s}\right| s, m, i\right\rangle .
\end{aligned}
$$

Using the reduced matrix elements (171), (172), and (175) and taking the sum with respect to the indices $i$ and $i^{\prime}$, where $i=i^{\prime}$ for the last term on the right-hand side, the relation (262) is 
recast as

$$
\begin{aligned}
\sum_{\ell=1}^{n-1} a_{\ell}^{v} \sum_{i^{\prime}=1}^{d_{s^{\prime}}} \sum_{i=1}^{d_{s}} c_{\ell i i^{\prime}}^{[n-1,1] s s^{\prime}}\left\langle s^{\prime}, m^{\prime}\left|s_{k}^{s^{\prime}[n-1,1] s}\right| s, m\right\rangle \\
=\sqrt{n} \sum_{i^{\prime}=1}^{d_{s^{\prime}}} \sum_{i=1}^{d_{s}}\left\langle s^{\prime}, m^{\prime}, i^{\prime}\left|s_{v k}\right| s, m, i\right\rangle \\
-\frac{d_{s}}{\sqrt{n}} \delta^{s^{\prime} s}\left\langle s^{\prime}, m^{\prime}\left|s_{k}^{s^{\prime}[n] s}\right| s, m\right\rangle .
\end{aligned}
$$

The definition (161) and (162) of the spin operators $s_{k}^{s^{\prime}[n] s}$ and $s_{k}^{s^{\prime}[n-1,1] s}$ and the spin selection rule (255) imply that the first vanishes if $s^{\prime}=s \pm 1$ and the second vanishes if $s^{\prime}=s$. Moreover, the relation (263) holds for every electron $v$. Thus, for the electron $v=1$, relation (263) yields an expression for the spin operator $s_{k}^{s^{\prime}[n] s}$ invariant under the action of the representation of type $[n]$ of the permutation group $S_{n}$, i.e.,

$$
\left\langle s^{\prime}, m^{\prime}\left|s_{k}^{s^{\prime}[n] s}\right| s, m\right\rangle=\frac{n}{d_{s}} \delta^{s^{\prime} s} \sum_{i^{\prime}=1}^{d_{s^{\prime}}} \sum_{i=1}^{d_{s}}\left\langle s^{\prime}, m^{\prime}, i^{\prime}\left|s_{1 k}\right| s, m, i\right\rangle,
$$

and another expression for the spin operator $s_{k}^{s^{\prime}[n-1,1] s}$ invariant under the action of the representation of type $[n-1,1]$ of the permutation group $S_{n}$, i.e.,

$$
\begin{aligned}
& \left\langle s^{\prime}, m^{\prime}\left|s_{k}^{s^{\prime}[n-1,1] s}\right| s, m\right\rangle \\
& =\frac{n}{d_{s}} \delta^{s^{\prime} s \pm 1} \lambda_{n}^{s^{\prime} s} \sum_{i^{\prime}=1}^{d_{s^{\prime}}} \sum_{i=1}^{d_{s}}\left\langle s^{\prime}, m^{\prime}, i^{\prime}\left|s_{1 k}\right| s, m, i\right\rangle,
\end{aligned}
$$

where the numerical factor $\lambda_{n}^{s^{\prime} s}$ is given by

$$
\lambda_{n}^{s^{\prime} s}=\left(\sqrt{n} \sum_{\ell=1}^{n-1} a_{\ell}^{1} \sum_{j=1}^{d_{s}} \sum_{j^{\prime}=1}^{d_{s^{\prime}}} c_{\ell j j^{\prime}}^{[n-1,1] s s^{\prime}}\right)^{-1} .
$$

According to the spin selection rules (255) and (258), there are eight allowed electronic spin transitions $|s, m\rangle \rightarrow\left|s^{\prime}, m^{\prime}\right\rangle$, where $\left|s^{\prime}-s\right| \leqslant 1$ and $\left|m^{\prime}-m\right| \leqslant 1$, which correspond to nearest-neighbor states on the $(s, m)$ plane, as shown in Fig. 2.

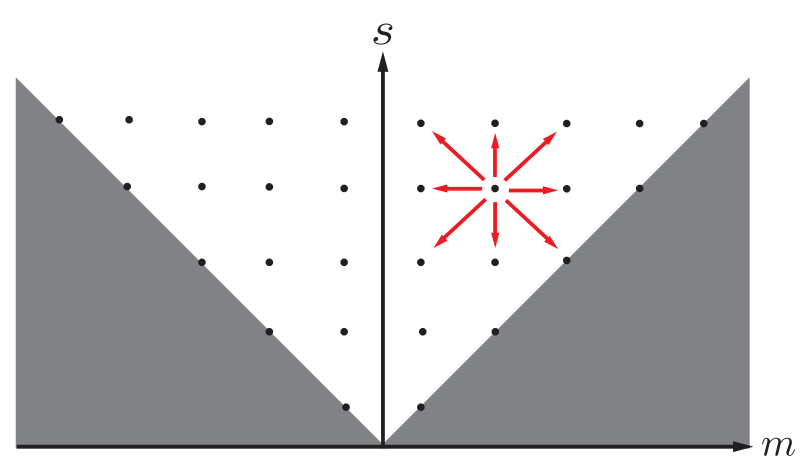

FIG. 2. Electronic spin transitions $|s, m\rangle \rightarrow\left|s^{\prime}, m^{\prime}\right\rangle$, where $\left|s^{\prime}-s\right|$ $\leqslant 1$ and $\left|m^{\prime}-m\right| \leqslant 1$ according to the spin selection rules for an odd number $n$ of electrons.
The amplitudes of the electronic spin transitions $|s, m\rangle \rightarrow$ $|s, m \pm 1\rangle$ are given by the matrix elements of the spin operators $s_{ \pm}^{s[n] s} \equiv s_{ \pm}$. Taking into account the expression (250) for the coefficient $c_{+}^{s m}$, the substitution of the reduced matrix element (B137) established in Appendix B into relation (264) yields

$$
\left\langle s, m \pm 1\left|s_{ \pm}^{s[n] s}\right| s, m\right\rangle=\hbar c_{ \pm}^{s m}
$$

The amplitudes of the electronic spin transitions $|s, m\rangle \rightarrow$ $|s-1, m\rangle$ and $|s, m\rangle \rightarrow|s-1, m \pm 1\rangle$ are given by the matrix elements of the spin operators $s_{3}^{s-1[n-1,1] s}$ and $s_{ \pm}^{s-1[n-1,1] s}$. Taking into account the expressions (30), (B49), and (B89), the substitution of the reduced matrix elements (B136) and (B137) established in Appendix B into relation (265) yields

$$
\begin{aligned}
& \left\langle s-1, m\left|s_{3}^{s-1[n-1,1] s}\right| s, m\right\rangle=\hbar c_{s-1 m}^{s m} \\
& \left\langle s-1, m \pm 1\left|s_{ \pm}^{s-1[n-1,1] s}\right| s, m\right\rangle= \pm \hbar c_{s-1 m \pm 1}^{s m}
\end{aligned}
$$

where the coefficients are given by

$$
\begin{aligned}
& c_{s-1 m}^{s m}=\lambda_{n}^{s-1 s} \frac{\frac{n}{2}+s+1}{2 s+1} \sqrt{(s-m)(s+m)}, \\
& c_{s-1 m \pm 1}^{s m}= \pm \lambda_{n}^{s-1 s} \frac{\frac{n}{2}+s+1}{2 s+1} \sqrt{(s+1 \mp m)(s \mp m)} .
\end{aligned}
$$

The amplitudes of the electronic spin transitions $|s, m\rangle \rightarrow$ $|s+1, m\rangle$ and $|s, m\rangle \rightarrow|s+1, m \pm 1\rangle$ are given by the matrix elements of the spin operators $s_{3}^{s+1[n-1,1] s}$ and $s_{ \pm}^{s+1[n-1,1] s}$. Taking into account the expressions (30), (B48), and (B89) the substitution of the reduced matrix elements (B136) and (B137) established in Appendix B into relation (265) yields

$$
\begin{aligned}
& \left\langle s+1, m\left|s_{3}^{s-1[n-1,1] s}\right| s, m\right\rangle=\hbar c_{s+1 m}^{s m} \\
& \left\langle s+1, m \pm 1\left|s_{ \pm}^{s-1[n-1,1] s}\right| s, m\right\rangle=\mp \hbar c_{s+1 m \pm 1}^{s m}
\end{aligned}
$$

where the coefficients are given by

$$
\begin{aligned}
& c_{s+1 m}^{s m}=\lambda_{n}^{s+1 s} \frac{\frac{n}{2}-s}{2 s+1} \sqrt{(s+1-m)(s+1+m)}, \\
& c_{s+1 m \pm 1}^{s m}=\mp \lambda_{n}^{s+1 s} \frac{\frac{n}{2}-s}{2 s+1} \sqrt{(s+1 \pm m)(s+2 \pm m)} .
\end{aligned}
$$

\section{TRANSITION RATES AND DIAGRAMS}

\section{A. Diagram elements}

The molecular vibrational transitions $\left|\left\{N_{\bullet}\right\}\right\rangle \rightarrow\left|\left\{N_{\bullet}^{\prime}\right\}\right\rangle$, the molecular rotational transitions $|L, M, N\rangle \rightarrow\left|L^{\prime}, M^{\prime}, N^{\prime}\right\rangle$ and the electronic spin transitions $|s, m\rangle \rightarrow\left|s^{\prime}, m^{\prime}\right\rangle$ that satisfy the selection rules (201), (232), (255), and (258) can be represented in terms of diagrams. The horizontal axis oriented from left to right represents the time axis. There are seven 
types of diagram elements:

1. Initial vibration state

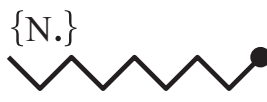

2. Final vibration state

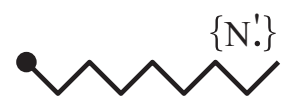

3. Initial rotation state

$\mathrm{M}$ or $\mathrm{N}$

4. Final rotation state

5. Initial spin state

6. Final spin state

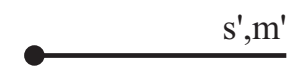

B

7. Bath B

\section{B. Vibrational transitions}

The interaction between the vibrational modes of the system $A$ and the bath $B$ is described by the deformation Hamiltonian $H_{\text {def }}$. According to the expression (131) of $H_{\text {def }}$, the vibrational fluctuation and dissipation coefficients are given by

$$
\begin{aligned}
\Gamma_{s\left\{N_{\bullet}^{\prime \prime}\right\}\left\{N_{\bullet}^{\prime \prime \prime}\right\}}^{s\left\{N_{\bullet}\right\}}= & \sum_{\alpha=1}^{3 N-6} \chi_{\alpha \alpha^{\prime}}^{s}\left(\omega_{\left\{N_{\bullet}\right\}\left\{N_{\bullet}^{\prime \prime}\right\}}^{s}\right) \\
& \times\left\langle\left\{N_{\bullet}^{\prime}\right\}\left|Q_{\alpha^{\prime}}^{s}\right|\left\{N_{\bullet}^{\prime \prime \prime}\right\}\right\rangle\left\langle\left\{N_{\bullet}^{\prime \prime}\right\}\left|Q_{\alpha}^{s}\right|\left\{N_{\bullet}\right\}\right\rangle,
\end{aligned}
$$

where the susceptibilities are given by

$$
\begin{aligned}
\chi_{\alpha \alpha^{\prime}}^{s}\left(\omega_{\left\{N_{\bullet}\right\}\left\{N_{\bullet}^{\prime \prime}\right\}}^{s}\right)= & \frac{2 \pi}{\hbar^{2}} \sum_{b, b^{\prime \prime}} p_{b}^{\tilde{s}} \delta\left(\omega_{\left\{N_{\bullet}\right\}\left\{N_{\bullet}^{\prime \prime}\right\}}^{s}+\omega_{b b^{\prime \prime}}^{\tilde{s} \tilde{s}}\right) \\
& \times\left\langle\tilde{s}, b\left|\tilde{V}_{\alpha^{\prime}}^{\tilde{s}}\right| \tilde{s}, b^{\prime \prime}\right\rangle\left\langle\tilde{s}, b^{\prime \prime}\left|\tilde{V}_{\alpha}^{\tilde{s}}\right| \tilde{s}, b\right\rangle
\end{aligned}
$$

if and only if the transition angular frequencies of the system $A$ are equal, i.e., $\omega_{\left\{N_{\bullet}\right\}\left\{N_{\circ}^{\prime \prime}\right\}}^{s}=\omega_{\left\{N_{0}\right\}\left\{N_{\bullet}^{\prime \prime \prime}\right\}}^{s}$; otherwise, the vibrational fluctuation and dissipation coefficients vanish. According to the vibrational selection rules (201), we have

$$
\left\{N_{\bullet}^{\prime \prime}\right\}=\left\{N_{\bullet} \pm \delta_{\bullet}^{\alpha}\right\} \quad \text { and } \quad\left\{N_{\bullet}^{\prime \prime \prime}\right\}=\left\{N_{\bullet}^{\prime} \pm \delta_{\bullet}^{\alpha^{\prime}}\right\} .
$$

The matrix elements (198) and the condition (200) imply that the vibrational fluctuation and dissipation coefficients (276) reduce to

$$
\Gamma_{s\left\{N_{\bullet} \pm \delta_{\bullet}^{\alpha}\right\}\left\{N_{\bullet}^{\prime} \pm \delta_{\bullet}^{\left.\alpha^{\prime}\right\}}\right.}^{s\left\{N_{\bullet}\right\}}=c_{ \pm}^{\omega_{\alpha} N_{\alpha}} c_{ \pm}^{\omega_{\alpha^{\prime}} N_{\alpha^{\prime}}^{\prime}} \chi_{\alpha \alpha^{\prime}}^{s}\left(\omega_{\left\{N_{\bullet}\right\}\left\{N_{\bullet} \pm \delta_{\bullet}^{\alpha}\right\}}^{s}\right) .
$$

According to the definition (109), the transition rate between the vectors $\left|\left\{N_{\bullet}\right\}\right\rangle$ and $\left|\left\{N_{\bullet} \pm \delta_{\bullet}^{\alpha}\right\}\right\rangle$ is given by

$$
\Gamma_{\left\{N_{\bullet}\right\} \rightarrow\left\{N_{\bullet} \pm \delta_{\bullet}^{\alpha}\right\}}^{s \rightarrow s}=\left(c_{ \pm}^{\omega_{\alpha} N_{\alpha}}\right)^{2} \chi_{\alpha \alpha}^{s}\left(\omega_{\left\{N_{\bullet}\right\}\left\{N_{\bullet} \pm \delta_{\bullet}^{\alpha}\right\}}^{s}\right) .
$$

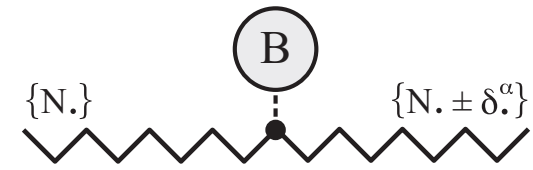

FIG. 3. Diagram of a vibrational state in the mode $\alpha$ interacting with the bath $B$.

The transition described by the transition rate $\Gamma_{\left\{N_{0}\right\} \rightarrow\left\{N_{\bullet} \pm \delta_{\bullet}^{\alpha}\right\}}^{s \rightarrow}$ is represented on the diagram in Fig. 3. The coefficient $c_{ \pm}^{\omega_{\alpha} N_{\alpha}}$ in the transition rate (279) accounts for the initial or final vibration state and the susceptibility $\chi_{\alpha \alpha}^{s}\left(\omega_{\left\{N_{\bullet}\right\}\left\{N_{\bullet} \pm \delta_{\bullet}^{\alpha}\right\}}^{s}\right)$ accounts for the interaction with the bath.

\section{Rotational transitions}

The interaction between the rotational modes of the system $A$ and the bath $B$ is described by the rotational Hamiltonian $H_{\text {ori }}$. According to the first-order term in the expression (131) of $H_{\text {ori }}$, the first kind of rotational fluctuation and dissipation coefficients are given by

$$
\begin{aligned}
\Gamma_{s M^{\prime \prime} M^{\prime \prime \prime}}^{s M M^{\prime}}= & \frac{1}{\hbar^{2}} \chi_{ \pm}^{s}\left(\omega_{M M^{\prime \prime}}^{s s}\left\langle L, M^{\prime}, N\left|L_{\mp}\right| L, M^{\prime \prime \prime}, N\right\rangle\right. \\
& \times\left\langle L, M^{\prime \prime}, N\left|L_{ \pm}\right| L, M, N\right\rangle,
\end{aligned}
$$

where the susceptibilities are given by

$$
\begin{aligned}
\chi_{ \pm}^{s}\left(\omega_{M M^{\prime \prime}}^{s s}\right)= & 2 \pi \sum_{b, b^{\prime \prime}} p_{b}^{\tilde{s}} \delta\left(\omega_{M M^{\prime \prime}}^{s s}+\omega_{b b^{\prime \prime}}^{\tilde{s} \tilde{s}}\right) \\
& \times\left\langle\tilde{s}, b\left|\tilde{\Omega}^{\mp}\right| \tilde{s}^{\prime}, b^{\prime \prime}\right\rangle\left\langle\tilde{s}^{\prime}, b^{\prime \prime}\left|\tilde{\Omega}^{ \pm}\right| \tilde{s}, b\right\rangle
\end{aligned}
$$

if and only if the transition angular frequencies of the system $A$, are equal, i.e., $\omega_{M M^{\prime \prime}}^{s s}=\omega_{M^{\prime} M^{\prime \prime \prime}}^{s s}$; otherwise, the rotational fluctuation and dissipation coefficients vanish. According to the rotational selection rule (232), we have

$$
M^{\prime \prime}=M \pm 1 \quad \text { and } \quad M^{\prime \prime \prime}=M^{\prime} \pm 1
$$

The matrix elements (231) and the condition (229) imply that the rotational fluctuation and dissipation coefficients (280) reduce to

$$
\Gamma_{s M \pm 1 M^{\prime} \pm 1}^{s M}=c_{ \pm}^{L M} c_{ \pm}^{L^{\prime} M^{\prime}} \chi_{ \pm}^{s}\left(\omega_{M M \pm 1}^{s s}\right) .
$$

According to the definition (109), the transition rate between the vectors $|L, M, N\rangle$ and $|L, M \pm 1, N\rangle$ is given by

$$
\Gamma_{M \rightarrow M \pm 1}^{s \rightarrow s}=\left(c_{ \pm}^{L M}\right)^{2} \chi_{ \pm}^{s}\left(\omega_{M M \pm 1}^{s s}\right) .
$$

The transition described by the transition rate $\Gamma_{M \rightarrow M \pm 1}^{s \rightarrow s}$ is represented on the diagram in Fig. 4. The coefficient $c_{ \pm}^{L M}$ in the transition rate (283) accounts for the initial or final rotation state and the susceptibility $\chi_{ \pm}^{s}\left(\omega_{M M \pm 1}^{s s}\right)$ accounts for the interaction with the bath.

The second kind of rotational fluctuation and dissipation coefficients are given by interchanging $M \leftrightarrow N$ and $L_{ \pm} \leftrightarrow \tilde{L}_{ \pm}$

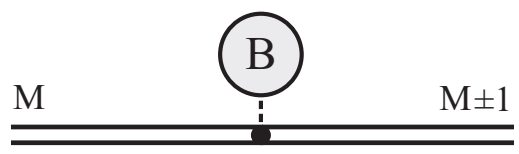

FIG. 4. Diagram of a rotational state interacting with the bath $B$. 


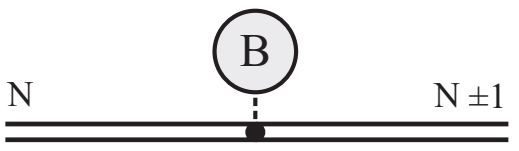

FIG. 5. Diagram of a rotational state interacting with the bath $B$.

and $\tilde{\Omega}^{ \pm} \leftrightarrow \Omega^{ \pm}$in expressions (280) and (281),

$$
\begin{aligned}
\Gamma_{s N^{\prime \prime} N^{\prime \prime \prime}}^{s N N^{\prime}}= & \frac{1}{\hbar^{2}} \chi_{ \pm}^{s}\left(\omega_{N N^{\prime \prime}}^{s s}\right)\left\langle L, M, N^{\prime}\left|\tilde{L}_{\mp}\right| L, M, N^{\prime \prime \prime}\right\rangle \\
& \times\left\langle L, M, N^{\prime \prime}\left|\tilde{L}_{ \pm}\right| L, M, N\right\rangle,
\end{aligned}
$$

where the susceptibilities are given by

$$
\begin{aligned}
\chi_{ \pm}^{s}\left(\omega_{N N^{\prime \prime}}^{s s}\right)= & 2 \pi \sum_{b, b^{\prime \prime}} p_{b}^{\tilde{s}} \delta\left(\omega_{N N^{\prime \prime}}^{s s}+\omega_{b b^{\prime \prime}}^{\tilde{s} \tilde{s}}\right) \\
& \times\left\langle\tilde{s}, b\left|\Omega^{\mp}\right| \tilde{s}^{\prime}, b^{\prime \prime}\right\rangle\left\langle\tilde{s}^{\prime}, b^{\prime \prime}\left|\Omega^{ \pm}\right| \tilde{s}, b\right\rangle
\end{aligned}
$$

if and only if the transition angular frequencies of the system $A$, are equal, i.e., $\omega_{N N^{\prime \prime}}^{s s}=\omega_{N^{\prime} N^{\prime \prime}}^{s s}$; otherwise, the rotational fluctuation and dissipation coefficients vanish. According to the rotational selection rule (232), we have

$$
N^{\prime \prime}=N \pm 1 \quad \text { and } \quad N^{\prime \prime \prime}=N^{\prime} \pm 1 .
$$

The matrix elements (231) and the condition (229) imply that the rotational fluctuation and dissipation coefficients (284) reduce to

$$
\Gamma_{s N \pm 1 N^{\prime} \pm 1}^{s N} \underset{N^{\prime}}{S}=c_{ \pm}^{L N} c_{ \pm}^{L^{\prime} N^{\prime}} \chi_{ \pm}^{s}\left(\omega_{N N \pm 1}^{s s}\right) .
$$

According to the definition (109), the transition rate between the vectors $|L, M, N\rangle$ and $|L, M, N \pm 1\rangle$ is given by

$$
\Gamma_{N \rightarrow N \pm 1}^{s \rightarrow s}=\left(c_{ \pm}^{L N}\right)^{2} \chi_{ \pm}^{s}\left(\omega_{N N \pm 1}^{s s}\right) .
$$

The transition described by the transition rate $\Gamma_{N \rightarrow N \pm 1}^{s \rightarrow s}$ is represented on the diagram in Fig. 5. The coefficient $c_{ \pm}^{L N}$ in the transition rate (287) accounts for the initial or final rotation state and the susceptibility $\chi_{ \pm}^{s}\left(\omega_{N N \pm 1}^{s s}\right)$ accounts for the interaction with the bath.

\section{Spin transitions}

The interaction between the spin properties of the system $A$ and the bath $B$ is described by the spin-orbit Hamiltonian $H_{\text {SO }}$. According to the first-order term in the expression (163) of the Hamiltonians $H_{\mathrm{SO}}^{[n]}$ and $H_{\mathrm{SO}}^{[n-1,1]}$, the spin fluctuation and dissipation coefficients are given by

$$
\begin{aligned}
\Gamma_{s^{\prime} m^{\prime \prime} m^{\prime \prime \prime}}^{s m m^{\prime}}= & \sum_{\mu} \frac{1}{\hbar^{2}} \chi_{k}^{s \mu s^{\prime}}\left(\omega_{m m^{\prime \prime}}^{s s^{\prime}}\right) \\
& \times\left\langle s, m^{\prime}\left|s_{k^{*}}^{s \mu s^{\prime}}\right| s^{\prime}, m^{\prime \prime \prime}\right\rangle\left\langle s^{\prime}, m^{\prime \prime}\left|s_{k}^{s^{\prime} \mu s}\right| s, m\right\rangle,
\end{aligned}
$$

where $k \in\{-,+, 3\}$, the isotypic components of types $\mu \in$ $\{[n],[n-1,1]\}$, and the susceptibilities are given by

$$
\begin{aligned}
\chi_{k}^{s \mu s^{\prime}}\left(\omega_{m m^{\prime \prime}}^{s s^{\prime}}\right)= & 2 \pi\left(g^{s^{\prime} \mu s}\right)^{2} \sum_{b, b^{\prime \prime}} p_{b}^{\tilde{s}} \delta\left(\omega_{m m^{\prime \prime}}^{s s^{\prime}}+\omega_{b b^{\prime \prime}}^{\tilde{s} \tilde{s}^{\prime}}\right) \\
& \times\left\langle\tilde{s}, b\left|\tilde{B}^{\tilde{s} \mu^{\prime} \tilde{s}^{\prime} k^{*}}\right| \tilde{s}^{\prime}, b^{\prime \prime}\right\rangle\left\langle\tilde{s}^{\prime}, b^{\prime \prime}\left|\tilde{B}^{\tilde{s}^{\prime} \mu \tilde{s} k}\right| \tilde{s}, b\right\rangle
\end{aligned}
$$

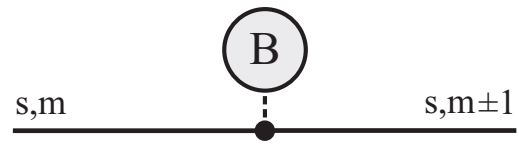

FIG. 6. Diagram of a spin state interacting with the bath $B$.

if and only if the transition angular frequencies of the system $A$, are equal, i.e., $\omega_{m m^{\prime \prime}}^{s s^{\prime}}=\omega_{m^{\prime} m^{\prime \prime \prime}}^{s s^{\prime}}$; otherwise, the spin fluctuation and dissipation coefficients vanish. According to the spin selection rule (258), we have

$$
m^{\prime \prime}=m \pm 1 \quad \text { and } \quad m^{\prime \prime \prime}=m^{\prime} \pm 1 .
$$

If the spin quantum numbers are equal, i.e., $s^{\prime}=s$, the spin fluctuation and dissipation coefficients (288) become

$$
\begin{aligned}
\Gamma_{s m^{\prime \prime} m^{\prime \prime \prime}}^{s m m m^{\prime}}= & \frac{1}{\hbar^{2}} \chi_{ \pm}^{s[n] s}\left(\omega_{m m^{\prime \prime}}^{s s}\right) \\
& \times\left\langle s, m^{\prime}\left|s_{\mp}^{s[n] s}\right| s, m^{\prime \prime \prime}\right\rangle\left\langle s, m^{\prime \prime}\left|s_{ \pm}^{s[n] s}\right| s, m\right\rangle .
\end{aligned}
$$

The matrix elements (264) and the conditions (250) imply that the spin fluctuation and dissipation coefficients (290) reduce to

$$
\Gamma_{s m \pm 1 m^{\prime} \pm 1}^{s m}=c_{ \pm}^{s m} c_{ \pm}^{s m^{\prime}} \chi_{ \pm}^{s[n] s}\left(\omega_{m m \pm 1}^{s s}\right)
$$

According to the definition (109), the transition rate between the vectors $|s, m\rangle$ and $|s, m \pm 1\rangle$ is given by

$$
\Gamma_{m \rightarrow m \pm 1}^{s \rightarrow s}=\left(c_{ \pm}^{s m}\right)^{2} \chi_{ \pm}^{s[n] s}\left(\omega_{m m \pm 1}^{s s}\right) .
$$

The transition described by the transition rate $\Gamma_{m \rightarrow m \pm 1}^{s \rightarrow s}$ is represented on the diagram in Fig. 6. The coefficient $c_{ \pm}^{s m}$ in the transition rate (292) accounts for the initial or final spin state and the susceptibility $\chi_{ \pm}^{s[n] s}\left(\omega_{m m \pm 1}^{s s}\right)$ accounts for the interaction with the bath.

According to the spin selection rule (255), if the spin quantum numbers are different, we have $s^{\prime}=s \pm 1$. In this case, the spin fluctuation and dissipation coefficients (288) are recast as

$$
\begin{aligned}
\Gamma_{s \pm 1 m^{\prime \prime} m^{\prime \prime \prime}}^{s m m^{\prime}}= & \frac{1}{\hbar^{2}} \chi_{k}^{s[n-1,1] s \pm 1 k}\left(\omega_{m m^{\prime \prime}}^{s s \pm 1}\right) \\
& \times\left\langle s, m^{\prime}\left|s_{k^{*}}^{s[n-1,1] s \pm 1}\right| s \pm 1, m^{\prime \prime \prime}\right\rangle \\
& \times\left\langle s \pm 1, m^{\prime \prime}\left|s_{k}^{s \pm 1[n-1,1] s}\right| s, m\right\rangle .
\end{aligned}
$$

The matrix elements (268), (269), (272), and (273) imply that the spin fluctuation and dissipation coefficients (293) reduce to

$$
\begin{aligned}
\Gamma_{s \pm 1 m \pm 1 m^{\prime} \pm 1}^{s m} & =c_{s \pm 1 m \pm 1}^{s m} c_{s \pm 1 m^{\prime} \pm 1}^{s m^{\prime}} \chi_{ \pm}^{s[n-1, n] s \pm 1}\left(\omega_{m m \pm 1}^{s s \pm 1}\right), \\
\Gamma_{s \pm 1 m \mp 1 m^{\prime} \mp 1}^{s m} & =c_{s \pm 1 m \mp 1}^{s m} c_{s \pm 1 m^{\prime} \mp 1}^{s m^{\prime}} \chi_{\mp}^{s[n-1, n] s \pm 1}\left(\omega_{m m \mp 1}^{s s \pm 1}\right), \\
\Gamma_{s \pm 1 m m^{\prime}}^{s m m^{\prime}} & =c_{s \pm 1 m}^{s m} c_{s \pm 1 m^{\prime}}^{s m^{\prime}} \chi_{3}^{s[n-1, n] s \pm 1}\left(\omega_{m m}^{s s \pm 1}\right) .
\end{aligned}
$$

According to definition (109), the transition rates between the vectors $|s, m\rangle$ and $|s \pm 1, m \pm 1\rangle$, between the vectors $|s, m\rangle$ and $|s \pm 1, m \mp 1\rangle$, and between the vectors $|s, m\rangle$ and $|s \pm 1, m\rangle$ 


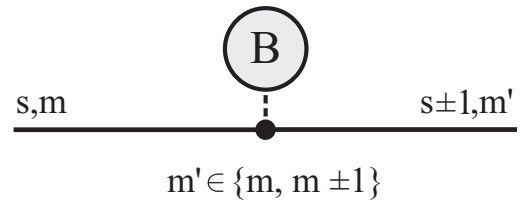

FIG. 7. Diagram of a spin state interacting with the bath $B$.

are given by

$$
\begin{aligned}
\Gamma_{m \rightarrow m \pm 1}^{s \rightarrow s \pm 1} & =\left(c_{s \pm 1 m \pm 1}^{s m}\right)^{2} \chi_{ \pm}^{s[n-1, n] s \pm 1}\left(\omega_{m m \pm 1}^{s s \pm 1}\right), \\
\Gamma_{m \rightarrow m \mp 1}^{s \rightarrow s \pm 1} & =\left(c_{s \pm 1 m \mp 1}^{s m}\right)^{2} \chi_{\mp}^{s[n-1, n] s \pm 1}\left(\omega_{m m \mp 1}^{s s \pm 1}\right), \\
\Gamma_{m \rightarrow s}^{s \rightarrow 1} & =\left(c_{s \pm 1 m}^{s m}\right)^{2} \chi_{3}^{s[n-1, n] s \pm 1}\left(\omega_{m m}^{s s \pm 1}\right) .
\end{aligned}
$$

The transition rates $\Gamma_{m \rightarrow m \pm 1}^{s \rightarrow s \pm 1}, \quad \Gamma_{m \rightarrow m \mp 1}^{s \rightarrow s \pm 1}$, and $\Gamma_{m \rightarrow m}^{s \rightarrow s \pm 1}$ are represented in the diagram in Fig. 7. The coefficients $c_{s \pm 1 m \pm 1}^{s m}, c_{s \pm 1 m \mp 1}^{s m}$, and $c_{s \pm 1 m}^{s m}$ in the transition rates (295) account for the initial or final spin states and the susceptibilities $\chi_{ \pm}^{s[n-1, n] s \pm 1}\left(\omega_{m m \pm 1}^{s s \pm 1}\right), \quad \chi_{\mp}^{s[n-1, n] s \pm 1}\left(\omega_{m m \mp 1}^{s s \pm 1}\right)$, and $\chi_{3}^{s[n-1, n] s \pm 1}\left(\omega_{m m}^{s s \pm 1}\right)$ account for the interaction with the bath.

\section{E. Matrix elements of the external Hamiltonian}

According to the expression (166) of the Hamiltonian $H_{\text {ext }}(t)$, the matrix elements (105) are recast as

$$
\begin{aligned}
& H_{\operatorname{ext}^{\prime} M^{\prime} M}^{s}(t)=-\left\langle L, M^{\prime}, N\left|L_{+}\right| L, M^{\prime}-1, N\right\rangle \\
& \times\left\langle L, M^{\prime}-1, N\left|\Omega_{\mathrm{ext}}^{-}(t)\right| L, M, N\right\rangle \\
& -\left\langle L, M^{\prime}, N\left|L_{-}\right| L, M^{\prime}+1, N\right\rangle \\
& \times\left\langle L, M^{\prime}+1, N\left|\Omega_{\mathrm{ext}}^{+}(t)\right| L, M, N\right\rangle \\
& -\left\langle L, M^{\prime}, N\left|L_{3}\right| L, M^{\prime}, N\right\rangle \\
& \times\left\langle L, M^{\prime}, N\left|\Omega_{\mathrm{ext}}^{3}(t)\right| L, M, N\right\rangle, \\
& H_{\operatorname{ext}_{M^{\prime} M}{ }^{s}}(t)=-\left\langle L, M, N^{\prime}\left|\tilde{L}_{+}\right| L, M, N^{\prime}-1\right\rangle \\
& \times\left\langle L, M, N^{\prime}-1\left|\tilde{\Omega}_{\mathrm{ext}}^{-}(t)\right| L, M, N\right\rangle \\
& -\left\langle L, M, N^{\prime}\left|\tilde{L}_{-}\right| L, M, N^{\prime}+1\right\rangle \\
& \times\left\langle L, M, N^{\prime}+1\left|\tilde{\Omega}_{\mathrm{ext}}^{+}(t)\right| L, M, N\right\rangle \\
& -\left\langle L, M, N^{\prime}\left|\tilde{L}_{3}\right| L, M, N^{\prime}\right\rangle \\
& \times\left\langle L, M, N^{\prime}\left|\tilde{\Omega}_{\mathrm{ext}}^{3}(t)\right| L, M, N\right\rangle, \\
& -\gamma_{e}\left\langle s, m^{\prime}\left|s_{-}\right| s, m^{\prime}+1\right\rangle\left\langle s, m^{\prime}+1\left|B_{\mathrm{ext}}^{+}(t)\right| s, m\right\rangle \\
& -\gamma_{e}\left\langle s, m^{\prime}\left|s_{3}\right| s, m^{\prime}\right\rangle\left\langle s, m^{\prime}\left|B_{\mathrm{ext}}^{3}(t)\right| s, m\right\rangle,
\end{aligned}
$$

where

$$
\begin{aligned}
& \Omega_{\mathrm{ext}}^{ \pm}(t)=\frac{1}{2}\left[\Omega_{\mathrm{ext}}^{1}(t) \pm i \Omega_{\mathrm{ext}}^{2}(t)\right], \\
& \tilde{\Omega}_{\mathrm{ext}}^{ \pm}(t)=\frac{1}{2}\left[\tilde{\Omega}_{\mathrm{ext}}^{1}(t) \pm i \tilde{\Omega}_{\mathrm{ext}}^{2}(t)\right], \\
& B_{\mathrm{ext}}^{ \pm}(t)=\frac{1}{2}\left[B_{\mathrm{ext}}^{1}(t) \pm i B_{\mathrm{ext}}^{2}(t)\right] .
\end{aligned}
$$

The matrix elements appearing in the relations (296), (297), and (298) are expressed explicitly as

$$
\begin{aligned}
\Omega_{\mathrm{ext} M^{\prime} \pm 1 M}^{ \pm}(t) & \equiv\left\langle L, M^{\prime} \pm 1, N\left|\Omega_{\mathrm{ext}}^{ \pm}(t)\right| L, M, N\right\rangle, \\
\Omega_{\operatorname{ext} M^{\prime} M}^{3 s}(t) & \equiv\left\langle L, M^{\prime}, N\left|\Omega_{\mathrm{ext}}^{ \pm}(t)\right| L, M, N\right\rangle,
\end{aligned}
$$

$$
\begin{aligned}
\tilde{\Omega}_{\mathrm{ext} N^{\prime} \pm 1 N}^{ \pm}(t) & \equiv\left\langle L, M, N^{\prime} \pm 1\left|\tilde{\Omega}_{\mathrm{ext}}^{ \pm}(t)\right| L, M, N\right\rangle, \\
\tilde{\Omega}_{\mathrm{ext} N^{\prime} N}^{3 s}(t) & \equiv\left\langle L, M, N^{\prime}\left|\tilde{\Omega}_{\mathrm{ext}}^{ \pm}(t)\right| L, M, N\right\rangle, \\
B_{\mathrm{ext} m^{\prime} \pm 1 m}^{ \pm s}(t) & \equiv\left\langle s, m^{\prime} \pm 1\left|B_{\mathrm{ext}}^{ \pm}(t)\right| s, m\right\rangle, \\
B_{\mathrm{ext} m^{\prime} m}^{3 s}(t) & \equiv\left\langle s, m^{\prime}\left|B_{\mathrm{ext}}^{3}(t)\right| s, m\right\rangle .
\end{aligned}
$$

Using the expressions (226), (228), (247), (248), and (300), the matrix elements (296), (297), and (298) are recast, respectively, as, recast as

$$
\begin{aligned}
H_{\operatorname{ext}^{M^{\prime} M}}^{s}(t)= & -\hbar M \Omega_{\operatorname{ext} M^{\prime} M}^{-s}(t)-\hbar c_{+}^{L^{\prime} M^{\prime}-1} \Omega_{\operatorname{ext} M^{\prime}-1 M}^{-s}(t) \\
& -\hbar c_{-}^{L^{\prime} M^{\prime}+1} \Omega_{\operatorname{ext} M^{\prime}+1 M}^{+s}(t) \\
H_{\operatorname{ext}_{N^{\prime} N}^{s}(t)=} & -\hbar N \Omega_{\operatorname{ext} N^{\prime} N}^{-s}(t)-\hbar c_{+}^{L^{\prime} N^{\prime}-1} \tilde{\Omega}_{\operatorname{ext} N^{\prime}-1 N}^{-s}(t) \\
& -\hbar c_{-}^{L^{\prime} N^{\prime}+1} \tilde{\Omega}_{\operatorname{ext} N^{\prime}+1 N}^{+s}(t) \\
H_{\operatorname{ext}{ }_{m^{\prime} m}^{s}}(t)= & -\gamma_{e} \hbar m B_{\operatorname{ext} m^{\prime} m}^{-s}(t)-\gamma_{e} \hbar c_{+}^{s^{\prime} m^{\prime}-1} B_{\operatorname{ext} m^{\prime}-1 m}^{-s}(t) \\
& -\gamma_{e} \hbar c_{-}^{s^{\prime} m^{\prime}+1} B_{\operatorname{ext} m^{\prime}+1 m}^{+s}(t)
\end{aligned}
$$

\section{CONCLUSION}

The relaxation processes involving vibrational and rotational properties, as well as electronic spin properties in a midsize molecule, are expressed by the quantum master equations where the bath consists of the electronic spatial properties. The master equations allow for the presence of an external magnetic field and more generally of an electromagnetic field.

The evolution of the diagonal and off-diagonal matrix elements of the density operator, obtained from the master equation, describe the evolution of the populations and coherences, respectively. The evolution equations for the populations and the coherences are coupled in the presence of an external field. These equations are written in terms of the fluctuation and dissipation coefficients $\Gamma_{s^{\prime} a^{\prime \prime} a^{\prime \prime \prime}}^{s a}$. These coefficients are expressed, in turn, as the product of coefficients entirely determined by the symmetries associated with the vibrational, rotational, and spin properties, i.e., $\left\{N_{\bullet}^{\prime}\right\}, L, M, N, s, m$, and of the susceptibilities describing the response of the bath to the polarization induced by the system. The susceptibilities of the bath are functions of the transitions frequencies only. They can be treated phenomenologically.

In our quantum description of a molecule, the orientation is treated as a genuine quantum property characterized by a pseudovectorial operator $\omega \in \mathcal{L}\left(\mathcal{H}_{\text {ori }}\right)$. The orbital angular momentum operator and the orientation operator satisfy canonical commutation relations [30]. In contrast to a classical approach, in a quantum approach no reference frame can be attached to the molecule since the molecular orientation is not an "actual" property $[43,44]$.

This work provides a basis for the study of the linear response of a molecule to an external magnetic field. In a quantum description of a rotating molecule, the Zeeman coupling with the external field is expected to lead to nontrivial effects since the spins are rotating with the molecule.

Relaxation processes involving the nuclear spin are currently widely studied in the field of dynamic nuclear polarization [45]. Such processes can be treated by including the nuclear spin properties in the description of the system. 
Electromagnetic fields also play an important role in molecular relaxation processes $[46,47]$. Therefore, it will also be interesting to include the electromagnetic fields in the quantum description of the system. This will allow for the discussion of spontaneous and induced emissions and it will help to understand the spectroscopic observations performed within molecular relaxation experiments [3].

\section{APPENDIX A: ROTATION OF THE OPERATOR $\boldsymbol{s}_{v}$}

In this appendix, we establish the explicit expression for the rotation of the operator $s_{\nu}$, which is treated here as a dimensionless operator. The action of the rotation group $\mathrm{SO}(3)$ on the Hilbert space $\mathcal{H}_{A}^{s}$ associated with the spin of a single electron is given by the unitary representation $\omega \mapsto U_{A}(\omega)$, where the orientation operator $\omega \in \mathcal{L}\left(\mathcal{H}_{\text {ori }}\right)$ and the unitary operator $U_{A}(\omega) \in \mathcal{L}\left(\mathcal{H}_{s}^{\otimes n}\right)$. The unitary operator $U_{A}(\omega)$ is expressed as

$$
U_{A}(\omega)=\exp \left(-\frac{i}{2} \omega \cdot \sigma\right)
$$

where $\sigma$ is the Pauli vector that satisfies the commutation relation,

$$
\left[\frac{i}{2} \omega \cdot \sigma, \sigma\right]=\omega \times \sigma .
$$

The operators $s_{v}(\omega)$ are the conjugates of the operators $s_{v}$ with respect to the unitary operator $U_{A}(\omega)$, i.e.,

$$
\boldsymbol{s}_{\nu}(\boldsymbol{\omega})=U_{A}(\boldsymbol{\omega}) \boldsymbol{s}_{\nu} U_{A}(\boldsymbol{\omega})^{-1}
$$

The operators $\boldsymbol{s}_{v}$ are expressed in terms of the Pauli vector $\boldsymbol{\sigma}$ as

$$
\boldsymbol{s}_{v}=\mathbb{1} \otimes \underbrace{\ldots}_{2, \ldots, v-1} \otimes \frac{1}{2} \boldsymbol{\sigma} \otimes \underbrace{\ldots}_{\nu+1, \ldots, n-1} \otimes \mathbb{1} .
$$

Using the definitions (A1) and (A4), relation (A3) is recast as

$$
\begin{aligned}
U_{A}(\boldsymbol{\omega}) \boldsymbol{s}_{\nu} U_{A}(\boldsymbol{\omega})^{-1}= & \mathbb{1} \otimes \underbrace{\ldots}_{2, \ldots, \nu-1} \otimes \exp \left(-\frac{i}{2} \boldsymbol{\omega} \cdot \boldsymbol{\sigma}\right) \frac{1}{2} \boldsymbol{\sigma} \\
& \times \exp \left(\frac{i}{2} \boldsymbol{\omega} \cdot \boldsymbol{\sigma}\right) \otimes \underbrace{\ldots}_{\nu+1, \ldots, n-1} \otimes \mathbb{1} .
\end{aligned}
$$

Moreover, using the commutation relation (A2) and the BakerCampbell-Hausdorff formula, we obtain the identity

$$
\begin{aligned}
& \exp \left(-\frac{i}{2} \boldsymbol{\omega} \cdot \boldsymbol{\sigma}\right) \boldsymbol{\sigma} \exp \left(\frac{i}{2} \boldsymbol{\omega} \cdot \boldsymbol{\sigma}\right) \\
& =\sum_{k=0}^{\infty} \frac{(-1)^{k}}{k !}\left[\frac{i}{2} \boldsymbol{\omega} \cdot \boldsymbol{\sigma}, \boldsymbol{\sigma}\right]_{k}=\sum_{k=0}^{\infty} \frac{(-1)^{k}}{k !}(\boldsymbol{\omega} \times \boldsymbol{\sigma})_{k},
\end{aligned}
$$

where the nested commutators and vectorial products of operators are defined, respectively, as

$$
[\boldsymbol{X}, \boldsymbol{Y}]_{0}=\boldsymbol{Y}, \quad[\boldsymbol{X}, \boldsymbol{Y}]_{k}=[\boldsymbol{X},[\boldsymbol{X}, \boldsymbol{Y}]]_{k-1},
$$

and

$$
(\boldsymbol{X} \times \boldsymbol{Y})_{0}=\boldsymbol{Y}, \quad(\boldsymbol{X} \times \boldsymbol{Y})_{k}=(\boldsymbol{X} \times(\boldsymbol{X} \times \boldsymbol{Y}))_{k-1} .
$$

The projector on the orientation operator $\omega$ is defined as

$$
\mathcal{P}_{\omega}=\frac{\omega \omega}{\omega \cdot \omega} \equiv \hat{\omega} \hat{\omega}
$$

where $\hat{\omega}=\omega / \omega$ and $\omega=\|\omega\|$. Using the properties

$$
\begin{aligned}
(\boldsymbol{\omega} \times \boldsymbol{\sigma})_{2} & =\boldsymbol{\omega} \times(\boldsymbol{\omega} \times \boldsymbol{\sigma})=-n^{2} \boldsymbol{\sigma}+\boldsymbol{\omega}(\boldsymbol{\omega} \cdot \boldsymbol{\sigma}) \\
& =-n^{2}\left(\mathbb{1}-\mathcal{P}_{\omega}\right) \boldsymbol{\sigma}, \\
\mathcal{P}_{\omega}^{2} & =\mathcal{P}_{\omega} \Rightarrow\left(\mathbb{1}-\mathcal{P}_{\omega}\right)^{k}=\mathbb{1}-\mathcal{P}_{\omega} \quad \forall k \in \mathbb{N}^{\star}, \\
\mathcal{P}_{\omega}(\boldsymbol{\omega} \times \boldsymbol{\sigma}) & =\frac{\omega \cdot(\boldsymbol{\omega} \times \boldsymbol{\sigma})}{\boldsymbol{\omega} \cdot \boldsymbol{\omega}} \boldsymbol{\omega}=\mathbf{0},
\end{aligned}
$$

the sum of nested vectorial products in the relation (A6) is recast as

$$
\begin{aligned}
& \sum_{k=0}^{\infty} \frac{(-1)^{k}}{k !}(\boldsymbol{\omega} \times \boldsymbol{\sigma})_{n} \\
& =\boldsymbol{\sigma}+\sum_{k=1}^{\infty} \frac{(-1)^{2 k}}{(2 k) !}(\boldsymbol{\omega} \times \boldsymbol{\sigma})_{2 k}-\sum_{k=0}^{\infty} \frac{(-1)^{2 k+1}}{(2 k+1) !}(\boldsymbol{\omega} \times \boldsymbol{\sigma})_{2 k+1} \\
& =\sigma+\sum_{k=1}^{\infty} \frac{(-1)^{2 k}}{(2 k) !} n^{2 k}\left(\mathbb{1}-\mathcal{P}_{\omega}\right)^{k} \boldsymbol{\sigma} \\
& -\sum_{k=0}^{\infty} \frac{(-1)^{2 k+1}}{(2 k+1) !} n^{2 k}\left(\mathbb{1}-\mathcal{P}_{\omega}\right)^{k}(\boldsymbol{\omega} \times \boldsymbol{\sigma}) \\
& =\boldsymbol{\sigma}+\sum_{k=1}^{\infty} \frac{(-1)^{2 k}}{(2 k) !} n^{2 k}\left(\mathbb{1}-\mathcal{P}_{\omega}\right) \boldsymbol{\sigma} \\
& -\frac{1}{n} \sum_{k=0}^{\infty} \frac{(-1)^{2 k+1}}{(2 k+1) !} n^{2 k+1}(\boldsymbol{\omega} \times \boldsymbol{\sigma}) \\
& =\sigma+(\cos \omega-\mathbb{1})\left(\mathbb{1}-\mathcal{P}_{\omega}\right) \sigma-\frac{\sin \omega}{\omega}(\omega \times \sigma) \\
& =\mathcal{P}_{\omega} \cdot \sigma+\left(\mathbb{1}-\mathcal{P}_{\omega}\right) \boldsymbol{\sigma} \cos \omega-(\omega \times \boldsymbol{\sigma}) \frac{\sin \omega}{\omega} \\
& =(\hat{\boldsymbol{\omega}} \cdot \boldsymbol{\sigma}) \hat{\boldsymbol{\omega}}+[\boldsymbol{\sigma}-(\hat{\boldsymbol{\omega}} \cdot \boldsymbol{\sigma}) \hat{\boldsymbol{\omega}}] \cos \omega-(\hat{\boldsymbol{\omega}} \times \boldsymbol{\sigma}) \sin \omega .
\end{aligned}
$$

Using the relations (A3)-(A6) and (A11), the internal operators $\boldsymbol{s}_{v}(\boldsymbol{\omega})$ are expressed explicitly in terms of the operators $\boldsymbol{s}_{v}$ according to

$$
\begin{aligned}
\boldsymbol{s}_{\nu}(\boldsymbol{\omega})= & \left(\hat{\boldsymbol{\omega}} \cdot \boldsymbol{s}_{\nu}\right) \hat{\boldsymbol{\omega}}+\left[\boldsymbol{s}_{\nu}-\left(\hat{\boldsymbol{\omega}} \cdot \boldsymbol{s}_{\nu}\right) \hat{\boldsymbol{\omega}}\right] \cos \omega \\
& -\left(\hat{\boldsymbol{\omega}} \times \boldsymbol{s}_{\nu}\right) \sin \omega .
\end{aligned}
$$

\section{APPENDIX B: MATRIX ELEMENTS OF THE OPERATORS $s_{v 3}$ AND $s_{v \pm}$}

In this appendix, we determine the matrix elements of the operators $s_{\nu 3}$ and $s_{\nu \pm}$, which are treated as dimensionless operators. We consider transitions between an initial vector $|s, m, i\rangle \in \mathcal{H}_{m}^{s}$ and a final vector $\left|s^{\prime}, m^{\prime}, i^{\prime}\right\rangle \in \mathcal{H}_{m^{\prime}}^{s^{\prime}}$. 
The spin selection rules (255) and (258) set restrictions on the matrix elements of the operators $s_{v 3}$ and $s_{v \pm}$, i.e.,

$$
\begin{aligned}
& \left\langle s^{\prime}, m^{\prime}, i^{\prime}\left|s_{\nu 3}\right| s, m, i\right\rangle=0, \\
& \text { if }\left|s^{\prime}-s\right|>1 \text { or }\left|m^{\prime}-m\right| \neq 0, \\
& \left\langle s^{\prime}, m^{\prime}, i^{\prime}\left|s_{\nu \pm}\right| s, m, i\right\rangle=0, \\
& \text { if }\left|s^{\prime}-s\right|>1 \text { or }\left|m^{\prime}-m\right| \neq 1 .
\end{aligned}
$$

Proposition 1. The action of the operators $s_{\nu 3}$ and $s_{v \pm}$ on the eigenvectors $|s, m, i\rangle$ is expressed in terms of the quantum numbers $s$ and $m$ and the operators $\Lambda_{v}^{-}(s-1), \Lambda_{v}^{3}(s)$, and $\Lambda_{v}^{+}(s+1)$ as

$$
\begin{aligned}
s_{\nu 3}|s, m, i\rangle= & \sqrt{(s-m)(s+m)} \Lambda_{v}^{-}(s-1)|s, m, i\rangle \\
& +m \Lambda_{v}^{3}(s)|s, m, i\rangle \\
& +\sqrt{(s-m+1)(s+m+1)} \Lambda_{v}^{+}(s+1)|s, m, i\rangle,
\end{aligned}
$$

$$
\begin{aligned}
s_{v \pm}|s, m, i\rangle= & \pm \sqrt{(s \mp m-1)(s \mp m)} \Lambda_{v}^{-}(s-1)|s, m \pm 1, i\rangle \\
& +\sqrt{(s \mp m)(s \pm m+1)} \Lambda_{v}^{3}(s)|s, m \pm 1, i\rangle \\
& \mp \sqrt{(s \pm m+1)(s \pm m+2)} \\
& \times \Lambda_{v}^{+}(s+1)|s, m \pm 1, i\rangle,
\end{aligned}
$$

where

$$
\begin{aligned}
\Lambda_{v}^{3}(s) & : \mathcal{H}_{m}^{s} \rightarrow \mathcal{H}_{m}^{s} \quad \forall s, m, \\
\Lambda_{v}^{ \pm}(s \pm 1): \mathcal{H}_{m}^{s} & \rightarrow \mathcal{H}_{m}^{s \pm 1} \quad \forall s, m .
\end{aligned}
$$

Proof. Taking into account the selection rules (B1), the action of the operator $s_{v 3}$ on the eigenvector $|s, m, i\rangle$ can be expressed as

$$
\begin{aligned}
s_{\nu 3}|s, m, i\rangle= & \Lambda_{\nu 3}^{-}(s-1, m)|s, m, i\rangle+\Lambda_{\nu 3}^{3}(s, m)|s, m, i\rangle \\
& +\Lambda_{\nu 3}^{+}(s+1, m)|s, m, i\rangle .
\end{aligned}
$$

The operator $s_{v 3}$ is represented as a tridiagonal block matrix acting on the Hilbert space $\mathcal{H}_{m}^{s-1} \oplus \mathcal{H}_{m}^{s} \oplus \mathcal{H}_{m}^{s+1}$,

$$
s_{\nu 3}=\left[\begin{array}{ccc}
\Lambda_{v 3}^{3}(s-1, m) & \Lambda_{\nu 3}^{-}(s-1, m) & 0 \\
\Lambda_{\nu 3}^{+}(s, m) & \Lambda_{\nu 3}^{3}(s, m) & \Lambda_{\nu 3}^{-}(s, m) \\
0 & \Lambda_{\nu 3}^{+}(s+1, m) & \Lambda_{\nu 3}^{3}(s+1, m)
\end{array}\right] .
$$

The Hilbert space $\mathcal{H}_{m}^{s-1} \bigoplus \mathcal{H}_{m}^{s} \bigoplus \mathcal{H}_{m}^{s+1}$ is spanned by the set of vectors $\{|s-1, m, i\rangle \otimes|s+1, m, j\rangle \otimes|s+1, m, k\rangle\}$, where $i=1, \ldots, d_{s-1}, j=1, \ldots, d_{s}$, and $k=1, \ldots, d_{s+1}$. The operators $\Lambda_{\nu 3}^{3}(s, m)$ and $\Lambda_{\nu 3}^{ \pm}(s \pm 1, m)$ satisfy the condition

$$
\begin{aligned}
& \left\langle s^{\prime}, m^{\prime}, i^{\prime}\left|\Lambda_{\nu 3}^{3}(s, m)\right| s, m, i\right\rangle=0, \\
& \left\langle s^{\prime} \pm 1, m^{\prime}, i^{\prime}\left|\Lambda_{\nu 3}^{ \pm}(s \pm 1, m)\right| s, m, i\right\rangle=0,
\end{aligned}
$$

if $s^{\prime} \neq s$ or $m^{\prime} \neq m$. Similarly, taking into account the spin selection rules (B2), the action of the operator $s_{\nu \pm}$ on the eigenvector $|s, m, i\rangle$ can be expressed as

$$
\begin{aligned}
s_{\nu \pm}|s, m, i\rangle= & \Lambda_{\nu \pm}^{-}(s-1, m \pm 1)|s, m \pm 1, i\rangle \\
& +\Lambda_{\nu \pm}^{3}(s, m \pm 1)|s, m \pm 1, i\rangle \\
& +\Lambda_{\nu \pm}^{+}(s+1, m \pm 1)|s, m \pm 1, i\rangle,
\end{aligned}
$$

where the operators $\Lambda_{\nu 3}^{3}(s, m \pm 1)$ and $\Lambda_{\nu 3}^{ \pm}(s \pm 1, m \pm 1)$ satisfy the condition

$$
\begin{aligned}
& \left\langle s^{\prime}, m^{\prime} \pm 1, i^{\prime}\left|\Lambda_{v \pm}^{3}(s, m \pm 1)\right| s, m \pm 1, i\right\rangle=0, \\
& \left\langle s^{\prime} \pm 1, m^{\prime} \pm 1, i^{\prime}\left|\Lambda_{v \pm}^{ \pm}(s \pm 1, m \pm 1)\right| s \pm 1, m \pm 1, i\right\rangle=0,
\end{aligned}
$$

if $s^{\prime} \neq s$ or $m^{\prime} \neq m$. Using the definition $s^{2}=s_{i} s^{i}$, the commutation relation

$$
\left[s_{j}, s_{v k}\right]=i \varepsilon_{i j k} \delta^{i \ell} s_{\nu \ell}
$$

yields the commutation relation

$$
\left[s^{2}, s_{v k}\right]=-i \varepsilon^{\ell i j} \delta_{\ell k}\left\{s_{i}, s_{v j}\right\},
$$

which, in turn, implies that

$$
\begin{aligned}
& {\left[s^{2}, s_{\nu 1}\right]=i\left(\left\{s_{3}, s_{v 2}\right\}-\left\{s_{2}, s_{v 3}\right\}\right),} \\
& {\left[s^{2}, s_{\nu 2}\right]=i\left(\left\{s_{1}, s_{\nu 3}\right\}-\left\{s_{3}, s_{v 1}\right\}\right),} \\
& {\left[s^{2}, s_{\nu 3}\right]=i\left(\left\{s_{2}, s_{v 1}\right\}-\left\{s_{1}, s_{v 2}\right\}\right) .}
\end{aligned}
$$

The definition (242) and the third equation (B12) imply that

$$
\left[s^{2}, s_{3}\right]=0 .
$$

Definitions (238) and (243) and Eqs. (B12) imply that

$$
\begin{aligned}
{\left[s^{2}, s_{v \pm}\right] } & = \pm\left(\left\{s_{3}, s_{v \pm}\right\}-\left\{s_{ \pm}, s_{v 3}\right\}\right), \\
{\left[s^{2}, s_{v 3}\right] } & =\frac{1}{2}\left(\left\{s_{+}, s_{v-}\right\}-\left\{s_{-}, s_{v+}\right\}\right) .
\end{aligned}
$$

Using the commutation relation (241) and the definition (242), the commutation relations (B14) reduce to

$$
\begin{gathered}
{\left[s^{2}, s_{\nu \pm}\right]=2 s_{v \pm} \pm 2 s_{v \pm} s_{3} \mp 2 s_{v 3} s_{ \pm},} \\
{\left[s^{2}, s_{v 3}\right]=2 s_{v 3}+s_{v-} s_{+}-s_{v+} s_{-} .}
\end{gathered}
$$

The commutation relation (B15) yields the eigenvalue equation

$$
\begin{gathered}
s^{2} s_{\nu \pm}|s, m, i\rangle-s_{\nu \pm} s^{2}|s, m, i\rangle-2 s_{v \pm}|s, m, i\rangle \\
\mp 2 s_{\nu \pm} s_{3}|s, m, i\rangle=\mp 2 s_{\nu 3} s_{\nu \pm}|s, m, i\rangle .
\end{gathered}
$$

Substituting the expressions (247), (B5), and (B8) in the eigenvalue equation (B16), we obtain the following conditions for the operators acting on the Hilbert spaces $\mathcal{H}_{A m \pm 1}^{s-1}, \mathcal{H}_{A m \pm 1}^{s}$, and $\mathcal{H}_{A m \pm 1}^{s+1}$, respectively,

$$
\begin{gathered}
-2(s \pm m+1) \Lambda_{v \pm}^{-}(s-1, m \pm 1) \\
=\mp 2 c_{ \pm}^{s m} \Lambda_{\nu 3}^{-}(s-1, m \pm 1), \\
\mp 2(m \pm 1) \Lambda_{v \pm}^{3}(s, m \pm 1)=\mp 2 c_{ \pm}^{s m} \Lambda_{v 3}^{3}(s, m \pm 1) \\
2(s \mp m) \Lambda_{v \pm}^{+}(s+1, m \pm 1)=\mp 2 c_{ \pm}^{s m} \Lambda_{v 3}^{+}(s+1, m \pm 1) . \\
\text { Equations }(\mathrm{B} 17)-(\mathrm{B} 19) \text { are recast as } \\
\Lambda_{v \pm}^{-}(s-1, m \pm 1)= \pm \frac{c_{ \pm}^{s m}}{s+1 \pm m} \Lambda_{\nu 3}^{-}(s-1, m \pm 1), \\
\Lambda_{v \pm}^{3}(s, m \pm 1)=\frac{c_{ \pm}^{s m}}{m \pm 1} \Lambda_{v 3}^{3}(s, m \pm 1), \\
\Lambda_{v \pm}^{+}(s+1, m \pm 1)=\mp \frac{c_{ \pm}^{s m}}{s \mp m} \Lambda_{\nu 3}^{+}(s+1, m \pm 1) .
\end{gathered}
$$


The substitution of Eqs. (B20)-(B22) into Eq. (B8) yields

$$
\begin{aligned}
s_{\nu \pm}|s, m, i\rangle= & \pm \frac{c_{ \pm}^{s m}}{s \pm m+1} \Lambda_{\nu 3}^{-}(s-1, m \pm 1)|s, m \pm 1, i\rangle \\
& +\frac{c_{ \pm}^{s m}}{m \pm 1} \Lambda_{\nu 3}^{3}(s, m \pm 1)|s, m \pm 1, i\rangle \\
& \mp \frac{c_{ \pm}^{s m}}{s \mp m} \Lambda_{\nu 3}^{+}(s+1, m \pm 1)|s, m \pm 1, i\rangle
\end{aligned}
$$

The operator $s_{v 3}$ is self-adjoint, i.e., $s_{v 3}=s_{v 3}^{\dagger}$. Taking into account the spin selection rule (B1), the self-adjoint property of the operator $s_{\nu 3}$ is expressed explicitly as

$$
\left\langle s^{\prime}, m, i^{\prime}\left|s_{v 3}\right| s, m, i\right\rangle=\left\langle s, m, i\left|s_{v 3}\right| s^{\prime}, m, i^{\prime}\right\rangle^{*} .
$$

For $s^{\prime}=s$ and $s^{\prime}=s \pm 1$, the conditions (B7) and (B25) require that

$$
\begin{aligned}
& \Lambda_{v 3}^{3}(s, m)=\Lambda_{v 3}^{3}(s, m)^{\dagger}, \\
& \Lambda_{v 3}^{ \pm}(s \pm 1, m)=\Lambda_{v 3}^{\mp}(s, m)^{\dagger},
\end{aligned}
$$

which shows that the operator $\Lambda_{\nu 3}^{3}(s, m)$ is self-adjoint but the operators $\Lambda_{v 3}^{ \pm}(s \pm 1, m)$ are not. The operators $s_{v \pm}$ are adjoint to each other; i.e., $s_{v \mp}=s_{v \pm}^{\dagger}$ according to the definition (238). Taking into account the spin selection rule (B2), the adjoint $s_{\nu \pm}$ of the operator $s_{v \mp}$ is expressed explicitly as

$$
\left\langle s^{\prime}, m \pm 1, i^{\prime}\left|s_{\nu \pm}\right| s, m, i\right\rangle=\left\langle s, m, i\left|s_{\nu \mp}\right| s^{\prime}, m \pm 1, i^{\prime}\right\rangle^{*} .
$$

Taking into account the decomposition (B23) of the operator $s_{v \pm}$ and the condition (B7) for $s^{\prime}=s$, the relation (B28) requires that

$$
\frac{c_{ \pm}^{s m}}{m \pm 1} \Lambda_{\nu 3}^{3}(s, m \pm 1)=\frac{c_{\mp}^{s m \pm 1}}{m} \Lambda_{\nu 3}^{3}(s, m)^{\dagger} .
$$

Using the condition (B26) and the definition (249) of the coefficients $c_{ \pm}^{s m}$, the relation (B29) is recast as

$$
\frac{\Lambda_{v 3}^{3}(s, m \pm 1)}{m \pm 1}=\frac{\Lambda_{v 3}^{3}(s, m)}{m} .
$$

Thus, the operator $\Lambda_{v 3}^{3}(s, m)$ can be recast as

$$
\Lambda_{v 3}^{3}(s, m) \equiv m \Lambda_{v}^{3}(s),
$$

where the operator $\Lambda_{v}^{3}(s)$ is independent of $m$. Taking into account the decomposition (B23) of the operator $s_{v \pm}$ and the condition (B7), the relation (B28) requires that

$$
\frac{c_{ \pm}^{s m}}{s \pm m+1} \Lambda_{\nu 3}^{-}(s-1, m \pm 1)=\frac{c_{\mp}^{s-1 m \pm 1}}{s \pm m} \Lambda_{\nu 3}^{+}(s, m)^{\dagger}
$$

Using the condition (B27) and the definition (249) of the coefficients $c_{ \pm}^{s m}$, the relation (B32) is recast as

$$
\frac{\Lambda_{\nu 3}^{-}(s-1, m \pm 1)}{\sqrt{s^{2}-(m \pm 1)^{2}}}=\frac{\Lambda_{\nu 3}^{-}(s-1, m)}{\sqrt{s^{2}-m^{2}}} .
$$

Thus, the operator $\Lambda_{\nu 3}^{-}(s-1, m)$ can be recast as

$$
\begin{aligned}
\Lambda_{\nu 3}^{-}(s-1, m) & \equiv \sqrt{s^{2}-m^{2}} \Lambda_{\nu}^{-}(s-1) \\
& =\sqrt{(s-m)(s+m)} \Lambda_{\nu}^{-}(s-1),
\end{aligned}
$$

where the operator $\Lambda_{v}^{-}(s-1)$ is independent of $m$. Taking into account the decomposition (B23) of the operator $s_{v \pm}$ and the condition (B7), the relation (B28) requires that

$$
\frac{c_{ \pm}^{s m}}{s \mp m} \Lambda_{\nu 3}^{+}(s+1, m \pm 1)=\frac{c_{\mp}^{s+1 m \pm 1}}{s \mp m+1} \Lambda_{\nu 3}^{-}(s, m)^{\dagger} .
$$

Using the condition (B27) and the definition (249) of the coefficients $c_{ \pm}^{s m}$, the relation (B35) is recast as

$$
\frac{\Lambda_{\nu 3}^{+}(s+1, m \pm 1)}{\sqrt{(s+1)^{2}-(m \pm 1)^{2}}}=\frac{\Lambda_{\nu 3}^{+}(s+1, m)}{\sqrt{(s+1)^{2}-m^{2}}} .
$$

Thus, the operator $\Lambda_{v 3}^{+}(s+1, m)$ can be recast as

$$
\begin{aligned}
\Lambda_{\nu 3}^{+}(s+1, m) & \equiv \sqrt{(s+1)^{2}-m^{2}} \Lambda_{v}^{+}(s+1) \\
& =\sqrt{(s-m+1)(s+m+1)} \Lambda_{v}^{+}(s+1),
\end{aligned}
$$

where the operator $\Lambda_{v}^{+}(s+1)$ is independent of $m$. The substitution of the expressions (B31), (B34) and (B37) in the relations (B26) and (B27) yields

$$
\begin{aligned}
& \Lambda_{v}^{3}(s)=\Lambda_{v}^{3}(s)^{\dagger}, \\
& \Lambda_{v}^{ \pm}(s \pm 1)=\Lambda_{v}^{\mp}(s)^{\dagger} .
\end{aligned}
$$

Using the expressions (B31), (B34), and (B37) and the definition (249), the decompositions (B5) and (B23) are recast, respectively, as

$$
\begin{aligned}
s_{v 3}|s, m, i\rangle= & \sqrt{(s-m)(s+m)} \Lambda_{v}^{-}(s-1)|s, m, i\rangle \\
& +m \Lambda_{v}^{3}(s)|s, m, i\rangle \\
& +\sqrt{(s-m+1)(s+m+1)} \Lambda_{v}^{+}(s+1)|s, m, i\rangle, \\
s_{v \pm}|s, m, i\rangle= & \pm \sqrt{(s \mp m-1)(s \mp m)} \Lambda_{v}^{-}(s-1)|s, m \pm 1, i\rangle \\
& +\sqrt{(s \mp m)(s \pm m+1)} \Lambda_{v}^{3}(s)|s, m \pm 1, i\rangle \\
& \mp \sqrt{(s \pm m+1)(s \pm m+2)} \\
& \times \Lambda_{v}^{+}(s+1)|s, m \pm 1, i\rangle .
\end{aligned}
$$

Proposition 2. For electrons, which are spin- $\frac{1}{2}$ particles, the spectral decomposition of the operators $\Lambda_{v}^{3}(s), \Lambda_{v}^{-}(s) \Lambda_{v}^{-}(s)^{\dagger}$, and $\Lambda_{v}^{+}(s) \Lambda_{v}^{+}(s)^{\dagger}$ reads

$$
\begin{aligned}
& \Lambda_{v}^{3}(s)=\lambda_{s}^{-} \mathcal{P}_{v}^{-}(s)+\lambda_{s}^{+} \mathcal{P}_{v}^{+}(s), \\
& \Lambda_{v}^{-}(s) \Lambda_{v}^{-}(s)^{\dagger}=\lambda_{s}^{-2} \mathcal{P}_{v}^{-}(s), \\
& \Lambda_{v}^{+}(s) \Lambda_{v}^{+}(s)^{\dagger}=\lambda_{s}^{+2} \mathcal{P}_{v}^{+}(s),
\end{aligned}
$$

where $\mathcal{P}_{v}^{ \pm}(s)$ are orthogonal projectors that satisfy the conditions

$$
\begin{array}{ll}
\mathcal{P}_{v}^{ \pm}(s)=\mathcal{P}_{v}^{ \pm}(s)^{\dagger}, & \mathcal{P}_{v}^{\mp}(s) \mathcal{P}_{v}^{ \pm}(s)=0, \\
\mathcal{P}_{v}^{ \pm}(s)^{2}=\mathcal{P}_{v}^{ \pm}(s), & \mathcal{P}_{v}^{-}(s)+\mathcal{P}_{v}^{+}(s)=\mathbb{1}_{d_{s}} .
\end{array}
$$

The eigenvalues $\lambda_{s}^{-}$and $\lambda_{s}^{+}$yield

$$
\begin{aligned}
& \lambda_{s}^{-}=-\frac{1}{2(s+1)}, \\
& \lambda_{s}^{+}=\frac{1}{2 s},
\end{aligned}
$$


and their multiplicities $m_{s}^{-}$and $m_{s}^{+}$are given by

$$
\begin{aligned}
& m_{s}^{-}=\frac{2(s+1)\left(\frac{n}{2}-s\right)}{n\left(\frac{n}{2}+s+1\right)} \frac{n !}{\left(\frac{n}{2}+s\right) !\left(\frac{n}{2}-s\right) !}, \\
& m_{s}^{+}=\frac{2 s}{n} \frac{n !}{\left(\frac{n}{2}+s\right) !\left(\frac{n}{2}-s\right) !} .
\end{aligned}
$$

Proof. For electrons, which are spin- $\frac{1}{2}$ particles, the operator $s_{v 3}^{2}$ is a multiple of the identity operator, i.e.,

$$
s_{v 3}^{2}=\frac{1}{4} \mathbb{1},
$$

which implies that

$$
s_{\nu 3}\left(s_{\nu 3}|s, m, i\rangle\right)=\frac{1}{4}|s, m, i\rangle .
$$

Substituting the expression (B40) twice in the eigenvalue equation (B51), we obtain the following conditions for the operators acting on the Hilbert spaces $\mathcal{H}_{m}^{s \pm 2}, \mathcal{H}_{m}^{s \pm 1}$, and $\mathcal{H}_{m}^{s}$, respectively,

$$
\begin{aligned}
& \Lambda_{v}^{ \pm}(s \pm 2) \Lambda_{v}^{ \pm}(s \pm 1)=0, \\
& \Lambda_{v}^{ \pm}(s \pm 1) \Lambda_{v}^{3}(s)+\Lambda_{v}^{3}(s \pm 1) \Lambda_{v}^{ \pm}(s \pm 1)=0, \\
& \left(s^{2}-m^{2}\right) \Lambda_{v}^{+}(s) \Lambda_{v}^{-}(s-1)+m^{2} \Lambda_{v}^{3}(s)^{2} \\
& +\left[(s+1)^{2}-m^{2}\right] \Lambda_{v}^{-}(s) \Lambda_{v}^{+}(s+1)=\frac{1}{4} \mathbb{1}_{d_{s}} .
\end{aligned}
$$

Using the property (B39), the relation (B54) is recast as

$$
\begin{aligned}
& \left(s^{2}-m^{2}\right) \Lambda_{v}^{+}(s) \Lambda_{v}^{+}(s)^{\dagger}+m^{2} \Lambda_{v}^{3}(s)^{2} \\
& +\left[(s+1)^{2}-m^{2}\right] \Lambda_{v}^{-}(s) \Lambda_{v}^{-}(s)^{\dagger}=\frac{1}{4} \mathbb{1}_{d_{s}} .
\end{aligned}
$$

Using the relation (B39) and the condition (B52), the selfadjoint operators $\Lambda_{v}^{+}(s) \Lambda_{v}^{+}(s)^{\dagger}$ and $\Lambda_{v}^{-}(s) \Lambda_{v}^{-}(s)^{\dagger}$ satisfy the commutation relation

$$
\begin{aligned}
& {\left[\Lambda_{v}^{+}(s) \Lambda_{v}^{+}(s)^{\dagger}, \Lambda_{v}^{-}(s) \Lambda_{v}^{-}(s)^{\dagger}\right]} \\
& =\Lambda_{v}^{+}(s)\left[\Lambda_{v}^{-}(s-1) \Lambda_{v}^{-}(s)\right] \Lambda_{v}^{+}(s+1) \\
& \quad-\Lambda_{v}^{-}(s)\left[\Lambda_{v}^{+}(s+1) \Lambda_{v}^{+}(s)\right] \Lambda_{v}^{-}(s-1)=0 .
\end{aligned}
$$

Using the relation (B39) and the condition (B53), the selfadjoint operators $\Lambda_{v}^{3}(s)$ and $\Lambda_{v}^{+}(s) \Lambda_{v}^{+}(s)^{\dagger}$ satisfy the commutation relation

$$
\begin{aligned}
& {\left[\Lambda_{v}^{3}(s), \Lambda_{v}^{+}(s) \Lambda_{v}^{+}(s)^{\dagger}\right]} \\
& =\Lambda_{v}^{3}(s) \Lambda_{v}^{+}(s) \Lambda_{v}^{-}(s-1)-\Lambda_{v}^{+}(s) \Lambda_{v}^{-}(s-1) \Lambda_{v}^{3}(s) \\
& =-\Lambda_{v}^{+}(s)\left[\Lambda_{v}^{3}(s-1) \Lambda_{v}^{-}(s-1)+\Lambda_{v}^{-}(s-1) \Lambda_{v}^{3}(s)\right] \\
& =0 .
\end{aligned}
$$

Using the relation (B39) and the condition (B53), the selfadjoint operators $\Lambda_{v}^{3}(s)$ and $\Lambda_{v}^{-}(s) \Lambda_{v}^{-}(s)^{\dagger}$ satisfy the commutation relation

$$
\begin{aligned}
& {\left[\Lambda_{v}^{3}(s), \Lambda_{v}^{-}(s) \Lambda_{v}^{-}(s)^{\dagger}\right]} \\
& =\Lambda_{v}^{3}(s) \Lambda_{v}^{-}(s) \Lambda_{v}^{+}(s+1)-\Lambda_{v}^{-}(s) \Lambda_{v}^{+}(s+1) \Lambda_{v}^{3}(s) \\
& =-\Lambda_{v}^{-}(s)\left[\Lambda_{v}^{3}(s+1) \Lambda_{v}^{+}(s+1)+\Lambda_{v}^{+}(s+1) \Lambda_{v}^{3}(s)\right] \\
& =0 .
\end{aligned}
$$

The relations (B56), (B57), and (B58) imply that the selfadjoint operators $\Lambda_{v}^{+}(s) \Lambda_{v}^{+}(s)^{\dagger}, \Lambda_{v}^{-}(s) \Lambda_{v}^{-}(s)^{\dagger}$, and $\Lambda_{v}^{3}(s)$ commute.

For electrons, which are spin- $\frac{1}{2}$ particles, the operators $s_{v \pm}^{2}$ vanish; i.e.,

$$
s_{v \pm}^{2}=0
$$

which implies that

$$
s_{\nu \pm}\left(s_{\nu \pm}|s, m, i\rangle\right)=0 .
$$

Substituting the expression (B41) twice in the eigenvalue equation (B60), we obtain the following conditions for the operators acting on the Hilbert spaces $\mathcal{H}_{A m \pm 2}^{s \pm 2}, \mathcal{H}_{A m \pm 1}^{s \pm 1}$, and $\mathcal{H}_{m}^{s}$, respectively:

$$
\begin{aligned}
& \Lambda_{v}^{ \pm}(s \pm 2) \Lambda_{v}^{ \pm}(s \pm 1)=0, \\
& \Lambda_{v}^{ \pm}(s \pm 1) \Lambda_{v}^{3}(s)+\Lambda_{v}^{3}(s \pm 1) \Lambda_{v}^{ \pm}(s \pm 1)=0, \\
& \Lambda_{v}^{+}(s) \Lambda_{v}^{-}(s-1)-\Lambda_{v}^{3}(s)^{2}+\Lambda_{v}^{-}(s) \Lambda_{v}^{+}(s+1)=0 .
\end{aligned}
$$

Using the property (B39), the relation (B63) is recast as

$$
\Lambda_{v}^{+}(s) \Lambda_{v}^{+}(s)^{\dagger}-\Lambda_{v}^{3}(s)^{2}+\Lambda_{v}^{-}(s) \Lambda_{v}^{-}(s)^{\dagger}=0 .
$$

Factorizing $m^{2}$ in the condition (B55) and substituting the relation (B64) yields

$$
s^{2} \Lambda_{v}^{+}(s) \Lambda_{v}^{+}(s)^{\dagger}+(s+1)^{2} \Lambda_{v}^{-}(s) \Lambda_{v}^{-}(s)^{\dagger}=\frac{1}{4} \mathbb{1}_{d_{s}} .
$$

Substituting the condition (B64) into the condition (B55), we express respectively the self-adjoint operators $\Lambda_{v}^{-}(s) \Lambda_{v}^{-}(s)^{\dagger}$ and $\Lambda_{v}^{+}(s) \Lambda_{v}^{+}(s)^{\dagger}$ in terms of the self-adjoint operator $\Lambda_{v}^{3}(s)$ as

$$
\begin{aligned}
& \Lambda_{v}^{-}(s) \Lambda_{v}^{-}(s)^{\dagger}=\frac{1}{2 s+1}\left[\frac{1}{4} \mathbb{1}_{d_{s}}-s^{2} \Lambda_{v}^{3}(s)^{2}\right], \\
& \Lambda_{v}^{+}(s) \Lambda_{v}^{+}(s)^{\dagger}=\frac{1}{2 s+1}\left[(s+1)^{2} \Lambda_{v}^{3}(s)^{2}-\frac{1}{4} \mathbb{1}_{d_{s}}\right] .
\end{aligned}
$$

Using the definition (238), the commutation relation

$$
\left[s_{v j}, s_{v^{\prime} k}\right]=i \delta_{v v^{\prime}} \varepsilon_{i j k} \delta^{i \ell} s_{\nu \ell},
$$

and the property

$$
s_{v 1}^{2}=s_{v 2}^{2}=\frac{1}{4} \mathbb{1}
$$

we obtain the identity

$$
s_{\nu \pm} s_{\nu \mp}=\frac{1}{2} \mathbb{1} \pm s_{v 3}
$$

which implies that

$$
s_{\nu \pm}\left(s_{\nu \mp}|s, m, i\rangle\right)=\frac{1}{2}|s, m, i\rangle \pm s_{\nu 3}|s, m, i\rangle .
$$

Substituting the expression (B41) twice in the eigenvalue equation (B71), we obtain the following conditions for the operators acting on the Hilbert spaces $\mathcal{H}_{m}^{s-2}, \mathcal{H}_{m}^{s+2}, \mathcal{H}_{m}^{s-1}$, $\mathcal{H}_{m}^{s+1}$, and $\mathcal{H}_{m}^{s}$, respectively,

$$
\begin{aligned}
& \Lambda_{v}^{-}(s-2) \Lambda_{v}^{-}(s-1)=0, \\
& \Lambda_{v}^{+}(s+2) \Lambda_{v}^{+}(s+1)=0,
\end{aligned}
$$




$$
\begin{aligned}
& (s \mp m+1) \Lambda_{v}^{-}(s-1) \Lambda_{v}^{3}(s) \\
& -(s \pm m-1) \Lambda_{v}^{3}(s-1) \Lambda_{v}^{-}(s-1)=\Lambda_{v}^{-}(s-1), \\
& (s \mp m+2) \Lambda_{v}^{3}(s+1) \Lambda_{v}^{+}(s+1) \\
& -(s \pm m) \Lambda_{v}^{+}(s+1) \Lambda_{v}^{3}(s)=\Lambda_{v}^{+}(s+1), \\
& (s \pm m-1)(s \pm m) \Lambda_{v}^{+}(s) \Lambda_{v}^{-}(s-1) \\
& +(s \mp m+1)(s \pm m) \Lambda_{v}^{3}(s)^{2} \\
& +(s \mp m+1)(s \mp m+2) \Lambda_{v}^{-}(s) \Lambda_{v}^{+}(s+1) \\
& \quad=\frac{1}{2} \mathbb{1}_{d_{s}} \pm m \Lambda_{v}^{3}(s) .
\end{aligned}
$$

Factorizing $m$ in the condition (B74) and substituting the relation (B62) with a negative sign yields

$$
\begin{aligned}
& (s+1) \Lambda_{v}^{-}(s-1) \Lambda_{v}^{3}(s)-(s-1) \Lambda_{v}^{3}(s-1) \Lambda_{v}^{-}(s-1) \\
& =\Lambda_{v}^{-}(s-1) .
\end{aligned}
$$

Factorizing $m$ in the condition (B75) and substituting the relation (B62) with a positive sign yields

$$
\begin{aligned}
& (s+2) \Lambda_{v}^{3}(s+1) \Lambda_{v}^{+}(s+1)-s \Lambda_{v}^{+}(s+1) \Lambda_{v}^{3}(s) \\
& =\Lambda_{v}^{+}(s+1) .
\end{aligned}
$$

Using the property (B39), the relation (B76) is recast as

$$
\begin{aligned}
& {\left[s(s-1) \pm m(2 s-1)+m^{2}\right] \Lambda_{v}^{+}(s) \Lambda_{v}^{+}(s)^{\dagger}} \\
& +\left[s(s+1) \pm m-m^{2}\right] \Lambda_{v}^{3}(s)^{2} \\
& +\left[(s+1)(s+2) \mp m(2 s+3)+m^{2}\right] \Lambda_{v}^{-}(s) \Lambda_{v}^{-}(s)^{\dagger} \\
& \quad=\frac{1}{2} \mathbb{1}_{d_{s}} \pm m \Lambda_{v}^{3}(s) .
\end{aligned}
$$

Factorizing $m^{2}$ in the condition (B79) and substituting the relation (B64) yields

$$
\begin{aligned}
& {[s(s-1) \pm m(2 s-1)] \Lambda_{v}^{+}(s) \Lambda_{v}^{+}(s)^{\dagger}+[s(s+1) \pm m] \Lambda_{v}^{3}(s)^{2}} \\
& +[(s+1)(s+2) \mp m(2 s+3)] \Lambda_{v}^{-}(s) \Lambda_{v}^{-}(s)^{\dagger} \\
& \quad=\frac{1}{2} \mathbb{1}_{d_{s}} \pm m \Lambda_{v}^{3}(s) .
\end{aligned}
$$

The condition (B80) has to hold for any value of $m$. Thus, the terms multiplying $m$ in the relation (B80) and the remaining terms have to vanish separately, which yields two conditions:

$$
\begin{aligned}
& (2 s-1) \Lambda_{v}^{+}(s) \Lambda_{v}^{+}(s)^{\dagger}+\Lambda_{v}^{3}(s)^{2} \\
& -(2 s+3) \Lambda_{v}^{-}(s) \Lambda_{v}^{-}(s)^{\dagger}=\Lambda_{v}^{3}(s), \\
& s(s-1) \Lambda_{v}^{+}(s) \Lambda_{v}^{+}(s)^{\dagger}+s(s+1) \Lambda_{v}^{3}(s)^{2} \\
& +(s+1)(s+2) \Lambda_{v}^{-}(s) \Lambda_{v}^{-}(s)^{\dagger}=\frac{1}{2} \mathbb{1}_{d_{s}} .
\end{aligned}
$$

The substitution of the relations (B67) and (B66) into the relation (B81) yields

$$
2 s(s+1) \Lambda_{v}^{3}(s)^{2}-\frac{1}{2} \mathbb{1}_{d_{s}}=\Lambda_{v}^{3}(s) .
$$

Subtracting the condition (B62) with a negative sign from the condition (B77) yields

$$
s\left[\Lambda_{v}^{-}(s-1) \Lambda_{v}^{3}(s)-\Lambda_{v}^{3}(s-1) \Lambda_{v}^{-}(s-1)\right]=\Lambda_{v}^{-}(s-1) .
$$

(B84)
According to the condition (B62) with a negative sign, the second term on the left-hand side of the condition (B84) is the opposite of the first. Thus, under the transformation $s \rightarrow$ $s+1$, the condition (B84) yields the eigenvalue equation

$$
\Lambda_{v}^{3}(s) \Lambda_{v}^{-}(s)=\lambda_{s}^{-} \Lambda_{v}^{-}(s),
$$

where

$$
\lambda_{s}^{-}=-\frac{1}{2(s+1)} .
$$

Subtracting the condition (B62) with a positive sign from the condition (B78) yields

$$
\begin{aligned}
& (s+1)\left[\Lambda_{v}^{3}(s+1) \Lambda_{v}^{+}(s+1)-\Lambda_{v}^{+}(s+1) \Lambda_{v}^{3}(s)\right] \\
& \quad=\Lambda_{v}^{+}(s+1) .
\end{aligned}
$$

According to the condition (B62) with a positive sign, the second term on the left-hand side of the condition (B87) is the opposite of the first. Thus, under the transformation $s \rightarrow$ $s-1$, the condition (B87) yields the eigenvalue equation

$$
\Lambda_{v}^{3}(s) \Lambda_{v}^{+}(s)=\lambda_{s}^{+} \Lambda_{v}^{+}(s),
$$

where

$$
\lambda_{s}^{+}=\frac{1}{2 s}
$$

The eigenvalue equations (B85) and (B88) imply that the selfadjoint operator $\Lambda_{v}^{3}(s)$ is given by

$$
\Lambda_{v}^{3}(s)=\lambda_{s}^{-} \mathcal{P}_{v}^{-}(s)+\lambda_{s}^{+} \mathcal{P}_{v}^{+}(s),
$$

where the projectors $\mathcal{P}_{v}^{ \pm}(s)$ satisfy the following conditions:

$$
\begin{aligned}
\mathcal{P}_{v}^{ \pm}(s) & =\mathcal{P}_{v}^{ \pm}(s)^{\dagger}, \quad \mathcal{P}_{v}^{\mp}(s) \mathcal{P}_{v}^{ \pm}(s)=0, \\
\mathcal{P}_{v}^{ \pm}(s)^{2} & =\mathcal{P}_{v}^{ \pm}(s), \quad \mathcal{P}_{v}^{-}(s)+\mathcal{P}_{v}^{+}(s)=\mathbb{1}_{d_{s}} .
\end{aligned}
$$

The relations (B98) and (B99), the property (B39) and the conditions (B72) and (B73) imply that

$$
\begin{aligned}
\mathcal{P}_{v}^{+}(s) \Lambda_{v}^{-}(s) & =\lambda_{s}^{+2} \Lambda_{v}^{+}(s) \Lambda_{v}^{+}(s)^{\dagger} \Lambda_{v}^{-}(s) \\
& =\lambda_{s}^{+2} \Lambda_{v}^{+}(s)\left[\Lambda_{v}^{-}(s-1) \Lambda_{v}^{-}(s)\right]=0, \\
\mathcal{P}_{v}^{-}(s) \Lambda_{v}^{+}(s) & =\lambda_{s}^{-2} \Lambda_{v}^{-}(s) \Lambda_{v}^{-}(s)^{\dagger} \Lambda_{v}^{+}(s) \\
& =\lambda_{s}^{-2} \Lambda_{v}^{-}(s)\left[\Lambda_{v}^{+}(s+1) \Lambda_{v}^{+}(s)\right]=0 .
\end{aligned}
$$

The relations (B90), (B92), and (B93) imply that

$$
\begin{aligned}
& \mathcal{P}_{v}^{-}(s) \Lambda_{v}^{-}(s)=\left(\lambda_{s}^{-}\right)^{-1}\left[\Lambda_{v}^{3}(s)-\lambda_{s}^{+} \mathcal{P}_{v}^{+}(s)\right] \Lambda_{v}^{-}(s)=\Lambda_{v}^{-}(s), \\
& \mathcal{P}_{v}^{+}(s) \Lambda_{v}^{+}(s)=\left(\lambda_{s}^{+}\right)^{-1}\left[\Lambda_{v}^{3}(s)-\lambda_{s}^{-} \mathcal{P}_{v}^{-}(s)\right] \Lambda_{v}^{+}(s)=\Lambda_{v}^{+}(s) .
\end{aligned}
$$

Using the definitions (B86) and (B89) of the eigenvalues $\lambda_{ \pm}(s)$ and the properties (B91) of the projectors $\mathcal{P}_{v}^{ \pm}(s)$ and substituting the expression (B90) for the operator $\Lambda_{v}^{3}(s)$ into 
the relations (B66) and (B67) yields

$$
\begin{aligned}
\Lambda_{v}^{-}(s) \Lambda_{v}^{-}(s)^{\dagger}= & \frac{1}{2 s+1}\left\{\frac{1}{4}\left[\mathcal{P}_{v}^{-}(s)+\mathcal{P}_{v}^{+}(s)\right]\right. \\
& \left.-\frac{s^{2}}{4(s+1)^{2}} \mathcal{P}_{v}^{-}(s)-\frac{s^{2}}{4 s^{2}} \mathcal{P}_{v}^{+}(s)\right\}, \quad \text { (B96) } \\
\Lambda_{v}^{+}(s) \Lambda_{v}^{+}(s)^{\dagger}= & \frac{1}{2 s+1}\left\{\frac{(s+1)^{2}}{4(s+1)^{2}} \mathcal{P}_{v}^{-}(s)+\frac{(s+1)^{2}}{4 s^{2}} \mathcal{P}_{v}^{+}(s)\right. \\
& \left.-\frac{1}{4}\left[\mathcal{P}_{v}^{-}(s)+\mathcal{P}_{v}^{+}(s)\right]\right\} .
\end{aligned}
$$

Using the definitions (B86) and (B89), the relations (B96) and (B97) reduce, respectively, to

$$
\begin{aligned}
& \Lambda_{v}^{-}(s) \Lambda_{v}^{-}(s)^{\dagger}=\lambda_{s}^{-2} \mathcal{P}_{v}^{-}(s), \\
& \Lambda_{v}^{+}(s) \Lambda_{v}^{+}(s)^{\dagger}=\lambda_{s}^{+2} \mathcal{P}_{v}^{+}(s) .
\end{aligned}
$$

Now we determine the multiplicities of the eigenvalues $\lambda_{s}^{-}$and $\lambda_{s}^{+}$. The definition (242) and the eigenvalue equations (247) and (B3) imply that

$$
\sum_{\nu=1}^{n} \Lambda_{v}^{3}(s)=\mathbb{1}_{d_{s}} .
$$

The trace of the relation $(\mathrm{B} 100)$ reads

$$
\operatorname{Tr}\left[\sum_{\nu=1}^{n} \Lambda_{v}^{3}(s)\right]=n \operatorname{Tr}\left[\Lambda_{v}^{3}(s)\right]=d_{s},
$$

which implies that

$$
\operatorname{Tr}\left[\Lambda_{v}^{3}(s)\right]=\frac{d_{s}}{n} .
$$

The multiplicities $m_{s}^{-}$and $m_{s}^{+}$of the eigenvalues $\lambda_{s}^{-}$and $\lambda_{s}^{+}$ of the operator $\Lambda_{v}^{3}(s)$ are defined as

$$
m_{s}^{-}=(1-\alpha) d_{s}, \quad m_{s}^{+}=\alpha d_{s},
$$

where $0 \leqslant \alpha \leqslant 1$ and

$$
m_{s}^{-}+m_{s}^{+}=d_{s} .
$$

Taking into account the spectral decomposition (B90) and the expressions (B86) and (B86) of the eigenvalues $\lambda_{s}^{-}$and $\lambda_{s}^{+}$, the relation (B102) is recast as

$$
(1-\alpha) \lambda_{s}^{-} d_{s}+\alpha \lambda_{s}^{+} d_{s}=\frac{d_{s}}{n},
$$

which implies that the factor $\alpha$ yields

$$
\alpha=\frac{s(n+2 s+2)}{n(2 s+1)} .
$$

For $s=0$, the parameter $\alpha=0$ and for $s=n / 2$, we have $\alpha=1$. Taking into account the expression (30) of the dimension $d_{s}$ and the expression (B106) of $\alpha$, the multiplicities (B103) of the eigenvalues $\lambda_{s}^{-}$and $\lambda_{s}^{+}$are expressed explicitly as

$$
\begin{aligned}
& m_{s}^{-}=\frac{2(s+1)\left(\frac{n}{2}-s\right)}{n\left(\frac{n}{2}+s+1\right)} \frac{n !}{\left(\frac{n}{2}+s\right) !\left(\frac{n}{2}-s\right) !}, \\
& m_{s}^{+}=\frac{2 s}{n} \frac{n !}{\left(\frac{n}{2}+s\right) !\left(\frac{n}{2}-s\right) !}
\end{aligned}
$$

which implies that

$$
m_{s}^{+}=m_{s-1}^{-}
$$

Proposition 3. The operator $\Lambda_{v}^{+}(s)$ and the adjoint operator $\Lambda_{v}^{-}(s-1)$ are expressed as

$$
\begin{aligned}
& \Lambda_{v}^{+}(s)=\lambda_{s}^{+} \mathcal{P}_{v}^{+}(s) X_{v}(s) \mathcal{P}_{v}^{-}(s-1), \\
& \Lambda_{v}^{-}(s-1)=\lambda_{s}^{+} \mathcal{P}_{v}^{-}(s-1) X_{v}(s)^{\dagger} \mathcal{P}_{v}^{+}(s),
\end{aligned}
$$

and satisfy the conditions

$$
\begin{aligned}
& \mathcal{P}_{v}^{+}(s) X_{v}(s) \mathcal{P}_{v}^{-}(s-1) X_{v}(s)^{\dagger} \mathcal{P}_{\nu}^{+}(s)=\mathcal{P}_{v}^{+}(s), \\
& \mathcal{P}_{v}^{-}(s-1) X_{v}(s)^{\dagger} \mathcal{P}_{v}^{+}(s) X_{v}(s) \mathcal{P}_{v}^{-}(s-1)=\mathcal{P}_{v}^{-}(s-1) .
\end{aligned}
$$

Taking into account the definition (B42) and the property (B45), the definitions (B109) and (B110) and the relations (B111) and (B112) satisfy the conditions (B61), (B62), (B64), (B65), (B77), and (B78).

Proof. The expression (B109), the condition (B39), and the properties (B45) imply that

$$
\Lambda_{v}^{+}(s) \Lambda_{v}^{+}(s)^{\dagger}=\lambda_{s}^{+2} \mathcal{P}_{v}^{+}(s) X_{v}(s) \mathcal{P}_{v}^{-}(s-1) X_{v}(s)^{\dagger} \mathcal{P}_{v}^{+}(s)
$$

The relations (B99) and (B113) imply that

$$
\mathcal{P}_{v}^{+}(s) X_{v}(s) \mathcal{P}_{v}^{-}(s-1) X_{v}(s)^{\dagger} \mathcal{P}_{v}^{+}(s)=\mathcal{P}_{v}^{+}(s) .
$$

The expression (B110), the condition (B39), and the properties (B45) imply that

$$
\begin{aligned}
& \Lambda_{v}^{-}(s) \Lambda_{v}^{-}(s)^{\dagger} \\
& \quad=\lambda_{s}^{-2} \mathcal{P}_{v}^{-}(s) X_{v}(s+1)^{\dagger} \mathcal{P}_{v}^{+}(s+1) X_{v}(s+1) \mathcal{P}_{v}^{-}(s) .
\end{aligned}
$$

The relations (B98) and (B115) imply that

$$
\mathcal{P}_{v}^{-}(s) X_{v}(s+1)^{\dagger} \mathcal{P}_{v}^{+}(s+1) X_{v}(s+1) \mathcal{P}_{\nu}^{-}(s)=\mathcal{P}_{v}^{-}(s),
$$

which requires, in turn, that

$$
\mathcal{P}_{v}^{-}(s-1) X_{v}(s)^{\dagger} \mathcal{P}_{v}^{+}(s) X_{v}(s) \mathcal{P}_{v}^{-}(s-1)=\mathcal{P}_{v}^{-}(s-1) .
$$

Proposition 4. For the electron $v=1$, the operators $\Lambda_{1}^{3}(s)$, $\Lambda_{1}^{+}(s+1)$, and $\Lambda_{1}^{-}(s-1)$ are represented by the block matrices

$$
\begin{aligned}
& \Lambda_{1}^{3}(s)=\lambda_{s}^{-}\left(\begin{array}{cc}
\mathbb{1}_{m_{s}^{-}} & 0 \\
0 & 0
\end{array}\right)+\lambda_{s}^{+}\left(\begin{array}{cc}
0 & 0 \\
0 & \mathbb{1}_{m_{s}^{+}}
\end{array}\right), \\
& \Lambda_{1}^{+}(s+1)=\lambda_{s+1}^{+}\left(\begin{array}{cc}
0 & 0 \\
\mathbb{1}_{m_{s}^{-}} & 0
\end{array}\right), \\
& \Lambda_{1}^{-}(s-1)=\lambda_{s}^{+}\left(\begin{array}{cc}
0 & \mathbb{1}_{m_{s}^{+}} \\
0 & 0
\end{array}\right) .
\end{aligned}
$$




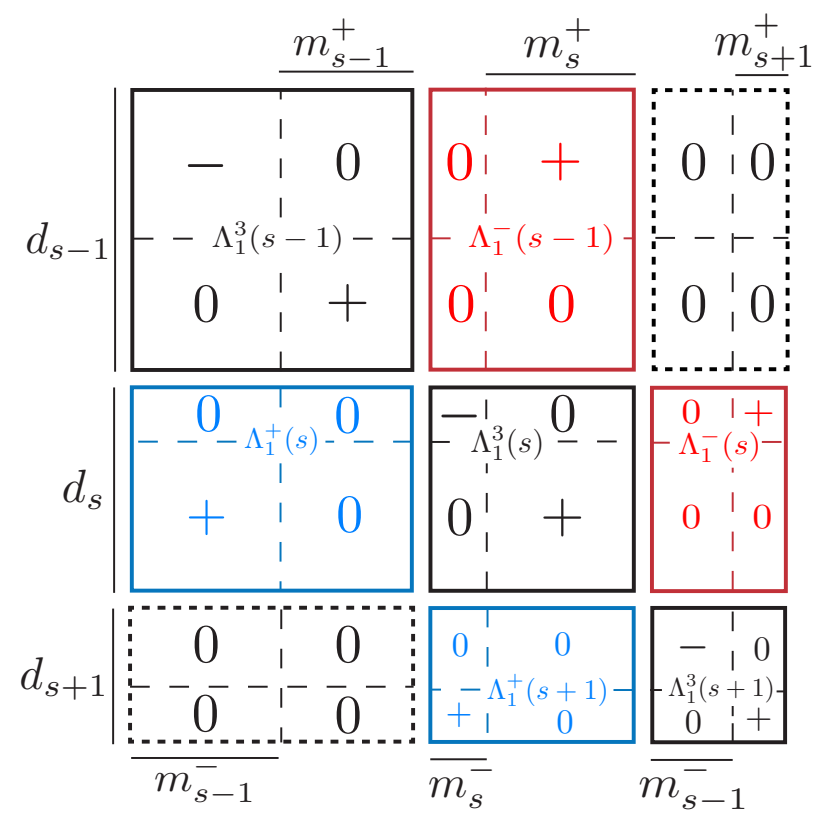

FIG. 8. Tridiagonal matrix representation of the spin operator associated with the electron $v=1$. The blocks are proportional to the operators $\Lambda_{1}^{3}(s), \Lambda_{1}^{3}(s \pm 1), \Lambda_{1}^{ \pm}(s)$, and $\Lambda_{1}^{ \pm}(s \pm 1)$. The " - " sign is associated with a negative eigenvalue and the " + " sign is associated with a positive eigenvalue.

The structure of the tridiagonal matrix representation of the operator $s_{31}$ expressed in terms of blocks that are proportional to the operators $\Lambda_{1}^{3}(s), \Lambda_{1}^{3}(s \pm 1), \Lambda_{1}^{ \pm}(s)$, and $\Lambda_{1}^{ \pm}(s \pm 1)$ is illustrated in Fig. 8 .

Proof. For the electron $v=1$, the orthonormal basis vectors associated with the spin quantum number $s$ can be chosen such that the projectors $\mathcal{P}_{1}^{-}$and $\mathcal{P}_{1}^{+}$are represented by the block matrices

$$
\mathcal{P}_{1}^{-}(s)=\left(\begin{array}{cc}
\mathbb{1}_{m_{s}^{-}} & 0 \\
0 & 0
\end{array}\right) \quad \text { and } \quad \mathcal{P}_{1}^{+}(s)=\left(\begin{array}{cc}
0 & 0 \\
0 & \mathbb{1}_{m_{s}^{+}}
\end{array}\right)
$$

and the operator $X_{1}(s)$ is represented by the block matrix

$$
X_{1}(s)=\left(\begin{array}{ll}
X_{1}^{--}(s) & X_{1}^{-+}(s) \\
X_{1}^{+-}(s) & X_{1}^{++}(s)
\end{array}\right) .
$$

The definition (B90) and the representations (B121) imply that

$$
\Lambda_{1}^{3}(s)=\lambda_{s}^{-}\left(\begin{array}{cc}
\mathbb{1}_{m_{s}^{-}} & 0 \\
0 & 0
\end{array}\right)+\lambda_{s}^{+}\left(\begin{array}{cc}
0 & 0 \\
0 & \mathbb{1}_{m_{s}^{+}}
\end{array}\right) .
$$

The definitions (B109) and (B110) and the representations (B121) and (B122) imply that the operator $\Lambda_{1}^{+}(s)$ is represented as

$$
\Lambda_{1}^{+}(s)=\lambda_{s}^{+}\left(\begin{array}{cc}
0 & 0 \\
X_{1}^{+-}(s) & 0
\end{array}\right)
$$

and the adjoint operator $\Lambda_{1}^{-}(s-1)$ is represented as

$$
\Lambda_{1}^{-}(s-1)=\lambda_{s}^{+}\left(\begin{array}{cc}
0 & X_{1}^{+-}(s)^{\dagger} \\
0 & 0
\end{array}\right),
$$

which, in turn, implies that

$$
\Lambda_{1}^{+}(s+1)=\lambda_{s+1}^{+}\left(\begin{array}{cc}
0 & 0 \\
X_{1}^{+-}(s+1) & 0
\end{array}\right)
$$

Taking into account the representations (B121) and (B122), the condition (B114) requires that

$$
X_{1}^{+-}(s) X_{1}^{+-}(s)^{\dagger}=\mathbb{1}_{m_{s}^{+}} .
$$

Taking into account the representations (B121) and (B122), the condition (B115) requires that

$$
X_{1}^{+-}(s+1)^{\dagger} X_{1}^{+-}(s+1)=\mathbb{1}_{m_{s}^{-}} .
$$

According to the property (B108), the condition (B128) is recast as

$$
X_{1}^{+-}(s)^{\dagger} X_{1}^{+-}(s)=\mathbb{1}_{m_{s-1}^{-}}=\mathbb{1}_{m_{s}^{+}} .
$$

In order to satisfy the conditions (B127) and (B129), the operator $X_{1}^{+-}(s)$ can be chosen such that

$$
X_{1}^{+-}(s)=\mathbb{1}_{m_{s}^{+}}
$$

The expression (B130) implies that the representation (B125) of the operator $\Lambda_{1}^{-}(s-1)$ is recast as

$$
\Lambda_{1}^{-}(s-1)=\lambda_{s}^{+}\left(\begin{array}{cc}
0 & \mathbb{1}_{m_{s}^{+}} \\
0 & 0
\end{array}\right)
$$

The conditions (B130) and (B108) imply that

$$
X_{1}^{+-}(s+1)=\mathbb{1}_{m_{s+1}^{+}}=\mathbb{1}_{m_{s}^{-}} .
$$

The expression (B132) implies that the representation (B126) of the operator $\Lambda_{1}^{+}(s+1)$ is recast as

$$
\Lambda_{1}^{+}(s+1)=\lambda_{s+1}^{+}\left(\begin{array}{cc}
0 & 0 \\
\mathbb{1}_{m_{s}^{-}} & 0
\end{array}\right)
$$

Proposition 5. For the electron $v=1$, the reduced matrix elements of the operators $s_{31}$ and $s_{ \pm 1}$ read

$$
\begin{aligned}
& \sum_{i^{\prime}=1}^{d_{s^{\prime}}} \sum_{i=1}^{d_{s}}\left\langle s^{\prime}, m^{\prime}, i^{\prime}\left|s_{13}\right| s, m, i\right\rangle \\
&=+\lambda_{s}^{+} m_{s}^{+} \sqrt{(s-m)(s+m)} \delta_{s^{\prime} s-1} \delta_{m^{\prime} m}+\frac{d_{s}}{n} m \delta_{s^{\prime} s} \delta_{m^{\prime} m} \\
&+\lambda_{s+1}^{+} m_{s}^{-} \sqrt{(s-m+1)(s+m+1)} \delta_{s^{\prime} s+1} \delta_{m^{\prime} m}, \quad \text { B } \\
& \sum_{i^{\prime}=1}^{d_{s^{\prime}}} \sum_{i=1}^{d_{s}}\left\langle s^{\prime}, m^{\prime}, i^{\prime}\left|s_{1 \pm}\right| s, m, i\right\rangle \\
&= \pm \lambda_{s}^{+} m_{s}^{+} \sqrt{(s \mp m-1)(s \mp m)} \delta_{s^{\prime} s-1} \delta_{m^{\prime} m \pm 1} \\
& \quad+\frac{d_{s}}{n} \sqrt{(s \mp m)(s \pm m+1)} \delta_{s^{\prime} s} \delta_{m^{\prime} m \pm 1} \\
& \mp \lambda_{s+1}^{+} m_{s}^{-} \sqrt{(s \pm m+1)(s \pm m+2)} \delta_{s^{\prime} s+1} \delta_{m^{\prime} m \pm 1} . \quad \text { (B }
\end{aligned}
$$


Proof. For the electron $v=1$, the eigenvalue equation (B3) yields the reduced matrix element of the operator $s_{31}$; i.e.,

$$
\begin{aligned}
\sum_{i^{\prime}=1}^{d_{s^{\prime}}} \sum_{i=1}^{d_{s}}\left\langle s^{\prime}, m^{\prime}, i^{\prime}\left|s_{13}\right| s, m, i\right\rangle= & +\sqrt{(s-m)(s+m)} \sum_{i^{\prime}=1}^{d_{s^{\prime}}} \sum_{i=1}^{d_{s}}\left\langle s^{\prime}, m^{\prime}, i^{\prime}\left|\Lambda_{1}^{3}(s-1)\right| s, m, i\right\rangle+m \sum_{i^{\prime}=1}^{d_{s^{\prime}}} \sum_{i=1}^{d_{s}}\left\langle s^{\prime}, m^{\prime}, i^{\prime}\left|\Lambda_{1}^{3}(s)\right| s, m, i\right\rangle \\
& +\sqrt{(s-m+1)(s+m+1)} \sum_{i^{\prime}=1}^{d_{s^{\prime}}} \sum_{i=1}^{d_{s}}\left\langle s^{\prime}, m^{\prime}, i^{\prime}\left|\Lambda_{1}^{3}(s+1)\right| s, m, i\right\rangle .
\end{aligned}
$$

Similarly, the eigenvalue equation (B4) yields the matrix element associated with the operators $s_{1 \pm}$; i.e.,

$$
\begin{aligned}
& \sum_{i^{\prime}=1}^{d_{s^{\prime}}} \sum_{i=1}^{d_{s}}\left\langle s^{\prime}, m^{\prime}, i^{\prime}\left|s_{1 \pm}\right| s, m, i\right\rangle= \pm \sqrt{(s \mp m-1)(s \mp m)} \sum_{i^{\prime}=1}^{d_{s^{\prime}}} \sum_{i=1}^{d_{s}}\left\langle s^{\prime}, m^{\prime}, i^{\prime}\left|\Lambda_{1}^{3}(s-1)\right| s, m \pm 1, i\right\rangle \\
&+\sqrt{(s \mp m)(s \pm m+1)} \sum_{i^{\prime}=1}^{d_{s^{\prime}}} \sum_{i=1}^{d_{s}}\left\langle s^{\prime}, m^{\prime}, i^{\prime}\left|\Lambda_{1}^{3}(s)\right| s, m \pm 1, i\right\rangle \\
& \mp \sqrt{(s \pm m+1)(s \pm m+2)} \sum_{i^{\prime}=1}^{d_{s^{\prime}}} \sum_{i=1}^{d_{s}}\left\langle s^{\prime}, m^{\prime}, i^{\prime}\left|\Lambda_{1}^{3}(s+1)\right| s, m \pm 1, i\right\rangle .
\end{aligned}
$$

The representation (B118) and the expressions (30), (B86), (B89), and (B107) imply that, $\forall s, m$,

$$
\sum_{i^{\prime}=1}^{d_{s^{\prime}}} \sum_{i=1}^{d_{s}}\left\langle s^{\prime}, m^{\prime}, i^{\prime}\left|\Lambda_{1}^{3}(s)\right| s, m, i\right\rangle=\left[\lambda_{s}^{-} \operatorname{Tr}\left(\mathbb{1}_{m_{s}^{-}}\right)+\lambda_{s}^{+} \operatorname{Tr}\left(\mathbb{1}_{m_{s}^{+}}\right)\right] \delta_{s^{\prime} s} \delta_{m^{\prime} m}=\left(\lambda_{s}^{-} m_{s}^{-}+\lambda_{s}^{+} m_{s}^{+}\right) \delta_{s^{\prime} s} \delta_{m^{\prime} m}=\frac{d_{s}}{n} \delta_{s^{\prime} s} \delta_{m^{\prime} m}
$$

The representation (B120) implies that, $\forall s, m$,

$$
\sum_{i^{\prime}=1}^{d_{s^{\prime}}} \sum_{i=1}^{d_{s}}\left\langle s^{\prime}, m^{\prime}, i^{\prime}\left|\Lambda_{1}^{-}(s-1)\right| s, m, i\right\rangle=\lambda_{s}^{+} \operatorname{Tr}\left(\mathbb{1}_{m_{s}^{+}}\right) \delta_{s^{\prime} s-1} \delta_{m^{\prime} m}=\lambda_{s}^{+} m_{s}^{+} \delta_{s^{\prime} s-1} \delta_{m^{\prime} m}
$$

The representations (B119) implies that, $\forall s, m$,

$$
\sum_{i^{\prime}=1}^{d_{s^{\prime}}} \sum_{i=1}^{d_{s}}\left\langle s^{\prime}, m^{\prime}, i^{\prime}\left|\Lambda_{1}^{+}(s+1)\right| s, m, i\right\rangle=\lambda_{s+1}^{+} \operatorname{Tr}\left(\mathbb{1}_{m_{s}^{-}}\right) \delta_{s^{\prime} s+1} \delta_{m^{\prime} m}=\lambda_{s+1}^{+} m_{s}^{-} \delta_{s^{\prime} s+1} \delta_{m^{\prime} m}
$$

The matrix elements (B139) and (B140) satisfy the property (B39), as expected. The substitution of the matrix elements (B138), (B139), and (B140) into the matrix elements (B136) and (B137) yields the matrix elements (B134) and (B135).

[1] X. Xu, S. Yin, R. Moro, and W. A. de Heer, Phys. Rev. Lett. 95, 237209 (2005).

[2] W. A. de Heer, in Metal Clusters at Surfaces: Structure, Quantum Properties, Physical Chemistry, Chap. Confinement and Size Effects in Free Metal Clusters (Springer, Berlin, Heidelberg, 2000), pp. 1-35.

[3] W. A. de Heer, P. Milani, and A. Chatelain, Phys. Rev. Lett. 65, 488 (1990).

[4] A. Chatelain, Philos. Mag. Part B 79, 1367 (1999).

[5] F. W. Payne, W. Jiang, J. W. Emmert, J. Deng, and L. A. Bloomfield, Phys. Rev. B 75, 094431 (2007).

[6] W. Gerlach and O. Stern, Z. Phys. 9, 349 (1922).

[7] W. Gerlach and O. Stern, Z. Phys. 9, 353 (1922).

[8] T. E. Phipps and J. B. Taylor, Phys. Rev. 29, 309 (1927).

[9] P. J. Jensen and K. H. Bennemann, in New Trends in Magnetism, Magnetic Materials, and Their Applications, Chap. Magnetic Properties of Transition Metal and Rare-earth Metal Clusters (Springer, New York, 1994), pp. 21-28.

[10] W. A. de Heer and I. M. L. Billas, in New Trends in Magnetism, Magnetic Materials, and Their Applications, Chap. Magnetic
Properties of Small Transition Metal Clusters in a Molecular Beam (Springer, New York, 1994), pp. 9-19.

[11] S. Clough, J. Phys. C 9, 1553 (1976).

[12] J. Tejada, in Molecular Magnets: Physics and Applications, Chap. From Quantum Relaxation to Resonant Spin Tunneling (Springer, Berlin, Heidelberg, 2014), pp. 3-15.

[13] H. Breuer and F. Petruccione, The Theory of Open Quantum Systems (Oxford University Press, Oxford, UK, 2007).

[14] R. Alicki and M. Fannes, Quantum Dynamical Systems (Oxford University Press, Oxford, UK, 2001).

[15] J. von Neumann, Mathematical Foundation of Quantum Mechanics (Princeton University Press, Princeton, NJ, 1955).

[16] P. A. M. Dirac, Proc. Cambridge Philos. Soc. 25, 62 (1929).

[17] F. Bloch, Phys. Rev. 102, 104 (1956).

[18] R. K. Wangsness and F. Bloch, Phys. Rev. 89, 728 (1953).

[19] A. Redfield, in Advances in Magnetic Resonance, edited by J. S. Waugh, Vol. 1 of Advances in Magnetic and Optical Resonance (Academic Press, San Diego, 1965), pp. 1-32.

[20] G. Lindblad, Commun. Math. Phys. 40, 147 (1975). 
[21] S. H. Lin, J. Chem. Phys. 61, 3810 (1974).

[22] J. M. Jean, R. A. Friesner, and G. R. Fleming, J. Chem. Phys. 96, 5827 (1992).

[23] M. Tung and J.-M. Yuan, Phys. Rev. A 36, 4463 (1987).

[24] V. May and M. Schreiber, Phys. Rev. A 45, 2868 (1992).

[25] S. Gao, Phys. Rev. B 55, 1876 (1997).

[26] O. Linden and V. May, Eur. Phys. J. D 12, 473 (2000).

[27] F. Reuse, K. Maschke, V. de Coulon, J. van der Klink, and J.-P. Ansermet, Eur. Phys. J. B 36, 573 (2003).

[28] K. Blum, Density Matrix Theory and Applications (SpringerVerlag, Berlin, 2012).

[29] F. Reuse, V. de Coulon, and K. Maschke, J. Stat. Phys. 114, 361 (2004).

[30] S. D. Brechet, F. A. Reuse, K. Maschke, and J.-P. Ansermet, Eur. Phys. J. D 69, 180 (2015).

[31] S. Weinberg, Lectures on Quantum Mechanics (Cambridge University Press, Cambridge, UK, 2013).

[32] G. James and A. Kerber, The Representation Theory of the Symmetry Group (Addison-Wesley, Reading, MA, 1981).

[33] D. S. Passmann, Permutation Groups (W. A. Benjamin, Amsterdam, 1968).

[34] V. A. Yurovsky, Phys. Rev. Lett. 113, 200406 (2014).
[35] V. A. Yurovsky, Phys. Rev. A 93, 023613 (2016).

[36] W. C. Schieve and L. P. Horwitz, Quantum Statistical Mechanics (Cambridge University Press, Cambridge, UK, 2009).

[37] E. Wigner, Z. Phys. 43, 624 (1927).

[38] C. Eckart, Rev. Mod. Phys. 2, 305 (1930).

[39] E. Wigner, Group Theory and Its Application to the Quantum Mechanics of Atomic Spectra (Accademic Press, San Diego, 1959).

[40] L. C. Biedenharn and J. D. Louck, Angular Momentum in Quantum Physics: Theory and Application (Cambridge University Press, Cambridge, UK, 2009).

[41] V. A. Yurovsky, Phys. Rev. A 91, 053601 (2015).

[42] V. A. Yurovsky, Phys. Rev. A 92, 033618 (2015).

[43] C. Piron, Foundations of Quantum Physics (W. A. Benjamin, Amsterdam, 1976).

[44] D. Aerts, Found. Phys. 12, 1131 (1982).

[45] T. Wenckebach, Essentials of Dynamic Nuclear Polarization (Spindrift Publications, Falkirk, Scotland, 2016).

[46] J. Petersen, K. B. Møller, R. Rey, and J. T. Hynes, J. Phys. Chem. B 117, 4541 (2013).

[47] E. Torres, K. Kompa, F. Remacle, and R. Levine, Chem. Phys. 347, 531 (2008). 